\title{
Catalytic Asymmetric Transacetalization
}

\author{
Ilija Čorić, Sreekumar Vellalath, and Benjamin List*
}

Max-Planck-Institut für Kohlenforschung, Kaiser-Wilhelm-Platz 1, D-45470, Mülheim an der Ruhr, Germany

list@mpi-muelheim.mpg.de

\section{Supporting Information}

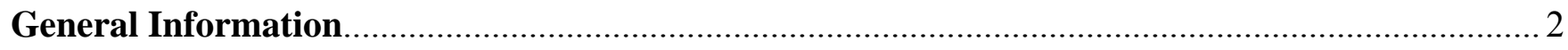

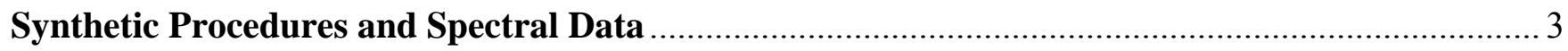

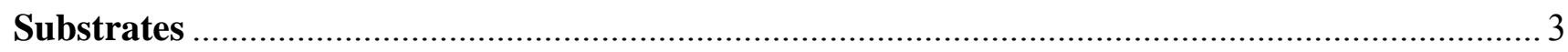

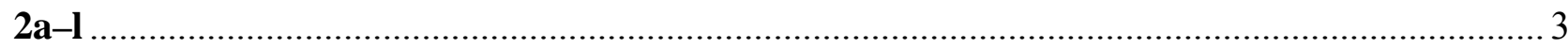

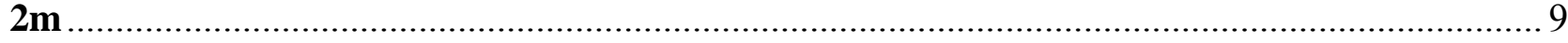

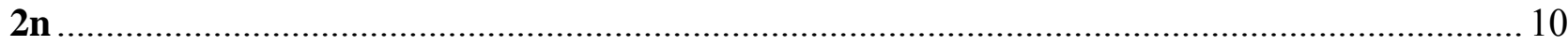

20

2p

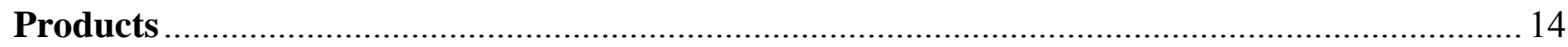

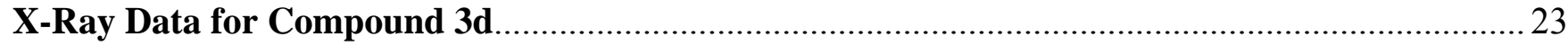

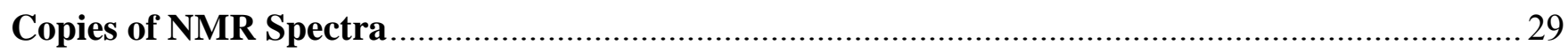

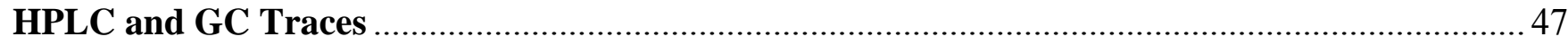




\section{General Information}

Unless otherwise stated, all reagents were purchased from commercial suppliers and used without further purification. All solvents used in the reactions were distilled from appropriate drying agents prior to use. Reactions were monitored by thin layer chromatography on silica gel pre-coated plastic sheets $(0.2 \mathrm{~mm}$, Machery-Nagel). Visualization was accomplished by irradiation with UV light at 254 $\mathrm{nm}$ and/or phosphomolybdic acid (PMA) stain. Column chromatography was performed on Merck silica gel (60, particle size 0.040-0.063 mm). Proton, and carbon NMR spectra were recorded on Bruker AV500 or Bruker AV-400 spectrometer in deuterated solvent. Proton chemical shifts are reported in ppm

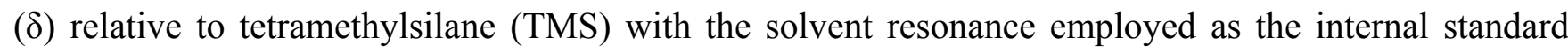
(DMSO, $\delta 2.49$ ppm; $\mathrm{CD}_{2} \mathrm{Cl}_{2}, \delta 5.32 \mathrm{ppm} ; \mathrm{CDCl}_{3} \delta$ 7.24). Data are reported as follows: chemical shift, multiplicity $(\mathrm{s}=$ singlet, $\mathrm{d}=$ doublet, $\mathrm{q}=$ quartet, $\mathrm{m}=$ multiplet $)$, coupling constants $(\mathrm{Hz})$ and integration. ${ }^{13} \mathrm{C}$ chemical shifts are reported in ppm from tetramethylsilane (TMS) with the solvent resonance as the internal standard $\left(\mathrm{DMSO}_{6}, \delta 39.5 \mathrm{ppm} ; \mathrm{CD}_{2} \mathrm{Cl}_{2}, 53.8 \mathrm{ppm} ; \mathrm{CDCl}_{3}, \delta\right.$ 77.0). High resolution mass spectra were determined on a Bruker APEX III FTMS (7 T magnet). The enantiomeric excesses were determined by GC or HPLC analysis employing a chiral stationary phase column specified in the individual experiment, by comparing the samples with the appropriate racemic mixtures. 


\section{Synthetic Procedures and Spectral Data}

\section{Substrates}

\section{$\underline{2 a-1}$}

\section{Representative procedure for compounds $2 a-1$}<smiles>CCOC(CCCl)OCC</smiles>

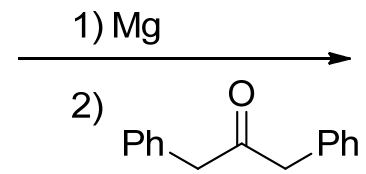<smiles>CCOC(CCC(O)(Cc1ccccc1)Cc1ccccc1)OCC</smiles>

2j

Preparation of the Grignard reagent

A solution of freshly distilled 3-chloropropionaldehyde diethylacetal $(5.0 \mathrm{~g}, 30.0 \mathrm{mmol})$ in THF (5 ml) was added to activated $^{i}$ magnesium turnings $(1.46 \mathrm{~g}, 60 \mathrm{mmol})$. The temperature of the exothermic reaction mixture was kept between $15-25{ }^{\circ} \mathrm{C}$ by cooling with an ice bath. ${ }^{i i,}$ iii After heat development ceased, the mixture was diluted with toluene $(10 \mathrm{ml})$ and the resulting solution (calculated ca. 1.5 $\mathrm{mmol} / \mathrm{ml}$ ) was used immediately.

\section{Addition to ketones}

A solution of the Grignard reagent prepared above $(7.5 \mathrm{ml}, 11.25 \mathrm{mmol})^{i v}$ was cooled to $-30{ }^{\circ} \mathrm{C}$ and a solution of ketone $(5.0 \mathrm{mmol})$ in toluene $(1.25 \mathrm{ml})$ was added dropwise. ${ }^{v}$ The mixture was allowed to warm to $0{ }^{\circ} \mathrm{C}$ during $2-4 \mathrm{~h}$. It was then quenched at $0{ }^{\circ} \mathrm{C}$ with concentrated aqueous $\mathrm{NH}_{4} \mathrm{Cl}(5 \mathrm{ml})$ and $\mathrm{H}_{2} \mathrm{O}(5 \mathrm{ml})$, and extracted with $\mathrm{Et}_{2} \mathrm{O}(2 \times 10 \mathrm{ml})$. The combined organic extracts were washed with conc. $\mathrm{Na}_{2} \mathrm{CO}_{3}$ (aq.) $(5 \mathrm{ml})$, dried $\left(\mathrm{MgSO}_{4}\right)$, filtered, and concentrated directly prior to purification. Flash chromatography on silica gel yielded pure products. ${ }^{v i}$ The products were stored as $0.1-0.2 \mathrm{M}$ solutions in $\mathrm{EtOAc}$ or $\mathrm{Et}_{2} \mathrm{O}$, or used immediately for transacetalization reaction. 
Notes:

- i) Activation was performed with a few drops of 1,2-dibromoethane.

- ii) For reactions on a larger scale a more efficient dry ice/acetone bath cooling is necessary.

- iii) If there is no exothermic reaction at this point, initiation is performed by adding more 1,2dibromoethane and fast heating (with a heat gun) and cooling to $25^{\circ} \mathrm{C}$ (repeated until reaction becomes exothermic after cooling).

- iv) For all other reactions, except $2 \mathbf{j}$ and $\mathbf{2 d}, 1.5$ equiv. of the Grignard reagent was used. However, incomplete conversion of the starting ketones was observed. This is probably a result of the decomposition of the Grignard reagent ${ }^{1}$ during preparation which leads to variable yields. Addition of more Grignard reagent (which was meanwhile kept at $-40{ }^{\circ} \mathrm{C}$ ) has beneficial effect on conversion.

- v) Ketones that are solids were added in portions to the Grignard reagent diluted with $1.25 \mathrm{ml}$ of toluene.

- vi) TLC visualization: UV (254 nm) and/or PMA stain.

${ }^{1}$ Greiner, A. Tetrahedron Lett. 1989, 30, 3547. 


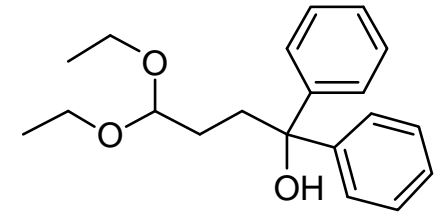

Purification: 10\% EtOAc/hexane. Colorless oil, $2.12 \mathrm{~g}$ (from $10.0 \mathrm{mmol}$ of ketone), 67\%. ${ }^{1} \mathrm{H}$ NMR (400 MHz, DMSO-d 6 ) $\delta 7.41(\mathrm{dd}, J=8.3,1.2 \mathrm{~Hz}$, 4H), $7.26(\mathrm{t}, J=7.8 \mathrm{~Hz}, 4 \mathrm{H}), 7.14(\mathrm{tt}, J=7.3,1.2 \mathrm{~Hz}, 2 \mathrm{H}), 5.50(\mathrm{~s}, 1 \mathrm{H}), 4.42$ $(\mathrm{t}, J=5.7 \mathrm{~Hz}, 1 \mathrm{H}) 3.51-3.43(\mathrm{~m}, 2 \mathrm{H}), 3.38-3.30(\mathrm{~m}, 2 \mathrm{H}), 2.24-2.20(\mathrm{~m}, 2 \mathrm{H}), 1.47-1.41(\mathrm{~m}, 2 \mathrm{H}), 1.06$ $(\mathrm{t}, J=7.1 \mathrm{~Hz}, 6 \mathrm{H}) .{ }^{13} \mathrm{C}$ NMR $\left(100 \mathrm{MHz}, \mathrm{DMSO}_{-} \mathrm{d}_{6}\right) \delta 148.2,127.7,126.0,125.7,102.4,76.1,60.2$, 36.0, 28.0, 15.3. HRMS (ESI+) $m / z$ calculated for $\mathrm{C}_{20} \mathrm{H}_{26} \mathrm{O}_{3} \mathrm{Na}\left(\mathrm{M}+\mathrm{Na}^{+}\right)$337.1774, found 337.1774.

\section{4,4-Diethoxy-1,1-bis(4-methoxyphenyl)butan-1-ol (2b)}

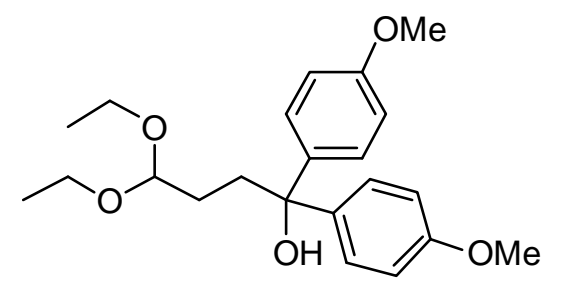

Purification: $20 \%$ EtOAc/hexane (mixture loaded on the column as a solution in toluene). Colorless solid, $776 \mathrm{mg}$ (from $5.0 \mathrm{mmol}$ of ketone), 41\%. ${ }^{1} \mathrm{H}$ NMR (400 MHz, DMSO-d 6 ) $\delta 7.27$ (d, $J=8.9 \mathrm{~Hz}$,

4H), $6.81(\mathrm{~d}, J=8.8 \mathrm{~Hz}, 4 \mathrm{H}), 5.30(\mathrm{~s}, 1 \mathrm{H}), 4.40(\mathrm{t}, J=5.6 \mathrm{~Hz}, 1 \mathrm{H}), 3.69$ (s, 6H), 3.51-3.43 (m, 2H), 3.38-3.30 (m, 2H), 2.16-2.12 (m, 2H), 1.45-1.40 (m, 2H), $1.06(\mathrm{t}, J=7.0 \mathrm{~Hz}, 6 \mathrm{H}) .{ }^{13} \mathrm{C}$ NMR $(100$ MHz, DMSO-d 6 ) $\delta 157.4,140.7,126.9,112.9,102.4,75.6,60.1,54.9,36.3,28.1,15.2$. HRMS (ESI+) $m / z$ calculated for $\mathrm{C}_{22} \mathrm{H}_{30} \mathrm{O}_{5} \mathrm{Na}\left(\mathrm{M}+\mathrm{Na}^{+}\right) 397.1985$, found 397.1982.

\section{4,4-Diethoxy-1,1-bis(4-fluorophenyl)butan-1-ol (2c)}

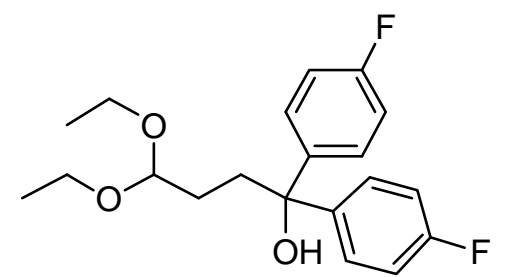

Purification: $20 \%$ EtOAc/hexane (mixture loaded on the column as a solution in toluene). Colorless oil, $781 \mathrm{mg}$ (from $5.0 \mathrm{mmol}$ of ketone), 45\%. ${ }^{1} \mathrm{H}$ NMR (400 MHz, DMSO-d 6 ) $\delta$ 7.43-7.38 (m, 4H), 7.11-7.05

(m, 4H), $5.64(\mathrm{~s}, 1 \mathrm{H}), 4.41(\mathrm{t}, J=5.6 \mathrm{~Hz}, 1 \mathrm{H}), 3.50-3.43(\mathrm{~m}, 2 \mathrm{H}), 3.38-3.30(\mathrm{~m}, 2 \mathrm{H}), 2.22-2.17(\mathrm{~m}$, 2H) $1.43-1.38(\mathrm{~m}, 2 \mathrm{H}), 1.06(\mathrm{t}, J=7.0 \mathrm{~Hz}, 6 \mathrm{H}) .{ }^{13} \mathrm{C}$ NMR $\left(100 \mathrm{MHz}, \mathrm{DMSO}-\mathrm{d}_{6}\right) \delta 160.6(\mathrm{~d}, J=242.3$ Hz), $144.3(\mathrm{~d}, J=3.0 \mathrm{~Hz}), 127.7$ (d, $J=7.9 \mathrm{~Hz}), 114.4(\mathrm{~d}, J=20.7 \mathrm{~Hz}), 102.3,75.6,60.2,36.1,28.0$, 15.3. HRMS (ESI+) $m / z$ calculated for $\mathrm{C}_{20} \mathrm{H}_{24} \mathrm{O}_{3} \mathrm{~F}_{2} \mathrm{Na}\left(\mathrm{M}+\mathrm{Na}^{+}\right)$373.1586, found 373.1588 . 


\section{1,1-Bis(4-bromophenyl)-4,4-diethoxybutan-1-ol (2d)}

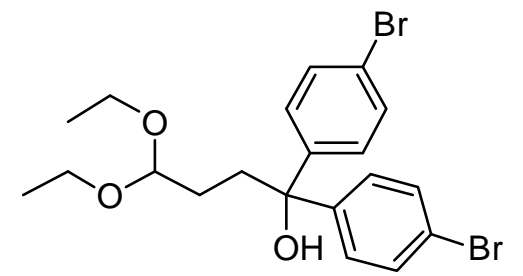

Purification: $\mathrm{CH}_{2} \mathrm{Cl}_{2} /$ hexane/EtOAc 10:10:1. Colorless oil, $823 \mathrm{mg}$ (from $3.15 \mathrm{mmol}$ of ketone), 55\%. ${ }^{1} \mathrm{H}$ NMR (500 MHz, DMSO-d 6 ) $\delta$ $7.46(\mathrm{~d}, J=8.6 \mathrm{~Hz}, 4 \mathrm{H}), 7.33(\mathrm{~d}, J=8.5 \mathrm{~Hz}, 4 \mathrm{H}), 5.74(\mathrm{~s}, 1 \mathrm{H}), 4.41$ (t, $J$ $=5.5 \mathrm{~Hz}, 1 \mathrm{H}), 3.50-3.43(\mathrm{~m}, 2 \mathrm{H}), 3.37-3.31(\mathrm{~m}, 2 \mathrm{H}), 2.20-2.17(\mathrm{~m}, 2 \mathrm{H}), 1.41-1.37(\mathrm{~m}, 2 \mathrm{H}), 1.06(\mathrm{t}, J$ $=7.0 \mathrm{~Hz}, 6 \mathrm{H}) .{ }^{13} \mathrm{C}$ NMR $\left(125 \mathrm{MHz}, \mathrm{DMSO}-\mathrm{d}_{6}\right) \delta 147.2,130.8,128.1,119.5,102.2,75.7,60.3,35.5$, 27.9, 15.3. HRMS (ESI+) $\mathrm{m} / z$ calculated for $\mathrm{C}_{20} \mathrm{H}_{24} \mathrm{O}_{3} \mathrm{Br}_{2} \mathrm{Na}\left(\mathrm{M}+\mathrm{Na}^{+}\right)$492.9985, found 492.9989 .

\section{9-(3,3-Diethoxypropyl)-9,10-dihydroanthracen-9-ol (2e)}

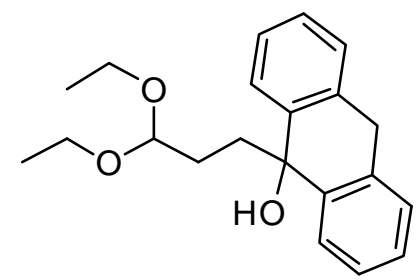

Purification: 15\% EtOAc/hexane (crude product dissolved in $10 \%$ EtOAc/hexane $(5 \mathrm{ml})$, filtered and concentrated prior to chromatography).

Colorless solid, $533 \mathrm{mg}$ (from $5.0 \mathrm{mmol}$ of ketone), 33\%. ${ }^{1} \mathrm{H}$ NMR (400 MHz, DMSO-d 6 ) $\delta 7.69(\mathrm{dd}, J=7.6,1.0 \mathrm{~Hz}, 2 \mathrm{H}), 7.31-7.26(\mathrm{~m}, 4 \mathrm{H}), 7.21(\mathrm{td}, J=7.3,1.2 \mathrm{~Hz}, 2 \mathrm{H})$, $5.72(\mathrm{~s}, 1 \mathrm{H}), 4.17(\mathrm{t}, J=5.7 \mathrm{~Hz}, 1 \mathrm{H}), 3.94(\mathrm{~s}, 2 \mathrm{H}), 3.39-3.31(\mathrm{~m}, 2 \mathrm{H}), 3.26-3.18(\mathrm{~m}, 2 \mathrm{H}), 1.57-1.53(\mathrm{~m}$, $2 \mathrm{H}), 1.38-1.32(\mathrm{~m}, 2 \mathrm{H}), 0.98(\mathrm{t}, J=7.0 \mathrm{~Hz}, 6 \mathrm{H}) .{ }^{13} \mathrm{C}$ NMR $\left(100 \mathrm{MHz}, \mathrm{DMSO}-\mathrm{d}_{6}\right) \delta 143.8,133.7$, 127.0, 126.3, 125.9, 125.1, 102.0, 72.6, 60.2, 37.6, 34.3, 28.1, 15.2. HRMS (ESI+) $m / z$ calculated for $\mathrm{C}_{21} \mathrm{H}_{26} \mathrm{O}_{3} \mathrm{Na}\left(\mathrm{M}+\mathrm{Na}^{+}\right)$349.1774, found 349.1776.

\section{5,5-Diethoxy-2-methylpentan-2-ol (2f)}

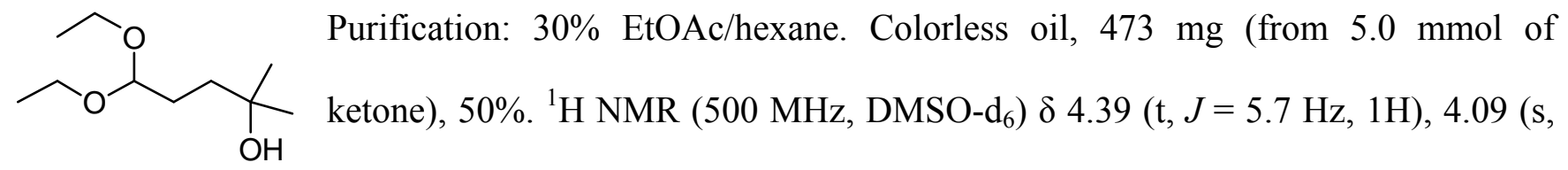
1H), 3.56-3.50 (m, 2H), 3.42-3.36 (m, 2H), 1.55-1.50 (m, 2H), 1.34-1.31 (m, 2H), 1.09 (t, $J=7.0 \mathrm{~Hz}$, $6 \mathrm{H}), 1.04(\mathrm{~s}, 6 \mathrm{H}) .{ }^{13} \mathrm{C}$ NMR (125 MHz, DMSO-d 6 ) $\delta 102.7,68.3,60.2,38.3,29.2,28.3,15.2 . \mathrm{HRMS}$ (ESI+) $m / z$ calculated for $\mathrm{C}_{10} \mathrm{H}_{22} \mathrm{O}_{3} \mathrm{Na}\left(\mathrm{M}+\mathrm{Na}^{+}\right)$213.1461, found 213.1461. 


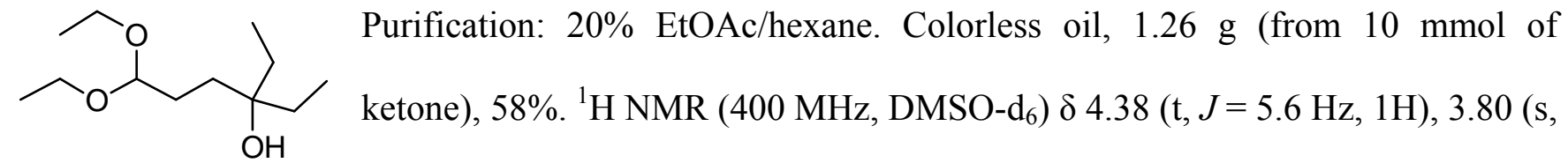
$1 \mathrm{H}), 3.56-3.49(\mathrm{~m}, 2 \mathrm{H}), 3.43-3.35(\mathrm{~m}, 2 \mathrm{H}), 1.48-1.42(\mathrm{~m}, 2 \mathrm{H}), 1.32-1.26(\mathrm{~m}, 6 \mathrm{H}), 1.08(\mathrm{t}, J=7.0 \mathrm{~Hz}$ $6 \mathrm{H}), 0.74(\mathrm{t}, J=7.5 \mathrm{~Hz}, 6 \mathrm{H}) .{ }^{13} \mathrm{C}$ NMR $\left(100 \mathrm{MHz}, \mathrm{DMSO}-\mathrm{d}_{6}\right) \delta 102.8,72.1,60.3,32.5,30.5,27.5$, 15.3, 7.8. HRMS (CI (FE) $i$-butane) $m / z$ calculated for $\mathrm{C}_{12} \mathrm{H}_{27} \mathrm{O}_{3}(\mathrm{M}+\mathrm{H}) 219.1960$, found 219.1962.

\section{6,6-Diethoxy-3-isopropyl-2-methylhexan-3-ol (2h)}

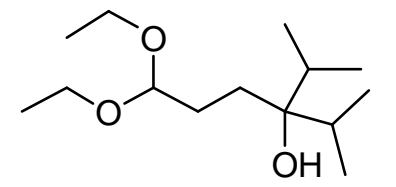

Purification: 15\% EtOAc/hexane. Colorless oil, $645 \mathrm{mg}$ (from $5.0 \mathrm{mmol}$ of ketone), 52\%. ${ }^{1} \mathrm{H}$ NMR (500 MHz, DMSO-d 6 ) $\delta 4.35$ (t, $\left.J=5.4 \mathrm{~Hz}, 1 \mathrm{H}\right), 3.56-$ $3.50(\mathrm{~m}, 2 \mathrm{H}), 3.52(\mathrm{~s}, 1 \mathrm{H}), 3.42-3.36(\mathrm{~m}, 2 \mathrm{H}), 1.81-1.72(\mathrm{~m}, 2 \mathrm{H}), 1.51-1.46(\mathrm{~m}, 2 \mathrm{H}), 1.39-1.36(\mathrm{~m}$, 2H), $1.09(\mathrm{t}, J=7.0 \mathrm{~Hz}, 6 \mathrm{H}), 0.85(\mathrm{~d}, J=6.9 \mathrm{~Hz}, 6 \mathrm{H}), 0.83(\mathrm{~d}, J=7.0 \mathrm{~Hz}, 6 \mathrm{H}) .{ }^{13} \mathrm{C} \mathrm{NMR}(125 \mathrm{MHz}$, DMSO-d $\left._{6}\right) \delta 103.0,75.0,60.4,33.4,28.4,28.1,17.6,17.3,15.3$. HRMS (ESI +$) \mathrm{m} / z$ calculated for $\mathrm{C}_{14} \mathrm{H}_{30} \mathrm{O}_{3} \mathrm{Na}\left(\mathrm{M}+\mathrm{Na}^{+}\right)$269.2087, found 269.2084.

\section{3-(tert-Butyl)-6,6-diethoxy-2,2-dimethylhexan-3-ol (2i)}

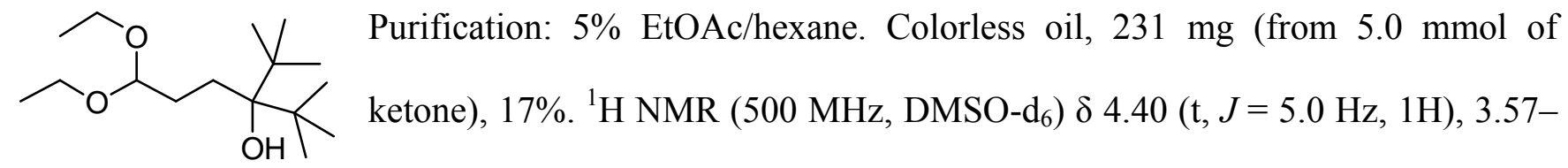
$3.50(\mathrm{~m}, 2 \mathrm{H}), 3.42-3.36(\mathrm{~m}, 2 \mathrm{H}), 3.35(\mathrm{~s}, 1 \mathrm{H}), 1.61-1.52(\mathrm{~m}, 4 \mathrm{H}), 1.09(\mathrm{t}, J=7.1 \mathrm{~Hz}, 6 \mathrm{H}), 0.97(\mathrm{~s}$, 18H). ${ }^{13} \mathrm{C}$ NMR (125 MHz, DMSO-d 6 ) $\delta 103.3,78.1,60.4,42.2,30.4,28.6,27.4,15.3$. HRMS (ESI+) $m / z$ calculated for $\mathrm{C}_{16} \mathrm{H}_{34} \mathrm{O}_{3} \mathrm{Na}\left(\mathrm{M}+\mathrm{Na}^{+}\right)$297.2400, found 297.2398. 


\section{2-Benzyl-5,5-diethoxy-1-phenylpentan-2-ol (2j)}

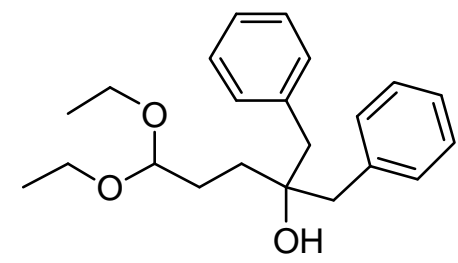

Purification: 15\% EtOAc/hexane. Colorless oil, $1.009 \mathrm{~g}$ (from $5.0 \mathrm{mmol}$ of ketone), 59\%. ${ }^{1} \mathrm{H}$ NMR $(500 \mathrm{MHz}$, DMSO-d 6 ) $\delta 7.24(\mathrm{~d}, J=4.4 \mathrm{~Hz}, 8 \mathrm{H})$, 7.19-7.15 (m, 2H), $4.29(\mathrm{~s}, 1 \mathrm{H}), 4.23(\mathrm{t}, J=5.6 \mathrm{~Hz}, 1 \mathrm{H}), 3.47-3.41(\mathrm{~m}$, 2H), 3.35-3.28 (m, 2H), 2.67 (d, $J=13.6 \mathrm{~Hz}, 2 \mathrm{H}), 2.63(\mathrm{~d}, J=13.6 \mathrm{~Hz}, 2 \mathrm{H}), 1.64-1.60(\mathrm{~m}, 2 \mathrm{H}), 1.20$ $1.17(\mathrm{~m}, 2 \mathrm{H}), 1.02(\mathrm{t}, J=7.0 \mathrm{~Hz}, 6 \mathrm{H}) .{ }^{13} \mathrm{C}$ NMR $\left(125 \mathrm{MHz}, \mathrm{DMSO}-\mathrm{d}_{6}\right) \delta 138.3,130.6,127.5,125.8$, 102.8, 73.3, 60.4, 45.3, 32.3, 28.0, 15.2. HRMS $(\mathrm{ESI}+) \mathrm{m} / \mathrm{z}$ calculated for $\mathrm{C}_{22} \mathrm{H}_{30} \mathrm{O}_{3} \mathrm{Na}\left(\mathrm{M}+\mathrm{Na}^{+}\right)$ 365.2087, found 365.2086 .

\section{1-(3,3-Diethoxypropyl)cyclohexanol (2k)}

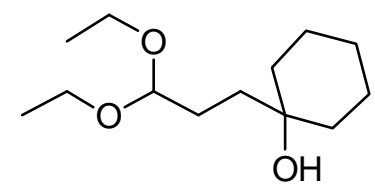

Purification: 25\% EtOAc/hexane. Colorless oil, $656 \mathrm{mg}$ (from $5.0 \mathrm{mmol}$ of ketone), 57\%. ${ }^{1} \mathrm{H}$ NMR (500 MHz, DMSO-d 6 ) $\delta 4.38(\mathrm{t}, J=5.6 \mathrm{~Hz}, 1 \mathrm{H}), 3.83$ (s, 1H), 3.55-3.49 (m, 2H), 3.42-3.36 (m, 2H), 1.60-1.49 (m, 4H), 1.45-1.37 (m, 3H), 1.33-1.29 (m, 4H), $1.25-1.16(\mathrm{~m}, 3 \mathrm{H}), 1.09$ (t, $J=7.0 \mathrm{~Hz}, 6 \mathrm{H}) .{ }^{13} \mathrm{C}$ NMR (125 MHz, DMSO-d 6 ) $\delta 102.8,68.8,60.1,37.0$, 36.9, 27.0, 25.7, 21.8, 15.3. HRMS (ESI+) $m / z$ calculated for $\mathrm{C}_{13} \mathrm{H}_{26} \mathrm{O}_{3} \mathrm{Na}\left(\mathrm{M}+\mathrm{Na}^{+}\right) 253.1774$, found 253.1772 .

\section{1-(3,3-Diethoxypropyl)-2,3-dihydro-1H-inden-1-ol (2l)}

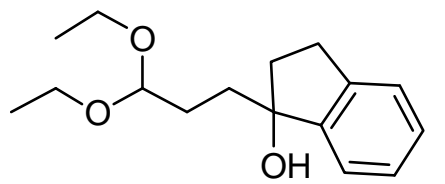

Purification: 25\% EtOAc/hexane. Colorless oil, $684 \mathrm{mg}$ (from $5.0 \mathrm{mmol}$ of

ketone), 52\%. ${ }^{1} \mathrm{H}$ NMR (500 MHz, DMSO-d 6 ) $\delta 7.26-7.23(\mathrm{~m}, 1 \mathrm{H}), 7.19$ 7.17 (m, 3H), $4.92(\mathrm{~s}, 1 \mathrm{H}), 4.40$ (t, $J=5.2 \mathrm{~Hz}, 1 \mathrm{H}), 3.54-3.47$ (m, 2H), 3.40-3.34 (m, 2H), 2.89-2.84 (m, 1H), 2.73-2.67 (m, 1H), 2.12-2.07 (m, 1H), 2.00-1.94 (m, 1H), 1.74-1.70 (m, 1H), 1.64-1.55 (m, 2H), $1.53-1.47(\mathrm{~m}, 1 \mathrm{H}), 1.073(\mathrm{t}, J=7.0 \mathrm{~Hz}, 3 \mathrm{H}), 1.068(\mathrm{t}, J=7.0 \mathrm{~Hz}, 3 \mathrm{H}) .{ }^{13} \mathrm{C}$ NMR $(125 \mathrm{MHz}$, DMSO-d $\left.{ }_{6}\right) \delta 148.7,142.3,127.3,126.1,124.5,123.1,102.5,81.5,60.5,60.2,39.4,35.4,29.0,28.4$, 15.3. HRMS (ESI+) $m / z$ calculated for $\mathrm{C}_{16} \mathrm{H}_{24} \mathrm{O}_{3} \mathrm{Na}\left(\mathrm{M}+\mathrm{Na}^{+}\right)$287.1618, found 287.1616. 


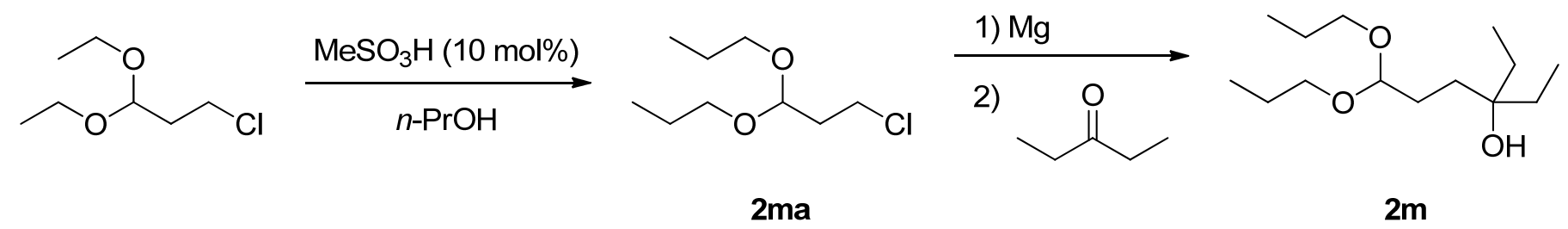

\section{3-Chloro-1,1-diethoxypropane (2ma)}

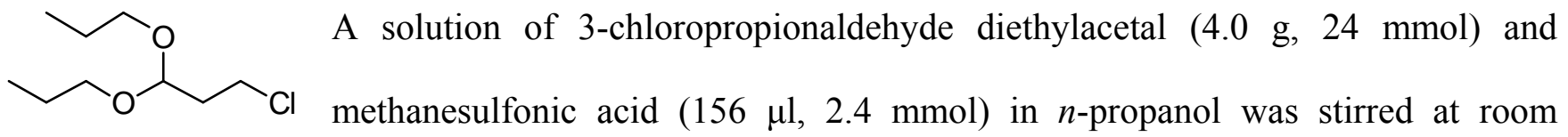
temperature for $16 \mathrm{~h}$. The volume of the mixture was slowly reduced to ca. $10 \mathrm{ml}$ under reduced pressure at $42{ }^{\circ} \mathrm{C}$. Sodium hydrogencarbonate $(403 \mathrm{mg}, 4.8 \mathrm{mmol}$ ) was then added and the remaining $n$ propanol removed under reduced pressure. $\mathrm{Et}_{2} \mathrm{O}(20 \mathrm{ml})$ was added and the resulting solution washed with $\mathrm{H}_{2} \mathrm{O}(3 \times 10 \mathrm{ml})$, conc. aqueous $\mathrm{Na}_{2} \mathrm{CO}_{3}(5 \mathrm{ml})$, dried $\left(\mathrm{MgSO}_{4}\right)$, and the solvent was removed under reduced pressure. Purified by Kughelrohr distillation $\left(80^{\circ} \mathrm{C}\right.$, reduced pressure). Colorless oil, $3.80 \mathrm{~g}, 81 \% .{ }^{1} \mathrm{H}$ NMR (400 MHz, $\left.\mathrm{CDCl}_{3}\right) \delta 4.66(\mathrm{t}, J=5.6 \mathrm{~Hz}, 1 \mathrm{H}), 3.60-3.53$ (m, $\left.4 \mathrm{H}\right), 3.42-3.37$ (m, 2H), $2.05(\mathrm{td}, J=6.6,5.7 \mathrm{~Hz}, 2 \mathrm{H}), 1.63-1.54(\mathrm{~m}, 4 \mathrm{H}), 0.92(\mathrm{t}, J=7.4 \mathrm{~Hz}, 6 \mathrm{H}) .{ }^{13} \mathrm{C}$ NMR $(100 \mathrm{MHz}$, $\left.\mathrm{CDCl}_{3}\right) \delta 100.7,68.4,40.9,36.8,23.1,10.7$.

\section{3-Ethyl-6,6-dipropoxyhexan-3-ol (2m)}

Representative procedure for compounds 2a-l was followed using 3-chloro-
1,1-diethoxypropane (2ma) and 3-pentanone as substrates. Purification: $15 \%$ EtOAc/hexane. Colorless oil, $1.08 \mathrm{~g}$ (from $8.125 \mathrm{mmol}$ of ketone), 54\%. ${ }^{1} \mathrm{H}$ NMR (500 MHz, DMSO$\left.\mathrm{d}_{6}\right) \delta 4.38(\mathrm{t}, J=5.6 \mathrm{~Hz}, 1 \mathrm{H}), 3.79(\mathrm{~s}, 1 \mathrm{H}), 3.44(\mathrm{dt}, J=9.3,6.6 \mathrm{~Hz}, 2 \mathrm{H}), 3.30(\mathrm{dt}, J=9.3,6.6 \mathrm{~Hz}, 2 \mathrm{H})$, $1.52-1.45(\mathrm{~m}, 6 \mathrm{H}), 1.32-1.27(\mathrm{~m}, 6 \mathrm{H}), 0.86(\mathrm{t}, J=7.5 \mathrm{~Hz}, 6 \mathrm{H}), 0.75(\mathrm{t}, J=7.5 \mathrm{~Hz}, 6 \mathrm{H}) .{ }^{13} \mathrm{C}$ NMR $(125$ MHz, DMSO-d $\left.\mathrm{d}_{6}\right) \delta 103.0,72.1,66.4,32.5,30.5,27.4,22.7,10.7,7.8$. HRMS (ESI + ) $\mathrm{m} / z$ calculated for $\mathrm{C}_{14} \mathrm{H}_{30} \mathrm{O}_{3} \mathrm{Na}\left(\mathrm{M}+\mathrm{Na}^{+}\right)$269.2087, found 269.2084. 


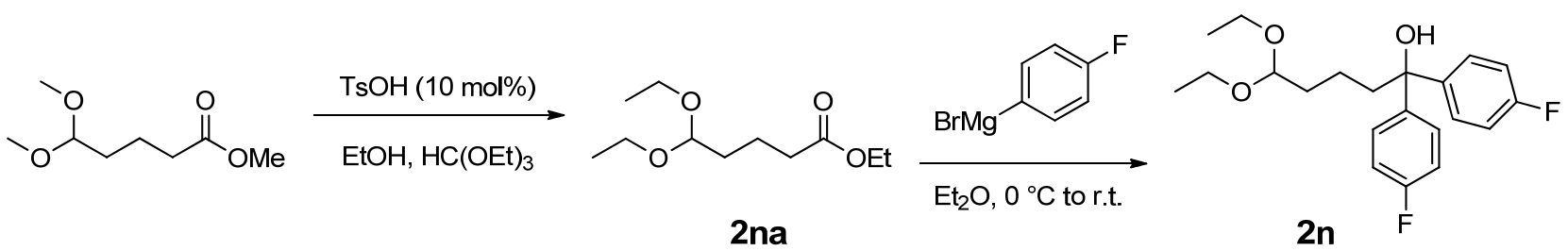

Ethyl 5,5-diethoxypentanoate (2na)<smiles>CCOC(=O)CCCC(OCC)OCC</smiles>

A solution of methyl 5,5-dimethoxypentanoate (1.76 g, $10 \mathrm{mmol})$, triethyl orthoformate $(2 \mathrm{ml})$, and p-toluenesulfonic acid monohydrate $(172 \mathrm{mg}, 1$ mmol) in ethanol $(25 \mathrm{ml})$ was heated at reflux. The solvent was slowly distilled off to ca. $10 \mathrm{ml}$ during 8 h. More ethanol $(10 \mathrm{ml})$ was added and further $10 \mathrm{ml}$ of solvent distilled off from the reaction mixture during $6 \mathrm{~h}$. The reaction mixture was cooled to room temperature, sodium carbonate (106 $\mathrm{mg}, 1 \mathrm{mmol})$ added and solvent removed under reduced pressure. Diethyl ether $(20 \mathrm{ml})$ was added to the residue, and the solution was washed with $0.5 \mathrm{M}$ aqueous $\mathrm{Na}_{2} \mathrm{CO}_{3}$ solution $(2 \times 10 \mathrm{ml})$, water $(3 \times 10 \mathrm{ml})$, brine $(10$ $\mathrm{ml})$, dried $\left(\mathrm{MgSO}_{4}\right)$, and concentrated under reduced pressure. Yellowish liquid, $2.17 \mathrm{~g}, 99 \%$. ${ }^{1} \mathrm{H}$ NMR $\left(500 \mathrm{MHz}, \mathrm{DMSO}-\mathrm{d}_{6}\right) \delta 4.43(\mathrm{t}, J=4.9 \mathrm{~Hz}, 1 \mathrm{H}), 4.03(\mathrm{q}, J=7.1 \mathrm{~Hz}, 2 \mathrm{H}), 3.57-3.50(\mathrm{~m}, 2 \mathrm{H}), 3.42-3.36$ $(\mathrm{m}, 2 \mathrm{H}), 2.28(\mathrm{t}, J=6.8 \mathrm{~Hz}, 2 \mathrm{H}), 1.55-1.47(\mathrm{~m}, 4 \mathrm{H}), 1.16(\mathrm{t}, J=7.1 \mathrm{~Hz}, 3 \mathrm{H}), 1.08(\mathrm{t}, J=7.1 \mathrm{~Hz}, 6 \mathrm{H})$. ${ }^{13} \mathrm{C}$ NMR $\left(125 \mathrm{MHz}, \mathrm{DMSO}-\mathrm{d}_{6}\right) \delta 172.8,101.8,60.4,59.6,33.1,32.4,19.8,15.3,14.1 . \mathrm{HRMS}(\mathrm{ESI}+)$ $m / z$ calculated for $\mathrm{C}_{11} \mathrm{H}_{22} \mathrm{O}_{4} \mathrm{Na}\left(\mathrm{M}+\mathrm{Na}^{+}\right)$241.1410, found 241.1409.

\section{5,5-Diethoxy-1,1-bis(4-fluorophenyl)pentan-1-ol (2n)}<smiles>CCOC(CCCC(O)(c1ccc(F)cc1)c1ccc(F)cc1)OCC</smiles>

room temperature, then cooled to $0^{\circ} \mathrm{C}$ and quenched with a concentrated ammonium chloride solution 
$(5 \mathrm{ml})$. Diethyl ether $(10 \mathrm{ml})$ and water $(5 \mathrm{ml})$ were added to the mixture. The organic layer was separated, washed with concentrated sodium carbonate solution $(5 \mathrm{ml})$, dried $\left(\mathrm{MgSO}_{4}\right)$, and concentrated under reduced pressure. Purification by silica gel chromatography (15\% EtOAc/hexane) yielded a colorless oil, $541 \mathrm{mg}, 74 \% .{ }^{1} \mathrm{H}$ NMR (500 MHz, DMSO-d 6 ) $\delta 7.44-7.40$ (m, 4H), 7.09-7.04 $(\mathrm{m}, 4 \mathrm{H}), 5.59(\mathrm{~s}, 1 \mathrm{H}), 4.37(\mathrm{t}, J=5.6 \mathrm{~Hz}, 1 \mathrm{H}), 3.51-3.45(\mathrm{~m}, 2 \mathrm{H}), 3.39-3.31(\mathrm{~m}, 2 \mathrm{H}), 2.21-2.17(\mathrm{~m}$, 2H), 1.50-1.46 (m, 2H), 1.23-1.16 (m, 2H), $1.03(\mathrm{t}, J=7.0 \mathrm{~Hz}, 6 \mathrm{H}) .{ }^{13} \mathrm{C}$ NMR $\left(125 \mathrm{MHz}, \mathrm{DMSO}-\mathrm{d}_{6}\right) \delta$ $160.6(\mathrm{~d}, J=242.5 \mathrm{~Hz}), 144.5(\mathrm{~d}, J=2.5 \mathrm{~Hz}), 127.7(\mathrm{~d}, J=7.8 \mathrm{~Hz}), 114.4(\mathrm{~d}, J=20.9 \mathrm{~Hz}), 102.1,75.9$, 60.4, 41.0, 33.5, 18.7, 15.3. HRMS (EI (DE)) $\mathrm{m} / z$ calculated for $\mathrm{C}_{21} \mathrm{H}_{26} \mathrm{O}_{3} \mathrm{~F}_{2}$ (M) 364.1850, found 364.1849.

\section{$\underline{20}$}<smiles>O=C(O)C1CCCCC1</smiles>

a) LDA, THF, $0^{\circ} \mathrm{C}$ to r.t.
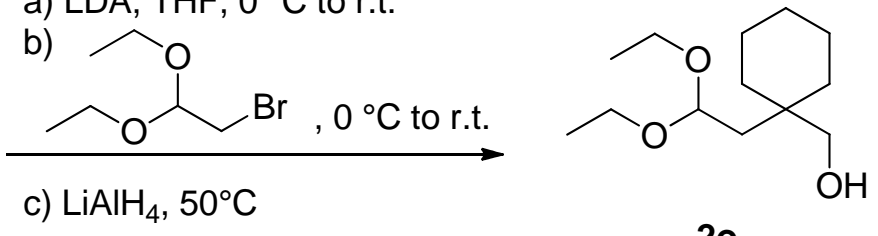

20

\section{(1-(2,2-Diethoxyethyl)cyclohexyl)methanol (2o)}

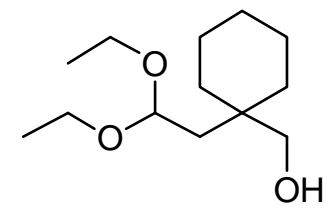

a) A $2.5 \mathrm{M}$ solution of $n$-BuLi $(11.8 \mathrm{ml}, 29.4 \mathrm{mmol})$ in hexanes was added dropwise to a solution of diisopropyl amine $(4.53 \mathrm{ml}, 32.3 \mathrm{mmol})$ in dry THF (28 $\mathrm{ml}$ ) at $0{ }^{\circ} \mathrm{C}$ and stirred for $30 \mathrm{~min}$ at $0{ }^{\circ} \mathrm{C}$. Then a solution of cyclohexanecarboxylic acid $(1.79 \mathrm{~g}, 14 \mathrm{mmol})$ in THF $(3 \mathrm{ml})$ was added and the mixture stirred for 30 min at $0{ }^{\circ} \mathrm{C}$ and $2 \mathrm{~h}$ at room temperature. b) The mixture was cooled to $0{ }^{\circ} \mathrm{C}$ and bromoacetaldehyde diethyl acetal $(2.32 \mathrm{ml}, 3.03 \mathrm{~g}, 15.4 \mathrm{mmol})$ was added dropwise. The mixture was stirred for $30 \mathrm{~min}$ at 0 ${ }^{\circ} \mathrm{C}$ and $12 \mathrm{~h}$ at room temperature. c) Lithium aluminum hydride (1.06 g, $\left.28 \mathrm{mmol}\right)$ was added in small portions and the reaction mixture stirred at $50{ }^{\circ} \mathrm{C}$ for $2.5 \mathrm{~h}$. The mixture was cooled to room temperature and poured slowly on the mixture of water $(50 \mathrm{ml})$ and diethyl ether $(150 \mathrm{ml})$. The organic layer was separated by decantation, and the aqueous slurry extracted with diethyl ether $(6 \times 25 \mathrm{ml})$. The combined 
organic extracts were washed with concentrated aqueous sodium carbonate solution $(2 \times 25 \mathrm{ml})$, dried $\left(\mathrm{MgSO}_{4}\right)$, and concentrated directly prior to purification. Purification by silica gel chromatography (hexane/EtOAc/MeOH 9:1:0.2, $\left.\mathrm{R}_{\mathrm{f}}=0.31\right)$ yielded a colorless oil, $1.31 \mathrm{~g}, 41 \% .{ }^{1} \mathrm{H} \mathrm{NMR}(500 \mathrm{MHz}$, DMSO-d $)_{6} \delta 4.56(\mathrm{t}, J=5.3 \mathrm{~Hz}, 1 \mathrm{H}), 4.26(\mathrm{t}, J=5.3 \mathrm{~Hz}, 1 \mathrm{H}), 3.56-3.50(\mathrm{~m}, 2 \mathrm{H}), 3.42-3.36(\mathrm{~m}, 2 \mathrm{H})$, $3.23(\mathrm{~d}, J=5.4 \mathrm{~Hz}, 2 \mathrm{H}), 1.51(\mathrm{~d}, J=5.3 \mathrm{~Hz}, 2 \mathrm{H}), 1.39-1.34(\mathrm{~m}, 5 \mathrm{H}), 1.29-1.22(\mathrm{~m}, 5 \mathrm{H}), 1.08(\mathrm{t}, J=7.0$ $\mathrm{Hz}, 6 \mathrm{H}) .{ }^{13} \mathrm{C}$ NMR $\left(125 \mathrm{MHz}, \mathrm{DMSO}-\mathrm{d}_{6}\right) \delta 100.3,66.8,60.2,38.2,36.0,32.5,26.0,21.2,15.3 . \mathrm{HRMS}$ (ESI+) $m / z$ calculated for $\mathrm{C}_{13} \mathrm{H}_{26} \mathrm{O}_{3} \mathrm{Na}\left(\mathrm{M}+\mathrm{Na}^{+}\right)$253.1774, found 253.1774. 


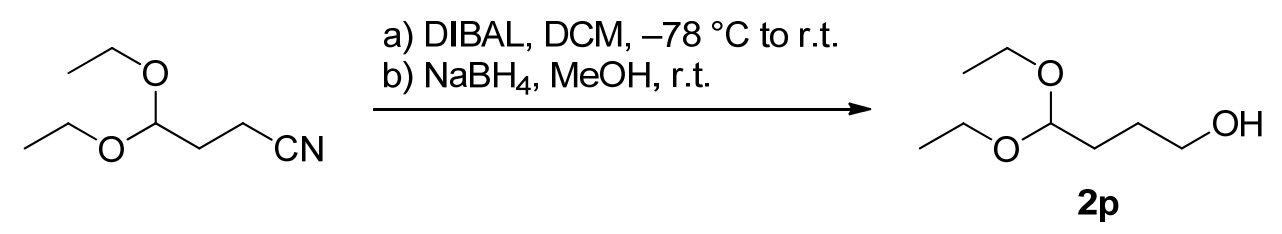

\section{4,4-Diethoxybutan-1-ol (2p)}

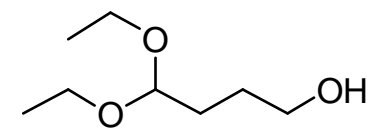

a) A 1.0 M solution of DIBAL (6 ml, $6.0 \mathrm{mmol})$ in toluene was added to a solution of 3-cyanopropionaldehyde diethyl acetal $(943 \mathrm{mg}, 6.0 \mathrm{mmol})$ in dry DCM $(30 \mathrm{ml})$ at $-78{ }^{\circ} \mathrm{C}$ and stirred for $1.5 \mathrm{~h}$ at $-78^{\circ} \mathrm{C}$. The mixture was allowed to warm to room temperature, treated with concentrated aqueous ammonium chloride solution $(10 \mathrm{ml})$ and water $(10 \mathrm{ml})$, and extracted with DCM $(3 \times 20 \mathrm{ml})$. The combined organic extracts were washed with brine $(2 \times 20$ $\mathrm{ml})$, dried $\left(\mathrm{MgSO}_{4}\right)$, and concentrated. b) The oily residue was dissolved in methanol (10 ml), $\mathrm{NaBH}_{4}$ (227 mg, $6 \mathrm{mmol}$ ) was added and the mixture was stirred at room temperature. After $1 \mathrm{~h}$ water $(10 \mathrm{ml})$ was added to the mixture, methanol was removed under reduced pressure, and the resulting mixture was extracted with ethyl acetate $(3 \times 20 \mathrm{ml})$. The combined organic extracts were washed with concentrated aqueous sodium carbonate solution $(10 \mathrm{ml})$, dried $\left(\mathrm{MgSO}_{4}\right)$, and concentrated. Purification by silica gel chromatography (50\% EtOAc/hexane) yielded a colorless oil, $407 \mathrm{mg}, 42 \% .{ }^{1} \mathrm{H}$ NMR (500 MHz, DMSO-d $\left._{6}\right) \delta 4.43(\mathrm{t}, J=5.6 \mathrm{~Hz}, 1 \mathrm{H}), 4.37(\mathrm{t}, J=5.2 \mathrm{~Hz}, 1 \mathrm{H}), 3.56-3.50(\mathrm{~m}, 2 \mathrm{H}), 3.43-3.35(\mathrm{~m}, 4 \mathrm{H})$, $1.53-1.48(\mathrm{~m}, 2 \mathrm{H}), 1.43-1.38(\mathrm{~m}, 2 \mathrm{H}), 1.09(\mathrm{t}, J=7.1 \mathrm{~Hz}, 6 \mathrm{H}) .{ }^{13} \mathrm{C}$ NMR (125 MHz, DMSO-d 6 ) $\delta$ 102.1, 60.5, 60.3, 29.9, 27.8, 15.3. HRMS (CI (FE) $i$-butane) $\mathrm{m} / z$ calculated for $\mathrm{C}_{8} \mathrm{H}_{19} \mathrm{O}_{3}(\mathrm{M}+\mathrm{H})$ 163.1334, found 163.1332 . 


\section{Products}

\section{General procedure for catalytic enantioselective intramolecular transacetalization}<smiles>[R]C([R])(O)CCC(OCC)OCC</smiles>

2

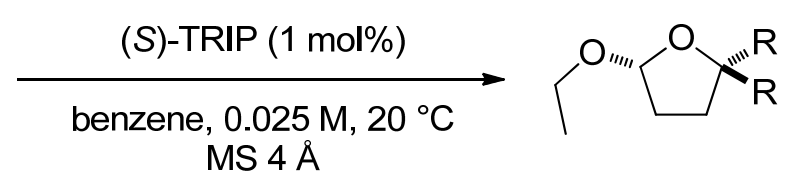

3

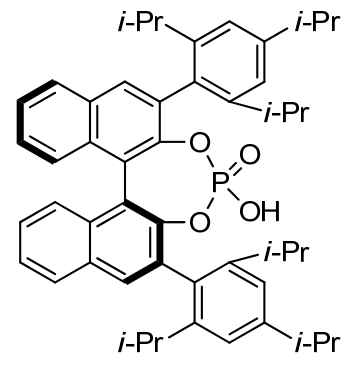

(S)-TRIP (1a)

Molecular sieves $4 \AA(150 \mathrm{mg})$ and a solution of $(S)$-TRIP $(2.38 \mathrm{mg}, 0.003 \mathrm{mmol}$, co-crystallized with $\mathrm{CH}_{3} \mathrm{CN} \mathrm{1:1)}$ in dry benzene $(4 \mathrm{ml})$, were added to the solution of $2(0.3 \mathrm{mmol})$ in dry benzene $(8 \mathrm{ml})$ and stirred at $20^{\circ} \mathrm{C}$. $\mathrm{Et}_{3} \mathrm{~N}(42 \mu \mathrm{l})$ was added to the reaction mixture after complete conversion of the starting material (in most cases within $9-24 \mathrm{~h}){ }^{i}$

- For non-volatile products: $:^{i i}$ The reaction mixture was concentrated under reduced pressure and the product was purified by silica gel ( $5 \mathrm{~g})$ chromatography with EtOAc/hexane as the eluent. ${ }^{i i i}$

- For volatile products: $:^{i i}$ The reaction mixture was directly loaded on silica gel (5 g, preconditioned with pentane) column. The crude mixture was first eluted with some pentane to remove benzene, and then with $\mathrm{Et}_{2} \mathrm{O} /$ pentane to isolate the product. ${ }^{i i i}$

\section{Notes:}

- i) Reaction progress monitored by TLC (EtOAc/hexane), visualization: UV (254 nm) and/or PMA stain.

- ii) Use of $\mathrm{DCM}$ or $\mathrm{CHCl}_{3}$ as a solvent during sample preparation for the chiral $\mathrm{GC}$ might lead to product epimerization as these solvents tend to contain $\mathrm{HCl}$ as an impurity.

- iii) Molecular sieves hinder solvent flow when the crude reaction mixture is loaded onto the column. For reactions on a larger scale, a small pad of Celite on top of the silica column was used to prevent column clogging. 


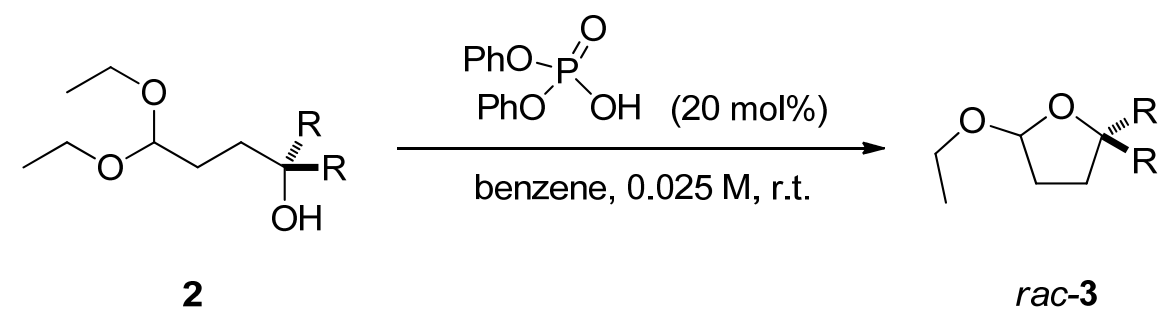

Diphenyl phosphate $(1.25 \mathrm{mg}, 0.005 \mathrm{mmol})$ was added to the solution of $2(0.025 \mathrm{mmol})$ in benzene (1 $\mathrm{ml}$ ). After $1 \mathrm{~h}$ sample of the product for HPLC was isolated by TLC (eluent as used for purification of enantioenriched products).

\section{(R)-5-Ethoxy-2,2-diphenyltetrahydrofuran (3a)}

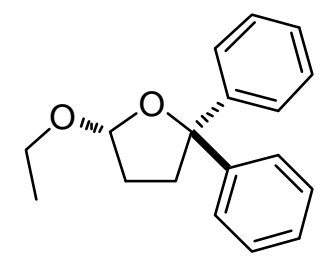

Reaction time: 24 h. Purification: 3\% EtOAc/hexane. Colorless oil, $76.7 \mathrm{mg}, 95 \%$.

${ }^{1} \mathrm{H}$ NMR (400 MHz, $\left.\mathrm{CD}_{2} \mathrm{Cl}_{2}\right) \delta$ 7.46-7.41 (m, 4H), 7.31-7.27 (m, 4H), 7.22-7.17 $(\mathrm{m}, 2 \mathrm{H}), 5.35(\mathrm{dd}, J=5.0,1.9 \mathrm{~Hz}, 1 \mathrm{H}), 3.87-3.80(\mathrm{~m}, 1 \mathrm{H}), 3.57-3.49(\mathrm{~m}, 1 \mathrm{H})$, 2.72-2.65 (m, 1H), 2.61-2.55 (m, 1H) 2.04-1.90 (m, 2H), $1.13(\mathrm{t}, J=7.1 \mathrm{~Hz}, 3 \mathrm{H}) .{ }^{13} \mathrm{C} \mathrm{NMR}(100 \mathrm{MHz}$, $\left.\mathrm{CD}_{2} \mathrm{Cl}_{2}\right) \delta 147.8,147.2,128.35,128.25,126.94,126.92,126.4,126.0,104.5,89.3,63.3,37.5,32.9$, 15.3. HRMS (ESI+) $m / z$ calculated for $\mathrm{C}_{18} \mathrm{H}_{20} \mathrm{O}_{2} \mathrm{Na}\left(\mathrm{M}+\mathrm{Na}^{+}\right)$291.1356, found 291.1358. HPLC (OJ-H), $n$-heptane $/ i$-PrOH 90:10, $0.5 \mathrm{ml} / \mathrm{min}, \lambda=210 \mathrm{~nm}, \mathrm{t}_{\text {minor }}=15.3 \mathrm{~min}, \mathrm{t}_{\text {major }}=28.2 \mathrm{~min}$, er $=94.5: 5.5$.

\section{(R)-5-Ethoxy-2,2-bis(4-methoxyphenyl)tetrahydrofuran (3b)}

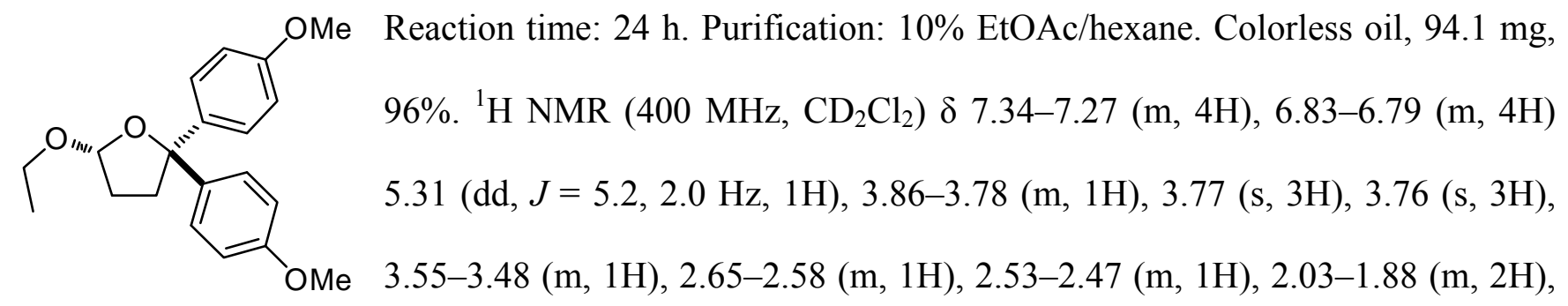

$1.15(\mathrm{t}, J=7.1 \mathrm{~Hz}, 3 \mathrm{H}) .{ }^{13} \mathrm{C} \mathrm{NMR}\left(100 \mathrm{MHz}, \mathrm{CD}_{2} \mathrm{Cl}_{2}\right) \delta 158.70,158.68,140.2,127.7,127.2,113.55$, 113.45, 104.5, 88.9, 63.2, 55.5, 37.8, 33.0, 15.4. HRMS (ESI+) $m / z$ calculated for $\mathrm{C}_{20} \mathrm{H}_{24} \mathrm{O}_{4} \mathrm{Na}\left(\mathrm{M}+\mathrm{Na}^{+}\right)$ 
351.1567, found 351.1563. HPLC (OJ-H), $n$-heptane/i-PrOH 60:40, $0.5 \mathrm{ml} / \mathrm{min}, \lambda=210 \mathrm{~nm}, \mathrm{t}_{\text {minor }}=$ $22.5 \mathrm{~min}, \mathrm{t}_{\text {major }}=32.9 \mathrm{~min}$, er $=95.5: 4.5$.

\section{(R)-5-Ethoxy-2,2-bis(4-fluorophenyl)tetrahydrofuran (3c)}

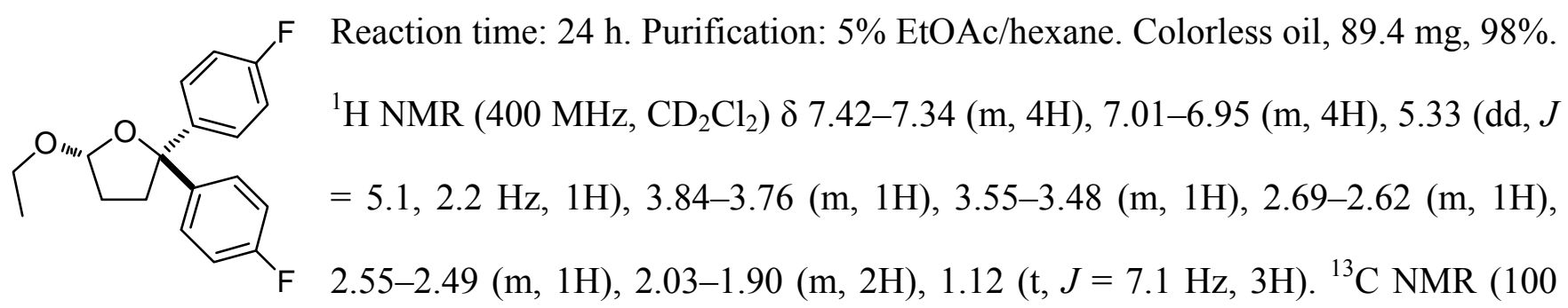

$\left.\mathrm{MHz}, \mathrm{CD}_{2} \mathrm{Cl}_{2}\right) \delta 162.0(\mathrm{~d}, J=244.1 \mathrm{~Hz}), 143.5(\mathrm{~d}, J=3.2 \mathrm{~Hz}), 142.8(\mathrm{~d}, J=3.1 \mathrm{~Hz}), 128.2(\mathrm{~d}, J=8.0$ Hz), $127.8(\mathrm{~d}, J=8.0 \mathrm{~Hz}), 115.0(\mathrm{~d}, J=21.3 \mathrm{~Hz}), 114.9(\mathrm{~d}, J=21.2 \mathrm{~Hz}), 104.6,88.5,63.4,37.9,32.9$, 15.3. HRMS (ESI+) $m / z$ calculated for $\mathrm{C}_{18} \mathrm{H}_{18} \mathrm{O}_{2} \mathrm{~F}_{2} \mathrm{Na}\left(\mathrm{M}+\mathrm{Na}^{+}\right)$327.1167, found 327.1168. HPLC (OJH), $n$-heptane $/ i$-PrOH 98:2, $0.5 \mathrm{ml} / \mathrm{min}, \lambda=210 \mathrm{~nm}, \mathrm{t}_{\text {minor }}=12.3 \mathrm{~min}, \mathrm{t}_{\text {major }}=14.8 \mathrm{~min}$, er $=97: 3$.

\section{(R)-2,2-Bis(4-bromophenyl)-5-ethoxytetrahydrofuran (3d)}

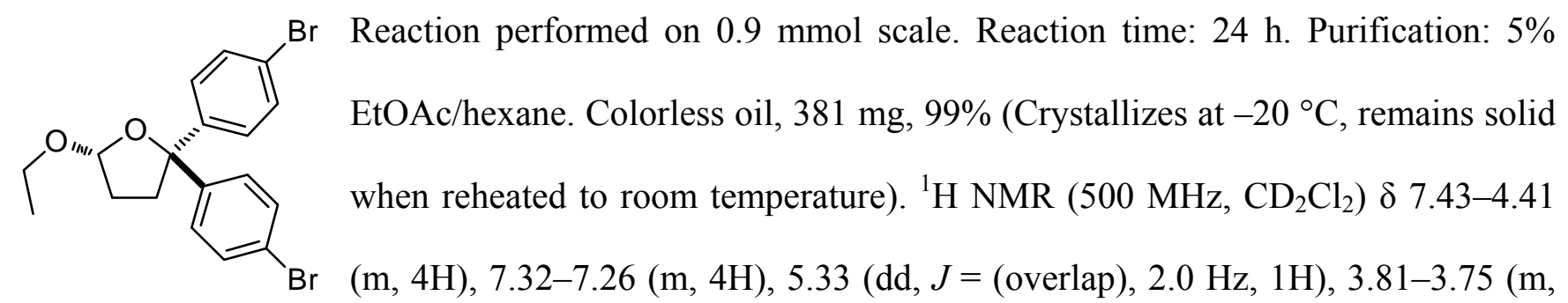

1H), 3.54-3.48 (m, 1H), 2.68-2.62 (m, 1H), 2.53-2.48 (m, 1H), 2.01-1.90 (m, 2H), $1.12(\mathrm{t}, J=7.0 \mathrm{~Hz}$ 3H). ${ }^{13} \mathrm{C}$ NMR $\left(125 \mathrm{MHz}, \mathrm{CD}_{2} \mathrm{Cl}_{2}\right) \delta 146.4,145.8,131.5,131.4,128.3,127.9,121.0,104.6,88.4,63.4$, 37.5, 32.8, 15.3. HRMS (ESI+) $m / z$ calculated for $\mathrm{C}_{18} \mathrm{H}_{18} \mathrm{O}_{2} \mathrm{Na}\left(\mathrm{M}+\mathrm{Na}^{+}\right)$446.9566, found 446.9570. HPLC (OJ-H), $n$-heptane $/ i-P r O H ~ 98: 2,0.5 \mathrm{ml} / \mathrm{min}, \lambda=220 \mathrm{~nm}, \mathrm{t}_{\text {minor }}=11.8 \mathrm{~min}, \mathrm{t}_{\text {major }}=13.0 \mathrm{~min}$, er $=$ $97: 3$. 


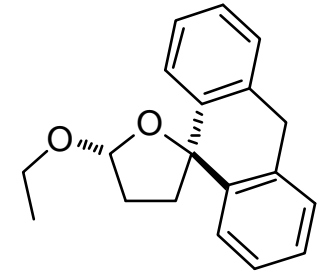

Reaction time: 7 days. Purification: $30 \% \mathrm{CH}_{2} \mathrm{Cl}_{2} /$ hexane. Colorless oil, $72.1 \mathrm{mg}$, $86 \%$. To remove the solvent from the product, the sample was dissolved several

times in $\mathrm{Et}_{2} \mathrm{O}$ and concentrated. Further extensive drying of this product resulted in the formation of a small amount of impurity (see Copies of NMR Spectra). ${ }^{1} \mathrm{H}$ NMR (500 MHz, $\left.\mathrm{CD}_{2} \mathrm{Cl}_{2}\right) \delta 7.76(\mathrm{~d}, J=7.8 \mathrm{~Hz}, 1 \mathrm{H}), 7.45(\mathrm{dd}, J=7.8,1.2 \mathrm{~Hz}, 1 \mathrm{H}), 7.34-7.21(\mathrm{~m}, 6 \mathrm{H}), 5.68(\mathrm{~d}, J=5.0$ Hz, 1H), 4.22-4.15 (m, 1H), 4.01 (d, $J=17.9 \mathrm{~Hz}, 1 \mathrm{H}), 3.92(\mathrm{~d}, J=17.9 \mathrm{~Hz}, 1 \mathrm{H}), 3.79-3.73(\mathrm{~m}, 1 \mathrm{H})$, 2.17-2.10 (m, 1H), 2.10-2.02 (m, 1H), 2.02-1.96 (m, 2H), $1.37(\mathrm{t}, J=7.1 \mathrm{~Hz}, 3 \mathrm{H}) .{ }^{13} \mathrm{C}$ NMR $(125$ $\left.\mathrm{MHz}, \mathrm{CD}_{2} \mathrm{Cl}_{2}\right) \delta 144.3,143.4,134.7,134.6,127.7,127.2,126.9,126.8,126.3,124.2,124.0,105.8,86.1$, 64.0, 37.9, 36.0, 32.0, 15.5. HRMS (ESI+) $m / z$ calculated for $\mathrm{C}_{19} \mathrm{H}_{20} \mathrm{O}_{2} \mathrm{Na}\left(\mathrm{M}+\mathrm{Na}^{+}\right) 303.1355$, found 303.1355. HPLC (OD-3), $n$-heptane $/ i-\operatorname{PrOH} 90: 10,0.5 \mathrm{ml} / \mathrm{min}, \lambda=210 \mathrm{~nm}, \mathrm{t}_{\text {minor }}=5.50 \mathrm{~min}, \mathrm{t}_{\text {major }}=$ $6.58 \mathrm{~min}, \mathrm{er}=95.5: 4.5$.

\section{(R)-5-Ethoxy-2,2-dimethyltetrahydrofuran (3f)}

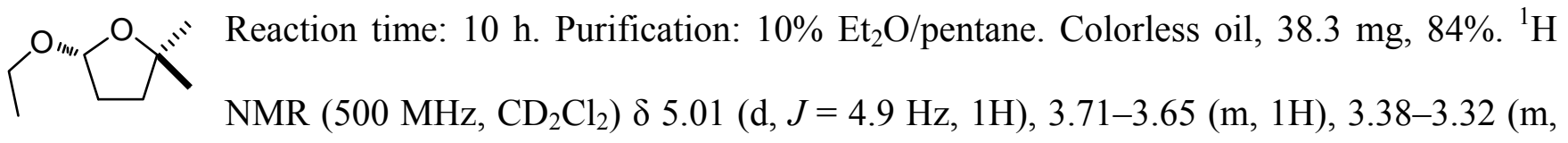
1H), 2.05-1.98 (m, 1H), 1.91-1.84 (m, 2H), 1.71-1.66 (m, 1H), $1.31(\mathrm{~s}, 3 \mathrm{H}), 1.17(\mathrm{~s}, 3 \mathrm{H}), 1.14$ (t, $J=$ $7.0 \mathrm{~Hz}, 3 \mathrm{H}) .{ }^{13} \mathrm{C} \mathrm{NMR}\left(125 \mathrm{MHz}, \mathrm{CD}_{2} \mathrm{Cl}_{2}\right) \delta 104.2,82.1,62.3,36.6,33.4,30.1,28.9,15.4$. HRMS (EI (FE) $i$-butane) $m / z$ calculated for $\mathrm{C}_{8} \mathrm{H}_{17} \mathrm{O}_{2}(\mathrm{M}+\mathrm{H})$ 145.1228, found 145.1227. Chiral GC (Column: 29.5 m BGB-176 SE/SE52 (2,3-dimethyl-6-tert-butyldimethylsilyl- $\beta$-cyclodextrin), i.D. 0.25 mm, df. 0.15 $\mu \mathrm{m}$; Detector: FID; Temperature: injector $230{ }^{\circ} \mathrm{C}$, detector $350{ }^{\circ} \mathrm{C}$, oven $60{ }^{\circ} \mathrm{C}$; gas: 0.6 bar $\left.\mathrm{H}_{2}\right)$, $\mathrm{t}_{\text {minor }}=$ $4.76 \mathrm{~min}, \mathrm{t}_{\text {major }}=5.57 \mathrm{~min}, \mathrm{er}=94.5: 5.5$. 
Reaction time: $16 \mathrm{~h}$. Purification: 3\% EtOAc/hexane. Colorless oil, $47.6 \mathrm{mg}, 92 \% .{ }^{1} \mathrm{H}$ (m, 1H), 1.98-1.90 (m, 1H), 1.86-1.75 (m, 2H), 1.69-1.51 (m, 3H), 1.51-1.38 (m, 2), $1.13(\mathrm{t}, J=7.1$ $\mathrm{Hz}, 3 \mathrm{H}), 0.87(\mathrm{t}, J=7.5 \mathrm{~Hz}, 3 \mathrm{H}), 0.82(\mathrm{t}, J=7.5 \mathrm{~Hz}, 3 \mathrm{H}) .{ }^{13} \mathrm{C} \mathrm{NMR}\left(125 \mathrm{MHz}, \mathrm{CD}_{2} \mathrm{Cl}_{2}\right) \delta 104.0,87.6$, 62.3, 33.3, 33.0, 32.5, 30.8, 15.4, 8.9, 8.7. HRMS (EI (FE) $i$-butane) $m / z$ calculated for $\mathrm{C}_{10} \mathrm{H}_{21} \mathrm{O}_{2}(\mathrm{M}+\mathrm{H})$ 173.1542, found 173.1540. Chiral GC (Column: $29.5 \mathrm{~m}$ BGB-176 SE/SE52 (2,3-dimethyl-6-tertbutyldimethylsilyl- $\beta$-cyclodextrin), i.D. $0.25 \mathrm{~mm}$, df. $0.15 \mu \mathrm{m}$; Detector: FID; Temperature: injector $220{ }^{\circ} \mathrm{C}$, detector $320{ }^{\circ} \mathrm{C}$, oven $90^{\circ} \mathrm{C}$; gas: 0.8 bar $\left.\mathrm{H}_{2}\right), \mathrm{t}_{\text {minor }}=5.49 \mathrm{~min}, \mathrm{t}_{\text {major }}=5.98 \mathrm{~min}$, er $=97: 3$.

\section{(R)-5-Ethoxy-2,2-diisopropyltetrahydrofuran (3h)}

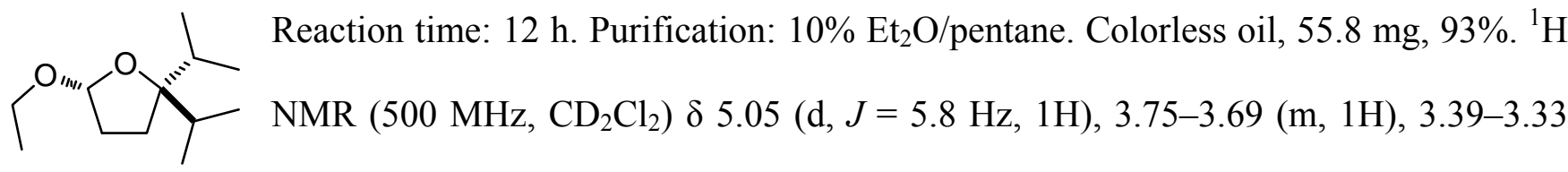
(m, 1H), 2.06-1.99 (m, 1H), 2.00-1.85 (m, 2H), 1.85-1.73 (m, 2H), 1.62-1.57 (m, 1H) $1.14(\mathrm{t}, J=7.1$ $\mathrm{Hz}, 3 \mathrm{H}), 0.91(\mathrm{t}, J=7.6 \mathrm{~Hz}, 6 \mathrm{H}), 0.83(\mathrm{t}, J=6.7 \mathrm{~Hz}, 6 \mathrm{H}) .{ }^{13} \mathrm{C} \mathrm{NMR}\left(125 \mathrm{MHz}, \mathrm{CD}_{2} \mathrm{Cl}_{2}\right) \delta 105.3,93.1$, 62.9, 34.7, 34.3, 33.9, 26.4, 18.4, 18.2, 17.9, 17.7, 15.3. HRMS (ESI+) $m / z$ calculated for $\mathrm{C}_{12} \mathrm{H}_{24} \mathrm{O}_{2} \mathrm{Na}$ $\left(\mathrm{M}+\mathrm{Na}^{+}\right)$223.1669, found 223.1667. Chiral GC (Column: 29.5 m BGB-176 SE/SE52 (2,3-dimethyl-6tert-butyldimethylsilyl- $\beta$-cyclodextrin), i.D. $0.25 \mathrm{~mm}$, df. $0.15 \mu \mathrm{m}$; Detector: FID; Temperature: injector $250{ }^{\circ} \mathrm{C}$, detector $350{ }^{\circ} \mathrm{C}$, oven $100^{\circ} \mathrm{C}$; gas: 0.6 bar $\left.\mathrm{H}_{2}\right), \mathrm{t}_{\text {minor }}=9.02 \mathrm{~min}, \mathrm{t}_{\text {major }}=9.28 \mathrm{~min}$, er $=98: 2$.

\section{(R)-2,2-Di-tert-butyl-5-ethoxytetrahydrofuran (3i)}

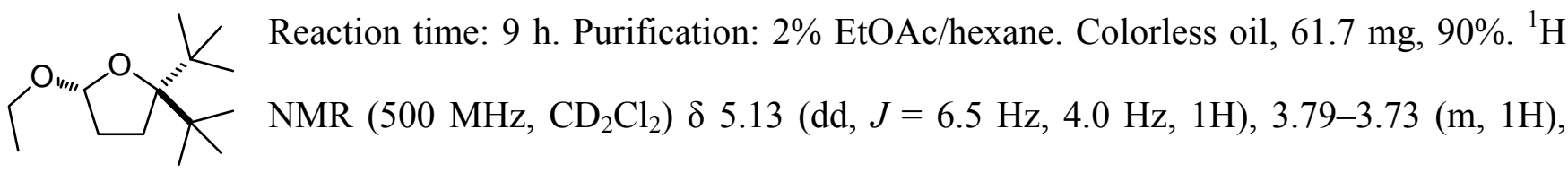
3.45-3.39 (m, 1H), 2.17-2.10 (m, 1H), 1.98-1.92 (m, 1H), 1.86-1.80 (m, 1H), 1.78-1.71 (m, 1H), 1.16 $(\mathrm{t}, J=7.1 \mathrm{~Hz}, 3 \mathrm{H}), 1.06(\mathrm{~s}, 9 \mathrm{H}), 1.00(\mathrm{~s}, 9 \mathrm{H}) .{ }^{13} \mathrm{C} \mathrm{NMR}\left(125 \mathrm{MHz}, \mathrm{CD}_{2} \mathrm{Cl}_{2}\right) \delta 107.0,96.8,63.7,42.5$, 
41.0, 34.7, 29.6, 29.2, 28.7, 15.5. HRMS (ESI+) $m / z$ calculated for $\mathrm{C}_{14} \mathrm{H}_{28} \mathrm{O}_{2} \mathrm{Na}\left(\mathrm{M}+\mathrm{Na}^{+}\right)$251.1981, found 251.1977. Chiral GC (Column: 30 m BGB-176 SE/SE52 (2,3-dimethyl-6-tert-butyldimethylsilyl$\beta$-cyclodextrin), i.D. $0.25 \mathrm{~mm}$, df. $0.25 \mu \mathrm{m}$; Detector: FID; Temperature: injector $230{ }^{\circ} \mathrm{C}$, detector 350 ${ }^{\circ} \mathrm{C}$, oven $90{ }^{\circ} \mathrm{C}$; gas: 0.5 bar $\mathrm{H}_{2}$ ), $\mathrm{t}_{\text {minor }}=65.11 \mathrm{~min}, \mathrm{t}_{\text {major }}=66.38 \mathrm{~min}$, er $=98: 2$.

\section{(R)-2,2-Dibenzyl-5-ethoxytetrahydrofuran (3j)}

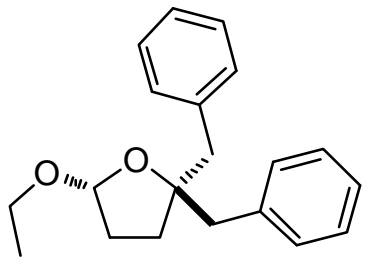

Reaction time: 14 h. Purification: 5\% EtOAc/hexane. Colorless oil, $87.2 \mathrm{mg}$, 98\%. ${ }^{1} \mathrm{H}$ NMR (500 MHz, $\left.\mathrm{CD}_{2} \mathrm{Cl}_{2}\right) \delta$ 7.34-7.29 (m, 4H), 7.25-7.17 (m, 6H), $4.90(\mathrm{~d}, J=5.0 \mathrm{~Hz}, 1 \mathrm{H}), 3.85-3.78(\mathrm{~m}, 1 \mathrm{H}), 3.44-3.37(\mathrm{~m}, 1 \mathrm{H}), 3.04(\mathrm{~d}, J=$ $13.6 \mathrm{~Hz}, 1 \mathrm{H}), 2.94$ (d, $J=13.6 \mathrm{~Hz}, 1 \mathrm{H}), 2.73$ (d, $J=13.6 \mathrm{~Hz}, 1 \mathrm{H}), 2.56(\mathrm{~d}, J=13.6 \mathrm{~Hz}, 1 \mathrm{H}), 1.99-1.93$ $(\mathrm{m}, 1 \mathrm{H}), 1.79-1.75(\mathrm{~m}, 1 \mathrm{H}), 1.56-1.52(\mathrm{~m}, 1 \mathrm{H}), 1.14(\mathrm{t}, J=7.1 \mathrm{~Hz}, 3 \mathrm{H}), 1.02-0.94(\mathrm{~m}, 1 \mathrm{H}) .{ }^{13} \mathrm{C} \mathrm{NMR}$ $\left(125 \mathrm{MHz}, \mathrm{CD}_{2} \mathrm{Cl}_{2}\right) \delta 138.9,138.6,131.0,130.9,128.2,128.1,126.5,126.4,104.8,87.3,62.8,49.2$, 45.3, 33.1, 32.4, 15.4. HRMS (ESI+) $m / z$ calculated for $\mathrm{C}_{20} \mathrm{H}_{24} \mathrm{O}_{2} \mathrm{Na}\left(\mathrm{M}+\mathrm{Na}^{+}\right)$319.1668, found 319.1668. HPLC (OJ-H), $n$-heptane $/ i-\operatorname{PrOH} 98: 2,0.5 \mathrm{ml} / \mathrm{min}, \lambda=220 \mathrm{~nm}, \mathrm{t}_{\text {minor }}=9.6 \mathrm{~min}, \mathrm{t}_{\text {major }}=11.9$ $\min$, er $=97: 3$.

\section{(R)-2-Ethoxy-1-oxaspiro[4.5]decane (3k)}

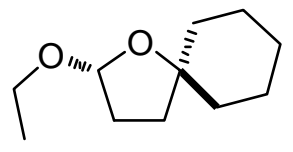

Reaction time: 12 h. Purification: 10\% Et $2 \mathrm{O} /$ pentane. Colorless oil, $52.0 \mathrm{mg}, 94 \% .{ }^{1} \mathrm{H}$ NMR (500 MHz, $\left.\mathrm{CD}_{2} \mathrm{Cl}_{2}\right) \delta 5.01(\mathrm{~d}, J=4.9 \mathrm{~Hz}, 1 \mathrm{H}), 3.72-3.66(\mathrm{~m}, 1 \mathrm{H}), 3.39-3.32$ $(\mathrm{m}, 1 \mathrm{H}), 1.99-1.91(\mathrm{~m}, 1 \mathrm{H}), 1.88-1.83(\mathrm{~m}, 1 \mathrm{H}), 1.81-1.75(\mathrm{~m}, 1 \mathrm{H}), 1.72-1.68(\mathrm{~m}, 1 \mathrm{H}), 1.68-1.61(\mathrm{~m}$, $3 \mathrm{H}), 1.59-1.54(\mathrm{~m}, 1 \mathrm{H}), 1.50-1.46(\mathrm{~m}, 1 \mathrm{H}), 1.43-1.36(\mathrm{~m}, 5 \mathrm{H}), 1.13(\mathrm{t}, J=7.1 \mathrm{~Hz}, 3 \mathrm{H}) .{ }^{13} \mathrm{C} \mathrm{NMR}(125$ $\left.\mathrm{MHz}, \mathrm{CD}_{2} \mathrm{Cl}_{2}\right) \delta 103.6,84.2,62.2,40.1,38.2,34.4,32.8,26.0,24.3,15.5$. HRMS (EI (FE)) $m / z$ calculated for $\mathrm{C}_{11} \mathrm{H}_{20} \mathrm{O}_{2}$ (M) 184.1463, found 184.1465. Chiral GC (Column: 29.5 m BGB-176 SE/SE52 (2,3-dimethyl-6-tert-butyldimethylsilyl- $\beta$-cyclodextrin), i.D. $0.25 \mathrm{~mm}$, df. $0.25 \mu \mathrm{m}$; Detector: FID; 
Temperature: injector $220{ }^{\circ} \mathrm{C}$, detector $320{ }^{\circ} \mathrm{C}$, oven $90{ }^{\circ} \mathrm{C}$; gas: 0.8 bar $\mathrm{H}_{2}$ ), $\mathrm{t}_{\text {minor }}=10.06 \mathrm{~min}, \mathrm{t}_{\text {major }}=$ $10.34 \mathrm{~min}, \mathrm{er}=97: 3$.

(1'S,5R)-5-Ethoxy-2',3',4,5-tetrahydro-3H-spiro[furan-2,1'-indene] + (1'R,5R)-5-ethoxy-2',3',4,5tetrahydro-3H-spiro[furan-2,1'-indene] (3l)

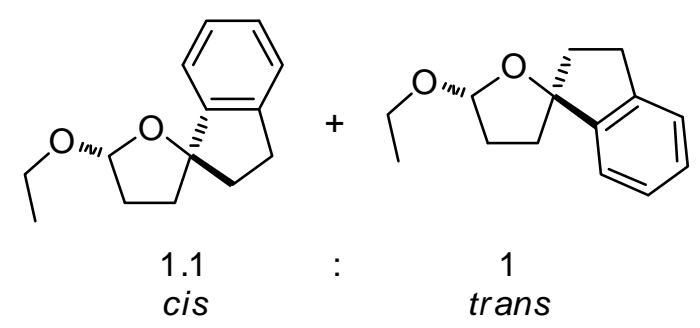

Reaction time: 20 h. Purification: 8\% EtOAc/hexane. Colorless oil, $62.9 \mathrm{mg}, 96 \% .{ }^{1} \mathrm{H}$ NMR (500 MHz, $\left.\mathrm{CD}_{2} \mathrm{Cl}_{2}\right) \delta$ 7.36-7.33 (m, 0.49H, major isomer), 7.25-7.18 (m, 3.26H), $5.26(\mathrm{dd}, J=5.3,2.0 \mathrm{~Hz}, 0.45 \mathrm{H}$, minor isomer), 5.17 (dd, $J=$ 4.1, $0.9 \mathrm{~Hz}, 0.50 \mathrm{H}$, major isomer), 3.80-3.73 (m, 1H), 3.48-3.40 (m, 1H), 3.00-2.91 (m, 1H), 2.85-2.76 (m, 1H), 2.36-2.27 (m, 1H), 2.25-2.01 (m, 5H), $1.23(\mathrm{t}, J=7.1 \mathrm{~Hz}, 1.56 \mathrm{H}$, major isomer), 1.19 (t, $J=$ 7.1 Hz, 1.41H, minor isomer). ${ }^{13} \mathrm{C} \mathrm{NMR}\left(125 \mathrm{MHz}, \mathrm{CD}_{2} \mathrm{Cl}_{2}\right) \delta 147.8,147.1,143.6,143.2,128.3,128.0$, $127.1,126.9,125.0,124.8,124.0,123.0,104.6,103.9,93.8,92.8,63.0,62.7,41.4,40.7,36.3,36.1$, $33.9,33.8,30.2,29.7,15.5,15.4$. Diastereomeric ratio $=1.1: 1$, determined by $1 \mathrm{H}$ NMR, protons on acetal carbon stereocenter, $\delta 5.26(0.4519 \mathrm{H})$ and $\delta 5.17(0.4957 \mathrm{H})$. Relative stereochemistry of diastereomers determined by NOESY experiment: only one of the acetal protons, the one of minor diastereomer $(\delta 5.26)$ has a cross-peak with aromatic proton indicating trans relationship between OEt and aromatic substituent (see the copy of NOESY spectra). HRMS (ESI+) $\mathrm{m} / \mathrm{z}$ calculated for $\mathrm{C}_{14} \mathrm{H}_{18} \mathrm{O}_{2} \mathrm{Na}\left(\mathrm{M}+\mathrm{Na}^{+}\right)$241.1199, found 241.1198. Chiral GC for cis diastereomer (Column: $30 \mathrm{~m}$ Chiraldex G-BP (gamma-cyclodextrin-butyryl), i.D. $0.25 \mathrm{~mm}$, df. $0.25 \mu \mathrm{m}$; Detector: FID; Temperature: injector $220^{\circ} \mathrm{C}$, detector $350{ }^{\circ} \mathrm{C}$, oven $80^{\circ} \mathrm{C}$; gas: 0.9 bar $\mathrm{H}_{2}$ ), $\mathrm{t}_{\text {major }}=211.3 \mathrm{~min}, \mathrm{t}_{\text {minor }}=225.1 \mathrm{~min}$, er $($ cis $)=91: 9$. Chiral GC for trans diastereomer (Column: $24.8 \mathrm{~m}$ Hydrodex-B-TBDAc-CD (Heptakis(2,3-di-O-acetyl-6-O-t-butyldimethyl-sylil)- $\beta$-cyclodextrin), i.D. $0.25 \mathrm{~mm}$; Detector: FID; Temperature: injector $220^{\circ} \mathrm{C}$, detector $350{ }^{\circ} \mathrm{C}$, oven $100{ }^{\circ} \mathrm{C}$; gas: 0.5 bar $\mathrm{H}_{2}$ ), $\mathrm{t}_{\text {major }}=109.0$ min, $\mathrm{t}_{\text {minor }}=112.4$ min, er $($ trans $)=96: 4$. 


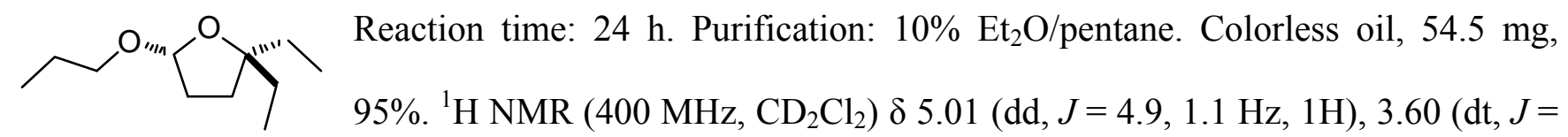
9.4, $6.8 \mathrm{~Hz}, 1 \mathrm{H}), 3.25(\mathrm{dt}, J=9.4,6.6 \mathrm{~Hz}, 1 \mathrm{H}), 1.99-1.90(\mathrm{~m}, 1 \mathrm{H}), 1.88-1.82(\mathrm{~m}, 1 \mathrm{H}), 1.82-1.74(\mathrm{~m}$, $1 \mathrm{H}), 1.70-1.51(\mathrm{~m}, 5 \mathrm{H}), 1.51-1.38(\mathrm{~m}, 2 \mathrm{H}), 0.90(\mathrm{t}, J=7.4 \mathrm{~Hz}, 3 \mathrm{H}), 0.87(\mathrm{t}, J=7.5 \mathrm{~Hz}, 3 \mathrm{H}), 0.82(\mathrm{t}, J$ $=7.5 \mathrm{~Hz}, 3 \mathrm{H}) .{ }^{13} \mathrm{C} \mathrm{NMR}\left(100 \mathrm{MHz}, \mathrm{CD}_{2} \mathrm{Cl}_{2}\right) \delta 104.4,87.7,68.8,33.4,33.0,32.7,30.8,23.4,10.9,9.0$, 8.7. HRMS (CI (FE) $i$-butane) $m / z$ calculated for $\mathrm{C}_{11} \mathrm{H}_{23} \mathrm{O}_{2}(\mathrm{M}+\mathrm{H})$ 187.1698, found 187.1697. Chiral GC (Column: 30 m BGB-177/BGB-15 (2,6-dimethyl-3-pentyl- $\beta$-cyclodextrin), i.D. 0.25 mm, df. 0.25 $\mu \mathrm{m}$; Detector: FID; Temperature: injector $220^{\circ} \mathrm{C}$, detector $350{ }^{\circ} \mathrm{C}$, oven $115^{\circ} \mathrm{C}$; gas: 0.6 bar $\mathrm{H}_{2}$ ), $\mathrm{t}_{\text {minor }}$ $=7.06 \mathrm{~min}, \mathrm{t}_{\text {major }}=7.34 \mathrm{~min}, \mathrm{er}=95: 5$.

\section{(R)-6-Ethoxy-2,2-bis(4-fluorophenyl)tetrahydro-2H-pyran (3n)}

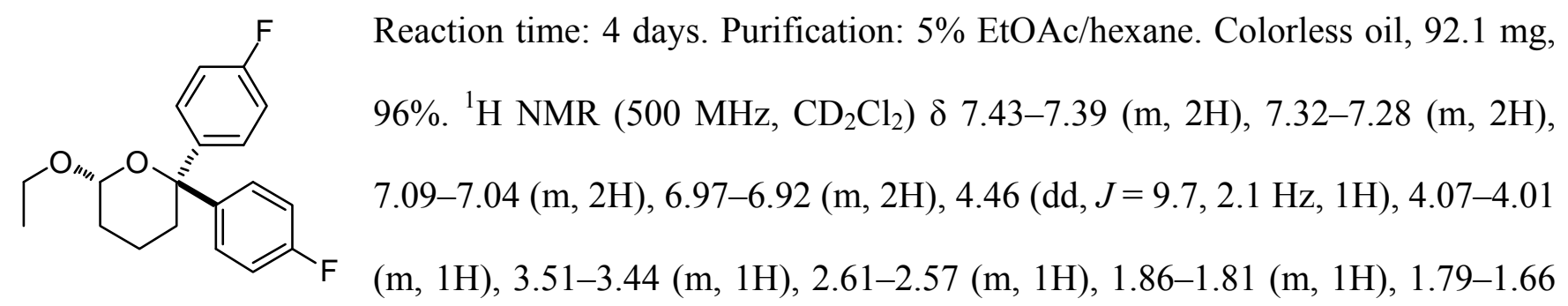

$(\mathrm{m}, 3 \mathrm{H}), 1.54-1.46(\mathrm{~m}, 1 \mathrm{H}), 1.21(\mathrm{t}, J=7.0 \mathrm{~Hz}, 3 \mathrm{H}) .{ }^{13} \mathrm{C} \mathrm{NMR}\left(125 \mathrm{MHz}, \mathrm{CD}_{2} \mathrm{Cl}_{2}\right) \delta 162.1(\mathrm{~d}, J=$ $246.2 \mathrm{~Hz}), 161.7(\mathrm{~d}, J=243.9 \mathrm{~Hz}), 145.2(\mathrm{~d}, J=2.7 \mathrm{~Hz}), 139.4(\mathrm{~d}, J=3.3 \mathrm{~Hz}), 129.2(\mathrm{~d}, J=8.1 \mathrm{~Hz})$, $126.9(\mathrm{~d}, J=7.7 \mathrm{~Hz}), 115.7(\mathrm{~d}, J=21.8 \mathrm{~Hz}), 114.9$ (d, $J=21.1 \mathrm{~Hz}), 97.9,80.3,64.1,34.9,31.5,20.1$, 15.5. HRMS (EI (DE)) $m / z$ calculated for $\mathrm{C}_{19} \mathrm{H}_{20} \mathrm{O}_{2} \mathrm{~F}_{2}(\mathrm{M}) 318.1431$, found 318.1431. HPLC (OJ-H), $n$ heptane $/ i-\mathrm{PrOH} 99.7: 0.3,0.5 \mathrm{ml} / \mathrm{min}, \lambda=220 \mathrm{~nm}, \mathrm{t}_{\text {minor }}=13.9 \mathrm{~min}, \mathrm{t}_{\text {major }}=17.3 \mathrm{~min}, \mathrm{er}=82: 18$. 


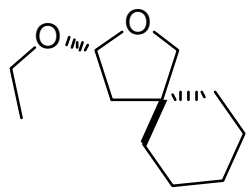

Reaction time: $30 \mathrm{~min}$. Purification: 10\% Et $2 \mathrm{O} /$ pentane. Colorless oil, $51.9 \mathrm{mg}, 94 \%$.

${ }^{1} \mathrm{H}$ NMR $\left(500 \mathrm{MHz}, \mathrm{CD}_{2} \mathrm{Cl}_{2}\right) \delta 5.07(\mathrm{dd}, J=5.8,2.6 \mathrm{~Hz}, 1 \mathrm{H}), 3.70-3.64(\mathrm{~m}, 1 \mathrm{H}), 3.60$ $(\mathrm{d}, J=8.3 \mathrm{~Hz}, 1 \mathrm{H}), 3.56(\mathrm{~d}, J=8.3 \mathrm{~Hz}, 1 \mathrm{H}), 3.41-3.34(\mathrm{~m}, 1 \mathrm{H}), 1.89(\mathrm{dd}, J=13.3,5.7$

$\mathrm{Hz}, 1 \mathrm{H}), 1.60(\mathrm{dd}, J=13.3,2.6 \mathrm{~Hz}, 1 \mathrm{H}), 1.53-1.36(\mathrm{~m}, 10 \mathrm{H}), 1.15(\mathrm{t}, J=7.1 \mathrm{~Hz}, 3 \mathrm{H}) .{ }^{13} \mathrm{C}$ NMR $(125$ $\left.\mathrm{MHz}, \mathrm{CD}_{2} \mathrm{Cl}_{2}\right) \delta 104.7,77.1,63.1,45.5,43.1,37.2,36.4,26.3,24.5,23.9,15.5$. HRMS (ESI+) $\mathrm{m} / \mathrm{z}$ calculated for $\mathrm{C}_{11} \mathrm{H}_{20} \mathrm{O}_{2} \mathrm{Na}\left(\mathrm{M}+\mathrm{Na}^{+}\right)$207.1356, found 207.1355. Chiral GC (Column: 29.5m BGB-176 SE/SE52 (2,3-dimethyl-6-tert-butyldimethylsilyl- $\beta$-cyclodextrin), i.D. $0.25 \mathrm{~mm}$, df. $0.15 \mu \mathrm{m}$; Detector: FID; Temperature: injector $220^{\circ} \mathrm{C}$, detector $350{ }^{\circ} \mathrm{C}$, oven $110^{\circ} \mathrm{C}$; gas: 0.5 bar $\mathrm{H}_{2}$ ), $\mathrm{t}_{\text {minor }}=14.64$ min, $\mathrm{t}_{\text {major }}=15.19 \min , \mathrm{er}=82.5: 17.5$.

\section{(R)-2-Ethoxytetrahydrofuran (3p)}

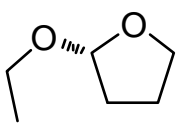

Reaction time: $7 \mathrm{~h}$. Purification: product is highly volatile but it can be isolated as a solution in organic solvent (e.g. dichloromethane): $(1 / 6(2 \mathrm{ml})$ of the reaction mixture was directly loaded on silica gel ( $0.5 \mathrm{~g}$, preconditioned with pentane) column and product eluted with $\mathrm{CD}_{2} \mathrm{Cl}_{2}$.). Yield 76\%: determined by ${ }^{1} \mathrm{H}$ NMR using $\mathrm{Ph}_{3} \mathrm{CH}(0.05 \mathrm{mmol})$ as internal standard and comparing relative area of $\mathrm{Ph}_{3} \mathrm{CH}$ and proton on acetal carbon stereocenter. ${ }^{1} \mathrm{H} \mathrm{NMR}(500 \mathrm{MHz}$, $\left.\mathrm{CD}_{2} \mathrm{Cl}_{2}\right) \delta 5.08(\mathrm{dd}, J=4.7,1.4 \mathrm{~Hz}, 1 \mathrm{H}), 3.85-3.78(\mathrm{~m}, 2 \mathrm{H}), 3.69-3.63(\mathrm{~m}, 1 \mathrm{H}), 3.42-3.36(\mathrm{~m}, 1 \mathrm{H})$, $1.97-1.86(\mathrm{~m}, 2 \mathrm{H}), 1.84-1.76(\mathrm{~m}, 2 \mathrm{H}), 1.14(\mathrm{t}, J=7.0 \mathrm{~Hz}, 3 \mathrm{H}) .{ }^{13} \mathrm{C} \mathrm{NMR}\left(125 \mathrm{MHz}, \mathrm{CD}_{2} \mathrm{Cl}_{2}\right) \delta 103.9$, 67.0, 62.7, 32.7, 23.9, 15.4. GC-MS (EI) m/z 116. Chiral GC (Column: 30 m BGB-176 SE/SE52 (2,3dimethyl-6-tert-butyldimethylsilyl- $\beta$-cyclodextrin), i.D. $0.25 \mathrm{~mm}$, df. $0.25 \mu \mathrm{m}$; Detector: FID; Temperature: injector $220^{\circ} \mathrm{C}$, detector $320^{\circ} \mathrm{C}$, oven $70^{\circ} \mathrm{C}$; gas: 0.5 bar $\left.\mathrm{H}_{2}\right), \mathrm{t}_{\text {minor }}=5.20$ min, $\mathrm{t}_{\text {major }}=$ $5.79 \mathrm{~min}, \mathrm{er}=79: 21$. 


\section{X-Ray Data for Compound 3d}

Absolute configuration determination of 3d by X-ray single-crystal structure analysis

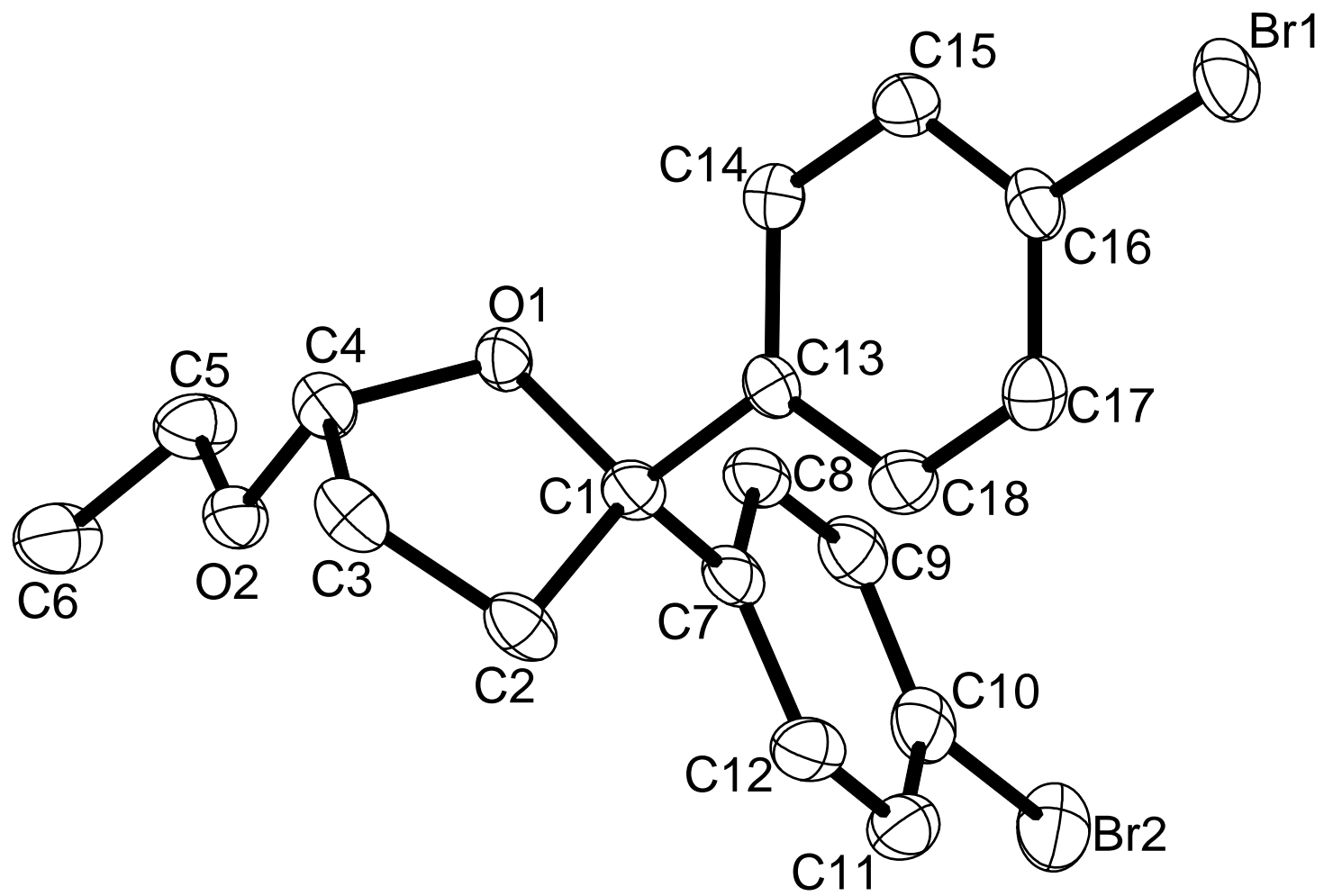

Figure 1. Crystal structure of 3d, showing the absolute configuration of the sample. Anisotropic displacement parameters are drawn at the $50 \%$ probablility level and hydrogen atoms omitted for clarity.

Crystals of $\mathbf{3 d}$ were obtained as fine needles after keeping the oil in the freezer at $-20{ }^{\circ} \mathrm{C}$. The compound remains solid when warmed up to room temperature. More crystals in the form of prisms were obtained by dissolving a few milligrams of the product in dichloromethane, evaporating the solvent, and keeping the remaining oil at $-20{ }^{\circ} \mathrm{C}$ overnight. The absolute configurations of four crystals picked at random from the sample were investigated, and all showed the configuration shown in Figure 1. The Flack parameter $\mathrm{x}$ and the Hooft factor $\mathrm{y}$ for the four crystals were as follows: (1) $\mathrm{x}=-0.018(12)$ (3048 reflections), $\mathrm{y}=-0.026$ (4) (1447 Bijvoet pairs) $(\mathrm{Cu}) ;(2) \mathrm{x}=-0.009(11)$ (6792 reflections), $\mathrm{y}=-$ 0.0120(1) (3304 Bijvoet pairs)(Mo); (3) $\mathrm{x}=-0.07(4)$ (2168 reflections), $\mathrm{y}=-0.096(22)$ (1036 Bijvoet pairs $)(\mathrm{Cu}) ;(4) \mathrm{x}=-0.003(20)(2797$ reflections $), \mathrm{y}=-0.004(12)(1226$ Bijvoet pairs $)(\mathrm{Cu})$. 
X-ray Crystal Structure Analysis of 3d: $\left[\mathrm{C}_{18} \mathrm{H}_{18} \mathrm{Br}_{2} \mathrm{O}_{2}\right], M_{r}=426.14 \mathrm{~g} \cdot \mathrm{mol}^{-1}$, colorless prism, crystal size $0.060 \times 0.204 \times 0.573 \mathrm{~mm}^{3}$, tetragonal, space group P4 $1, a=10.8373(9) \AA, c=14.8921(13) \AA, V=$ 1749.0(3) $\AA^{3}, T=100 \mathrm{~K}, Z=4, D_{\text {calc }}=1.618 \mathrm{~g} \cdot \mathrm{cm}^{3}, \lambda=1.54178 \AA, \mu=5.909 \mathrm{~mm}^{-1}$, Gaussian absorption correction $\left(\mathrm{T}_{\min }=0.10636, \mathrm{~T}_{\max }=0.70728\right)$, scaling SADABS, Bruker AXS ProteumX8 diffractometer, $4.08<\theta<66.52^{\circ}, 40020$ measured reflections, 3048 independent reflections, 3046 reflections with $I>2 \sigma(I), R_{\text {int }}=0.0405$. Structure solved by direct methods and refined by full-matrix least-squares against $F^{2}$ to $R_{1}=0.0168[I>2 \sigma(I)], w R_{2}=0.0407,200$ parameters. $^{2}$ The Flack parameter is $-0.018(12) .{ }^{3}$ The Hooft factor y based on 1447 Bijvoet pairs is $-0.026(4){ }^{4} \mathrm{H}$ atoms riding, $S=1.133$, residual electron density $+0.26 /-0.22$ e $\AA^{-3}$. CCDC 771853 .

The supplementary crystallographic data for 3d (CCDC 771853 can be obtained free of charge from The Cambridge Crystallographic Data Centre via www.ccdc.cam.ac.uk/data_request/cif.

${ }^{2}$ Sheldrick, G. M. Acta Cryst. 2008, A64, 112.

${ }^{3}$ Flack, H. D. Acta Cryst. 1983, A39, 876.

${ }^{4}$ Hooft, R. W. W.; Straver, L. H.; Spek, A. L. J. Appl. Cryst. 2008, 41, 96. 
Table 1. Crystal data and structure refinement.

Empirical formula

Color

Formula weight

Temperature

Wavelength

Crystal system

Space group

Unit cell dimensions

Volume

$\mathrm{Z}$

Density (calculated)

Absorption coefficient

$\mathrm{F}(000)$

Crystal size

$\theta$ range for data collection

Index ranges

Reflections collected

Independent reflections

Reflections with $\mathrm{I}>2 \sigma(\mathrm{I})$

Completeness to $\theta=66.52^{\circ}$

Absorption correction

Max. and min. transmission

Refinement method

Data / restraints / parameters

Goodness-of-fit on $\mathrm{F}^{2}$

Final R indices $[\mathrm{I}>2 \sigma(\mathrm{I})]$

$\mathrm{R}$ indices (all data)

Absolute structure parameter

Largest diff. peak and hole
$\mathrm{C}_{18} \mathrm{H}_{18} \mathrm{Br}_{2} \mathrm{O}_{2}$

colourless

$426.14 \mathrm{~g} \cdot \mathrm{mol}^{-1}$

$100 \mathrm{~K}$

$1.54178 \AA$

Tetragonal

P4, (no. 76)

$\mathrm{a}=10.8373(9) \AA \quad \alpha=90^{\circ}$.

$\mathrm{b}=10.8373(9) \AA \quad \beta=90^{\circ}$.

$\mathrm{c}=14.8921(13) \AA \quad \gamma=90^{\circ}$.

$1749.0(3) \AA^{3}$

4

$1.618 \mathrm{Mg} \cdot \mathrm{m}^{-3}$

$5.909 \mathrm{~mm}^{-1}$

848 e

$0.973 \times 0.204 \times 0.060 \mathrm{~mm}^{3}$

4.08 to $66.52^{\circ}$.

$-12 \leq \mathrm{h} \leq 12,-12 \leq \mathrm{k} \leq 12,-17 \leq 1 \leq 17$

40020

$3048\left[\mathrm{R}_{\text {int }}=0.0405\right]$

3046

$99.6 \%$

Gaussian

0.71 and 0.11

Full-matrix least-squares on $\mathrm{F}^{2}$

3048 / 1 / 200

1.133

$\mathrm{R}_{1}=0.0168 \quad \mathrm{wR}^{2}=0.0407$

$\mathrm{R}_{1}=0.0168 \quad \mathrm{wR}^{2}=0.0407$

$-0.018(12)$

0.256 and $-0.216 \mathrm{e} \cdot \AA^{-3}$ 
Table 2. Atomic coordinates and equivalent isotropic displacement parameters $\left(\AA^{2}\right)$. $\mathrm{U}_{\mathrm{eq}}$ is defined as one third of the trace of the orthogonalized $\mathrm{U}_{\mathrm{ij}}$ tensor.

\begin{tabular}{|c|c|c|c|c|}
\hline & $\mathrm{X}$ & $\mathrm{y}$ & $\mathrm{Z}$ & $\mathrm{U}_{\mathrm{eq}}$ \\
\hline $\operatorname{Br}(1)$ & $0.0872(1)$ & $-0.3557(1)$ & $1.1153(1)$ & $0.033(1)$ \\
\hline $\operatorname{Br}(2)$ & $0.3290(1)$ & $0.1452(1)$ & $0.5970(1)$ & $0.036(1)$ \\
\hline $\mathrm{C}(1)$ & $0.2644(2)$ & $0.1691(2)$ & $1.0105(2)$ & $0.022(1)$ \\
\hline $\mathrm{C}(2)$ & $0.1805(2)$ & $0.2743(2)$ & $1.0443(2)$ & $0.026(1)$ \\
\hline$C(3)$ & $0.2454(2)$ & $0.3136(2)$ & $1.1306(2)$ & $0.028(1)$ \\
\hline$C(4)$ & $0.3788(2)$ & $0.2997(2)$ & $1.1047(2)$ & $0.024(1)$ \\
\hline$C(5)$ & $0.5412(2)$ & $0.4053(2)$ & $1.0296(2)$ & $0.033(1)$ \\
\hline$C(6)$ & $0.5751(2)$ & $0.5339(2)$ & $1.0007(2)$ & $0.038(1)$ \\
\hline$C(7)$ & $0.2797(2)$ & $0.1664(2)$ & $0.9089(2)$ & $0.023(1)$ \\
\hline $\mathrm{C}(8)$ & $0.3915(2)$ & $0.1288(2)$ & $0.8713(2)$ & $0.024(1)$ \\
\hline $\mathrm{C}(9)$ & $0.4060(2)$ & $0.1220(2)$ & $0.7788(2)$ & $0.027(1)$ \\
\hline$C(10)$ & $0.3082(2)$ & $0.1537(2)$ & $0.7241(2)$ & $0.028(1)$ \\
\hline $\mathrm{C}(11)$ & $0.1959(2)$ & $0.1907(2)$ & $0.7589(2)$ & $0.031(1)$ \\
\hline$C(12)$ & $0.1829(2)$ & $0.1961(2)$ & $0.8519(2)$ & $0.027(1)$ \\
\hline$C(13)$ & $0.2198(2)$ & $0.0419(2)$ & $1.0420(1)$ & $0.020(1)$ \\
\hline $\mathrm{C}(14)$ & $0.2959(2)$ & $-0.0374(2)$ & $1.0902(2)$ & $0.024(1)$ \\
\hline$C(15)$ & $0.2555(2)$ & $-0.1547(2)$ & $1.1144(2)$ & $0.026(1)$ \\
\hline$C(16)$ & $0.1389(2)$ & $-0.1914(2)$ & $1.0901(1)$ & $0.025(1)$ \\
\hline$C(17)$ & $0.0600(2)$ & $-0.1141(2)$ & $1.0436(2)$ & $0.026(1)$ \\
\hline $\mathrm{C}(18)$ & $0.1011(2)$ & $0.0015(2)$ & $1.0195(2)$ & $0.025(1)$ \\
\hline $\mathrm{O}(1)$ & $0.3837(1)$ & $0.1913(1)$ & $1.0508(1)$ & $0.023(1)$ \\
\hline $\mathrm{O}(2)$ & $0.4137(1)$ & $0.4050(1)$ & $1.0552(1)$ & $0.025(1)$ \\
\hline
\end{tabular}


Table 3. Bond lengths $[\AA \AA]$ and angles $\left[^{\circ}\right]$.

\begin{tabular}{|c|c|c|c|c|}
\hline $\operatorname{Br}(1)-C(16)$ & $1.9047(19)$ & $\operatorname{Br}(2)-C(10)$ & $1.908(2)$ & $C(1)-$ \\
\hline $\mathrm{O}(1)$ & $1.446(2)$ & $\mathrm{C}(1)-\mathrm{C}(7)$ & $1.523(3)$ & $C(1)-$ \\
\hline$C(13)$ & $1.534(3)$ & $\mathrm{C}(1)-\mathrm{C}(2)$ & $1.543(3)$ & $C(2)-$ \\
\hline $\mathrm{C}(3)$ & $1.526(3)$ & $\mathrm{C}(3)-\mathrm{C}(4)$ & $1.504(3)$ & $C(4)-$ \\
\hline $\mathrm{O}(2)$ & $1.411(3)$ & $\mathrm{C}(4)-\mathrm{O}(1)$ & $1.424(3)$ & $C(5)-$ \\
\hline $\mathrm{O}(2)$ & $1.434(3)$ & $\mathrm{C}(5)-\mathrm{C}(6)$ & $1.504(3)$ & $C(7)-$ \\
\hline$C(12)$ & $1.387(3)$ & $\mathrm{C}(7)-\mathrm{C}(8)$ & $1.395(3)$ & $C(8)-$ \\
\hline $\mathrm{C}(9)$ & $1.389(4)$ & $\mathrm{C}(9)-\mathrm{C}(10)$ & $1.379(3)$ & $C(10)-$ \\
\hline $\mathrm{C}(11)$ & $1.382(3)$ & $\mathrm{C}(11)-\mathrm{C}(12)$ & $1.394(4)$ & $C(13)-$ \\
\hline$C(14)$ & $1.390(3)$ & $\mathrm{C}(13)-\mathrm{C}(18)$ & $1.399(3)$ & $C(14)-$ \\
\hline$C(15)$ & $1.392(3)$ & $\mathrm{C}(15)-\mathrm{C}(16)$ & $1.374(3)$ & $C(16)-$ \\
\hline$C(17)$ & $1.383(3)$ & $\mathrm{C}(17)-\mathrm{C}(18)$ & $1.377(3)$ & \\
\hline $\mathrm{O}(1)-\mathrm{C}(1)-\mathrm{C}(7)$ & $108.57(16)$ & $\mathrm{O}(1)-\mathrm{C}(1)-\mathrm{C}(13)$ & $107.74(16)$ & $C(7)-$ \\
\hline$C(1)-C(13)$ & $108.74(17)$ & $\mathrm{O}(1)-\mathrm{C}(1)-\mathrm{C}(2)$ & $105.59(17)$ & $C(7)-$ \\
\hline$C(1)-C(2)$ & $113.69(17)$ & $C(13)-C(1)-C(2)$ & $112.25(17)$ & $C(3)-$ \\
\hline $\mathrm{C}(2)-\mathrm{C}(1)$ & $102.06(17)$ & $\mathrm{C}(4)-\mathrm{C}(3)-\mathrm{C}(2)$ & $101.53(18)$ & $\mathrm{O}(2)-$ \\
\hline $\mathrm{C}(4)-\mathrm{O}(1)$ & $111.24(18)$ & $\mathrm{O}(2)-\mathrm{C}(4)-\mathrm{C}(3)$ & $108.08(16)$ & $\mathrm{O}(1)-$ \\
\hline$C(4)-C(3)$ & $105.23(16)$ & $\mathrm{O}(2)-\mathrm{C}(5)-\mathrm{C}(6)$ & $108.22(18)$ & $C(12)-$ \\
\hline$C(7)-C(8)$ & $118.6(2)$ & $\mathrm{C}(12)-\mathrm{C}(7)-\mathrm{C}(1)$ & 121.41(19) & $C(8)-$ \\
\hline$C(7)-C(1)$ & $119.90(19)$ & $\mathrm{C}(9)-\mathrm{C}(8)-\mathrm{C}(7)$ & $120.8(2)$ & $C(10)-$ \\
\hline $\mathrm{C}(9)-\mathrm{C}(8)$ & $119.0(2)$ & $\mathrm{C}(9)-\mathrm{C}(10)-\mathrm{C}(11)$ & $121.8(2)$ & $C(9)-$ \\
\hline$C(10)-\operatorname{Br}(2)$ & $118.83(18)$ & $C(11)-C(10)-B r(2)$ & $119.32(18)$ & $C(10)-$ \\
\hline$C(11)-C(12)$ & $118.3(2)$ & $C(7)-C(12)-C(11)$ & $121.4(2)$ & $C(14)-$ \\
\hline $\mathrm{C}(13)-\mathrm{C}(18)$ & $118.39(18)$ & $\mathrm{C}(14)-\mathrm{C}(13)-\mathrm{C}(1)$ & $121.73(18)$ & $C(18)-$ \\
\hline $\mathrm{C}(13)-\mathrm{C}(1)$ & 119.84(18) & $\mathrm{C}(13)-\mathrm{C}(14)-\mathrm{C}(15)$ & $120.78(19)$ & $C(16)-$ \\
\hline$C(15)-C(14)$ & $119.03(19)$ & $C(15)-C(16)-C(17)$ & $121.70(18)$ & $C(15)-$ \\
\hline $\mathrm{C}(16)-\mathrm{Br}(1)$ & $119.33(16)$ & $\mathrm{C}(17)-\mathrm{C}(16)-\mathrm{Br}(1)$ & $118.89(15)$ & $C(18)-$ \\
\hline$C(17)-C(16)$ & 118.79(19) & $\mathrm{C}(17)-\mathrm{C}(18)-\mathrm{C}(13)$ & $121.3(2)$ & $C(4)-$ \\
\hline $\mathrm{O}(1)-\mathrm{C}(1)$ & $109.78(14)$ & $\mathrm{C}(4)-\mathrm{O}(2)-\mathrm{C}(5)$ & $113.50(15)$ & \\
\hline
\end{tabular}


Table 4. Anisotropic displacement parameters $\left(\AA^{2}\right)$.

The anisotropic displacement factor exponent takes the form:

$-2 \pi^{2}\left[h^{2} a^{* 2} U_{11}+\ldots+2 h k a^{*} b^{*} U_{12}\right]$.

\begin{tabular}{|c|c|c|c|c|c|}
\hline $\mathrm{U}_{11}$ & $\mathrm{U}_{22}$ & $\mathrm{U}_{33}$ & $\mathrm{U}_{23}$ & $\mathrm{U}_{13}$ & $\mathrm{U}_{12}$ \\
\hline $\mathrm{Br}(1) 0.046(1)$ & $0.021(1)$ & $0.032(1)$ & $0.003(1)$ & $0.000(1)$ & $-0.009(1)$ \\
\hline $\operatorname{Br}(2) 0.051(1)$ & $0.032(1)$ & $0.026(1)$ & $0.005(1)$ & $0.000(1)$ & $-0.013(1)$ \\
\hline $\mathrm{C}(1) \quad 0.015(1)$ & $0.020(1)$ & $0.032(1)$ & $0.001(1)$ & $0.000(1)$ & $0.001(1)$ \\
\hline $\mathrm{C}(2) \quad 0.021(1)$ & $0.020(1)$ & $0.039(1)$ & $0.002(1)$ & $0.007(1)$ & $0.003(1)$ \\
\hline $\mathrm{C}(3) \quad 0.033(1)$ & $0.021(1)$ & $0.030(1)$ & $0.002(1)$ & $0.009(1)$ & $0.002(1)$ \\
\hline $\mathrm{C}(4) \quad 0.029(1)$ & $0.020(1)$ & $0.023(1)$ & $0.000(1)$ & $0.002(1)$ & $0.001(1)$ \\
\hline $\mathrm{C}(5) \quad 0.022(1)$ & $0.031(1)$ & $0.045(2)$ & $0.011(1)$ & $-0.005(1)$ & $-0.003(1)$ \\
\hline$C(6) \quad 0.029(1)$ & $0.034(1)$ & $0.051(2)$ & $0.016(1)$ & $-0.007(1)$ & $-0.007(1)$ \\
\hline $\mathrm{C}(7) \quad 0.022(1)$ & $0.015(1)$ & $0.031(1)$ & $0.004(1)$ & $0.000(1)$ & $-0.002(1)$ \\
\hline $\mathrm{C}(8) \quad 0.019(1)$ & $0.021(1)$ & $0.032(1)$ & $0.002(1)$ & $-0.001(1)$ & $0.001(1)$ \\
\hline $\mathrm{C}(9) \quad 0.027(1)$ & $0.021(1)$ & $0.033(1)$ & $0.002(1)$ & $0.003(1)$ & $-0.002(1)$ \\
\hline$C(10) 0.036(1)$ & $0.021(1)$ & $0.028(1)$ & $0.004(1)$ & $0.000(1)$ & $-0.009(1)$ \\
\hline $\mathrm{C}(11) 0.030(1)$ & $0.027(1)$ & $0.034(1)$ & $0.008(1)$ & $-0.008(1)$ & $-0.006(1)$ \\
\hline $\mathrm{C}(12) 0.020(1)$ & $0.025(1)$ & $0.037(1)$ & $0.006(1)$ & $-0.001(1)$ & $-0.001(1)$ \\
\hline$C(13) 0.022(1)$ & $0.018(1)$ & $0.021(1)$ & $-0.003(1)$ & $0.003(1)$ & $0.001(1)$ \\
\hline$C(14) 0.024(1)$ & $0.022(1)$ & $0.027(1)$ & $-0.001(1)$ & $-0.003(1)$ & $-0.002(1)$ \\
\hline$C(15) 0.033(1)$ & $0.024(1)$ & $0.022(1)$ & $0.001(1)$ & $-0.004(1)$ & $0.002(1)$ \\
\hline$C(16) 0.034(1)$ & $0.017(1)$ & $0.022(1)$ & $0.000(1)$ & $0.002(1)$ & $-0.003(1)$ \\
\hline$C(17) 0.022(1)$ & $0.026(1)$ & $0.029(1)$ & $-0.002(1)$ & $0.002(1)$ & $-0.004(1)$ \\
\hline $\mathrm{C}(18) 0.020(1)$ & $0.025(1)$ & $0.029(1)$ & $0.000(1)$ & $0.000(1)$ & $0.001(1)$ \\
\hline $\mathrm{O}(1) \quad 0.018(1)$ & $0.018(1)$ & $0.032(1)$ & $-0.004(1)$ & $-0.002(1)$ & $0.000(1)$ \\
\hline $\mathrm{O}(2) \quad 0.023(1)$ & $0.020(1)$ & $0.033(1)$ & $0.004(1)$ & $-0.003(1)$ & $-0.002(1)$ \\
\hline
\end{tabular}




\section{Copies of NMR Spectra}
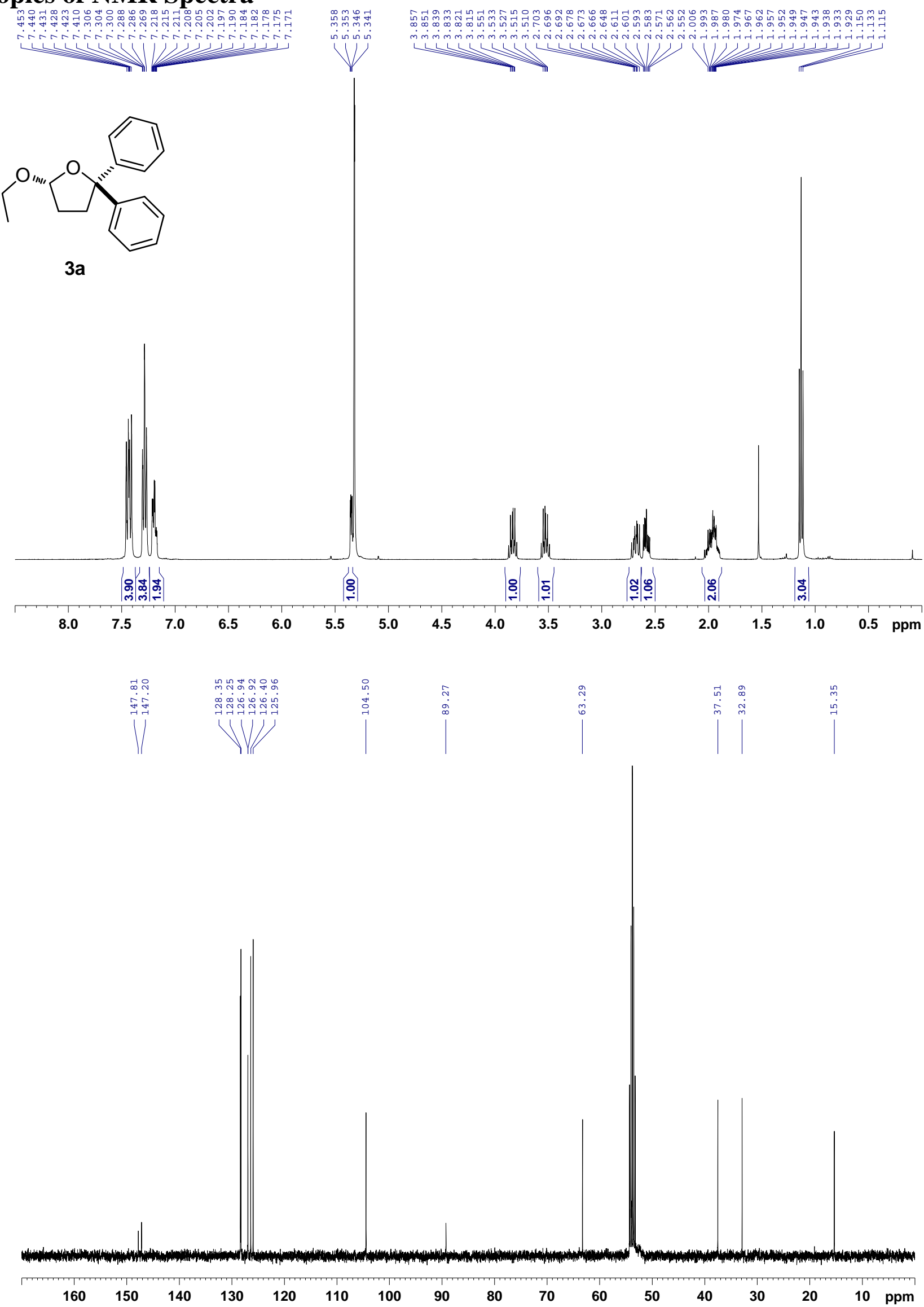


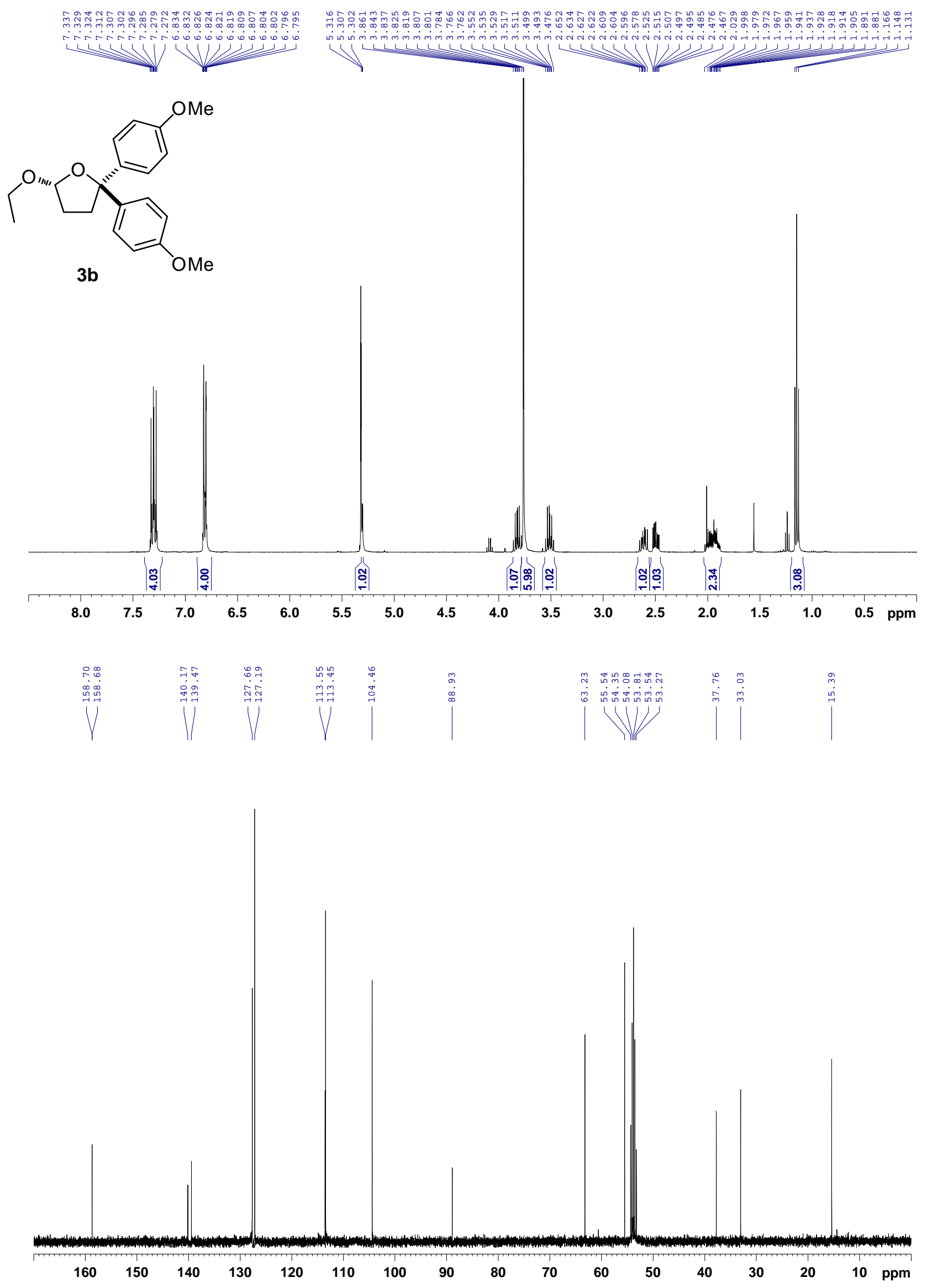




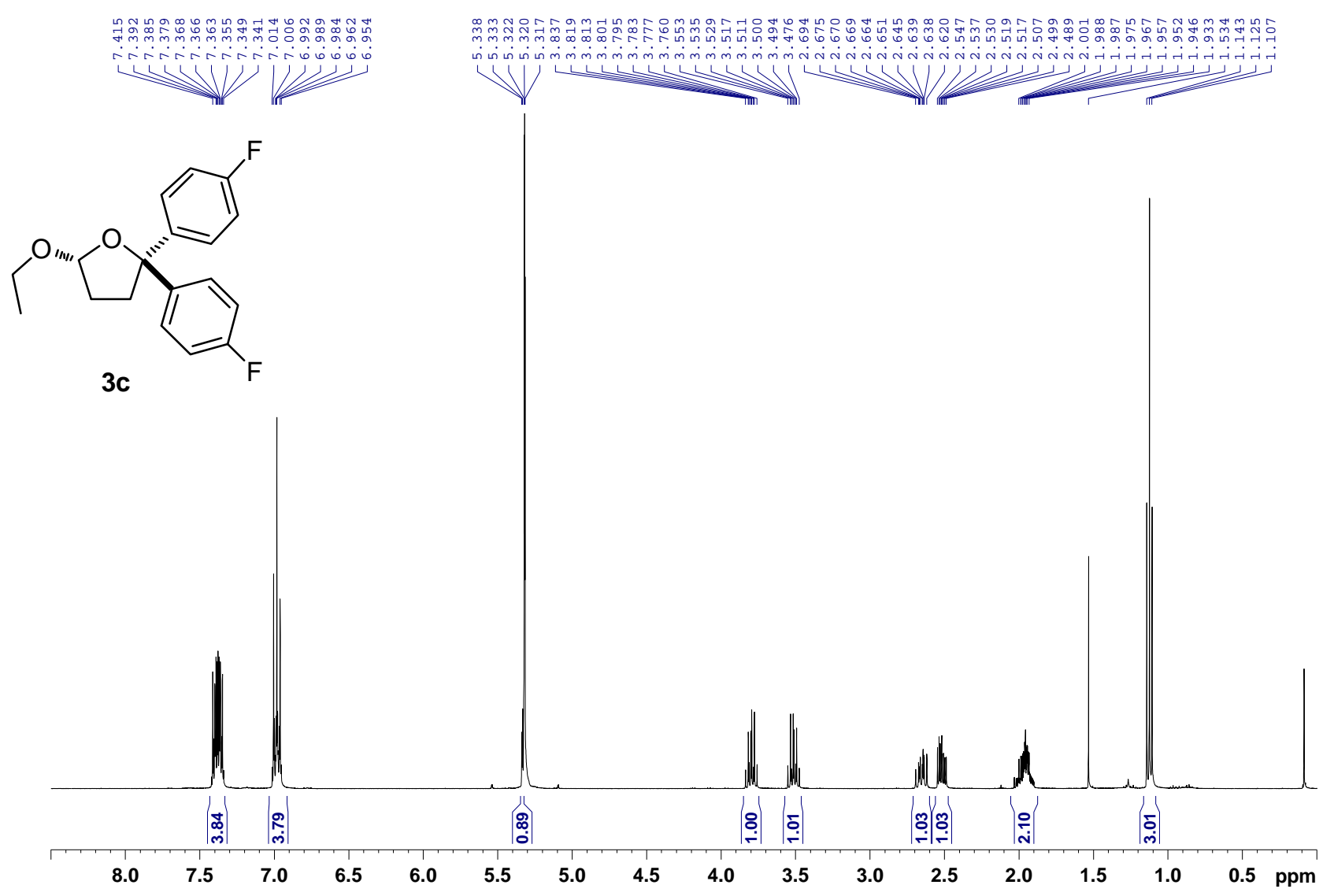

|

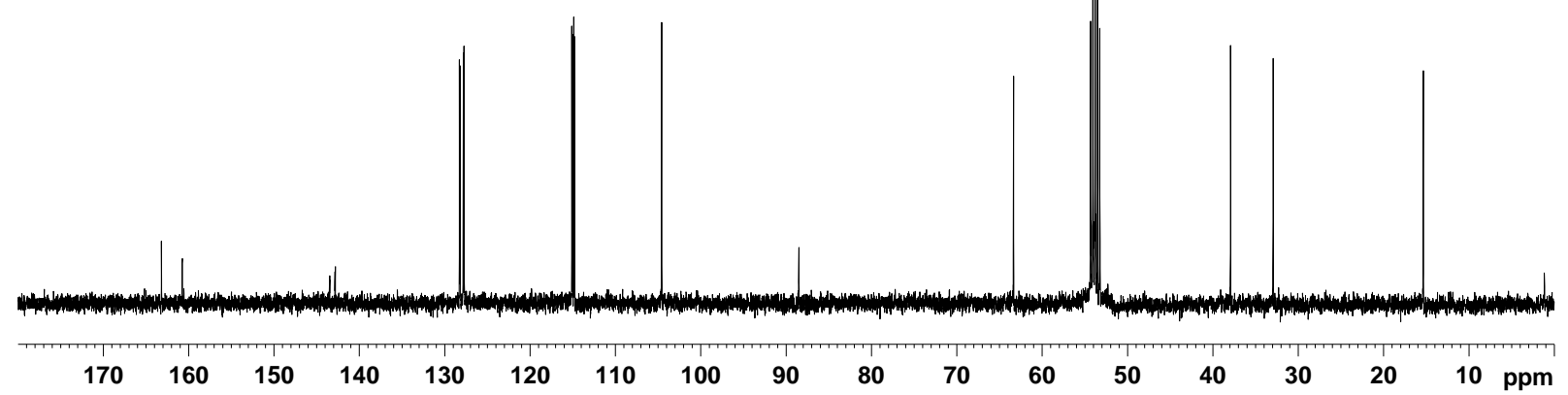


<smiles>CCO[C@@H]1CCC(c2ccc(Br)cc2)(c2ccc(Br)cc2)O1</smiles>

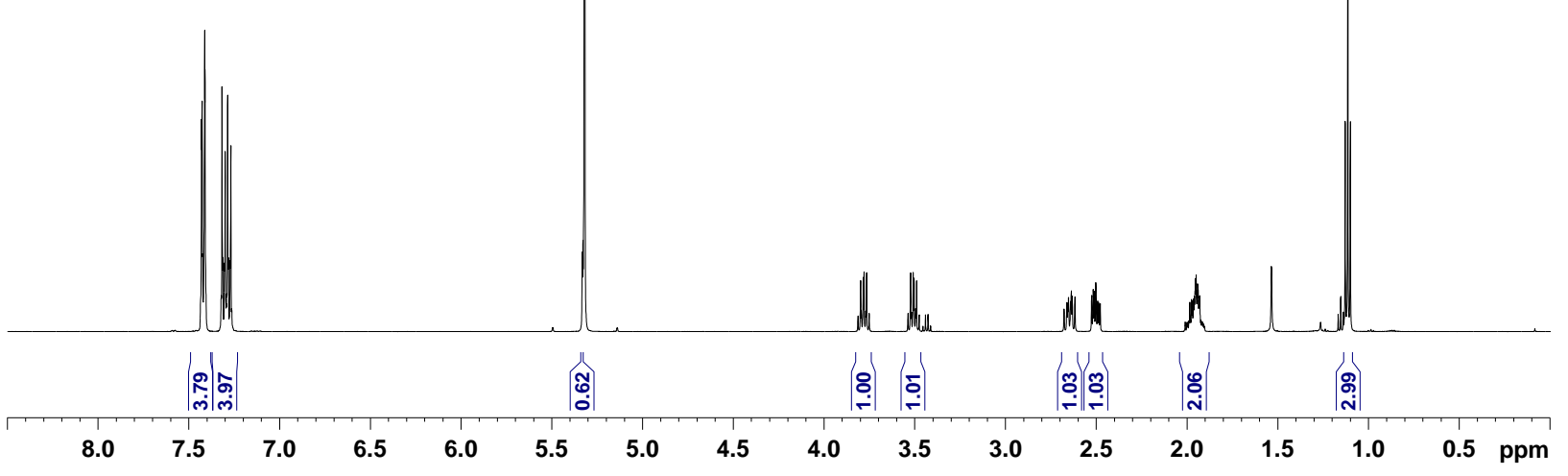

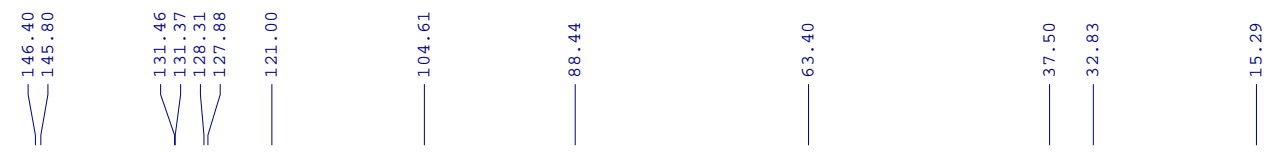

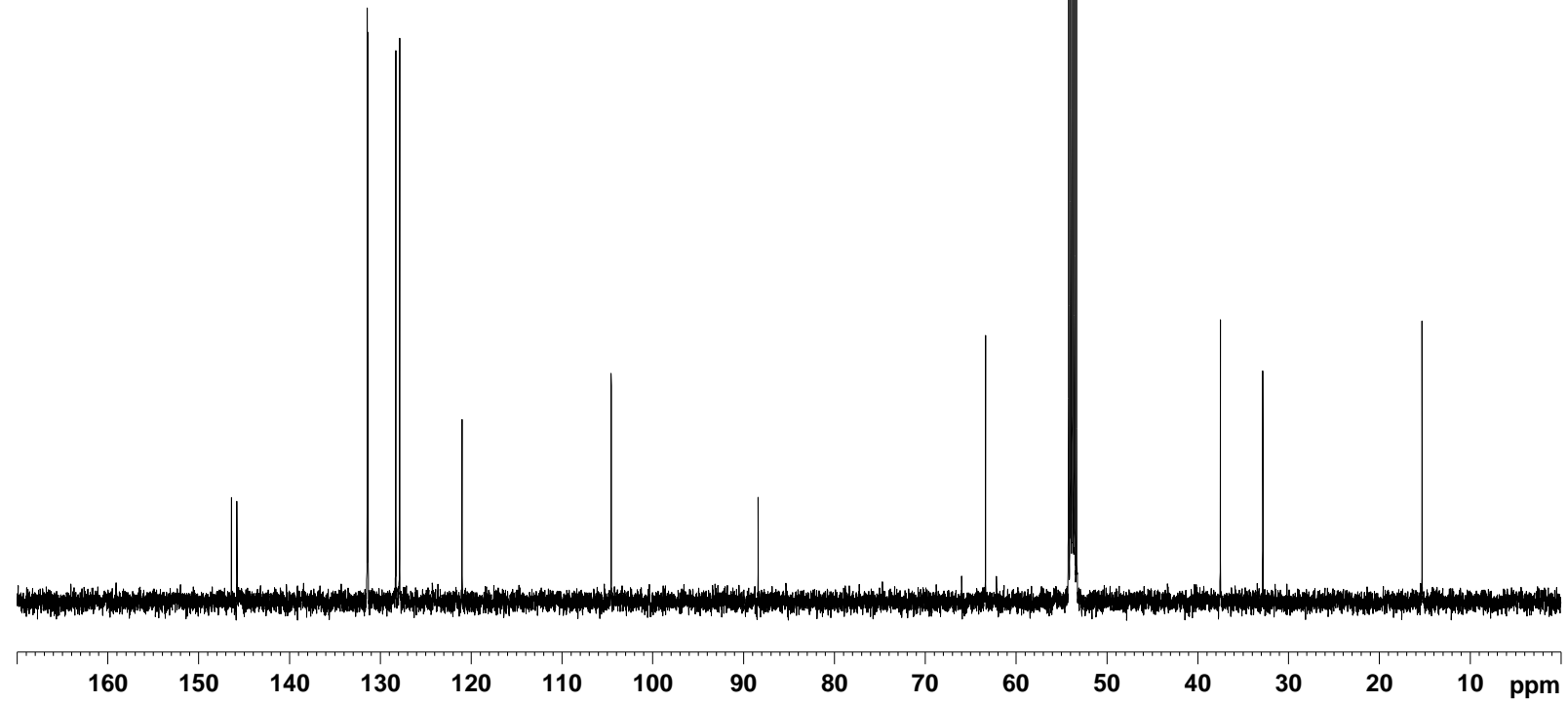


<smiles>CCO[C@H]1CC[C@]2(Cc3ccccc32)c2ccccc21</smiles>

(some $\mathrm{Et}_{2} \mathrm{O}$ present)

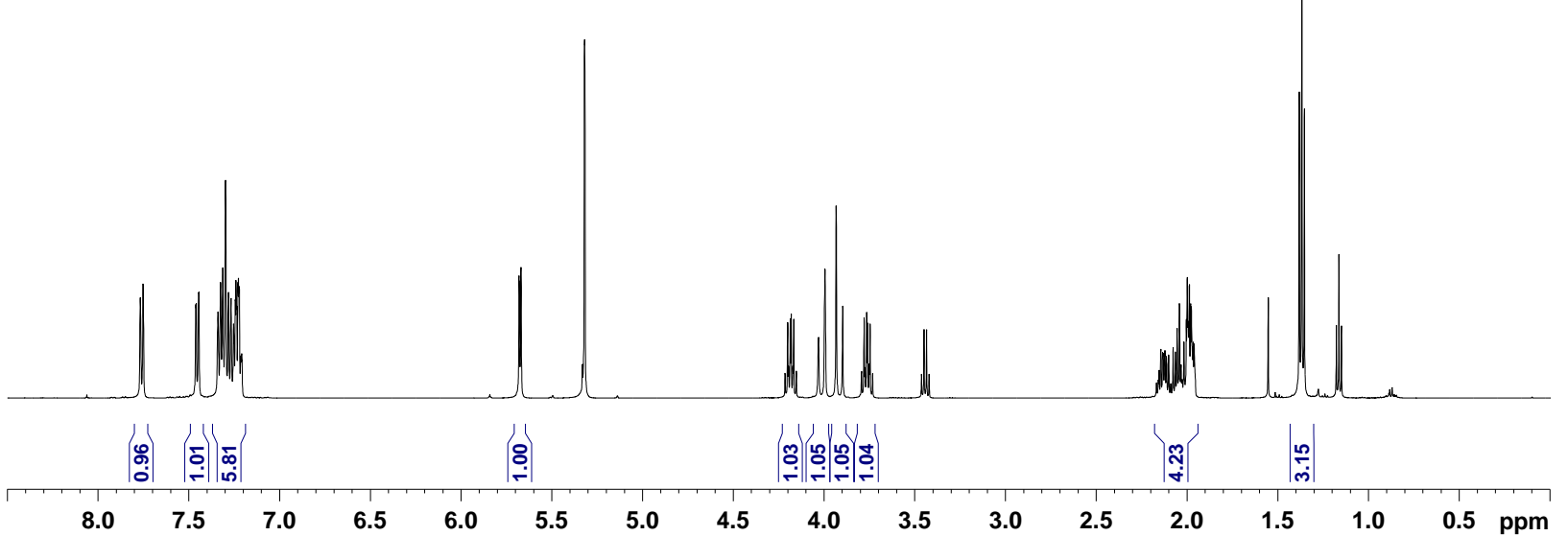

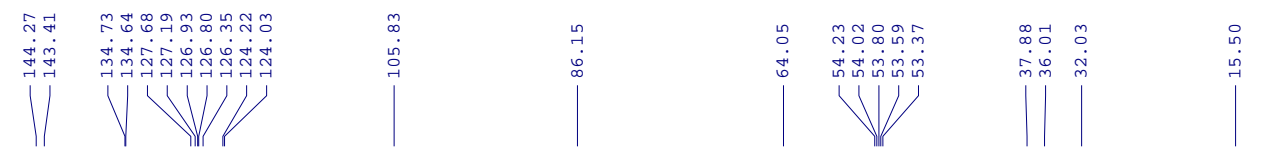

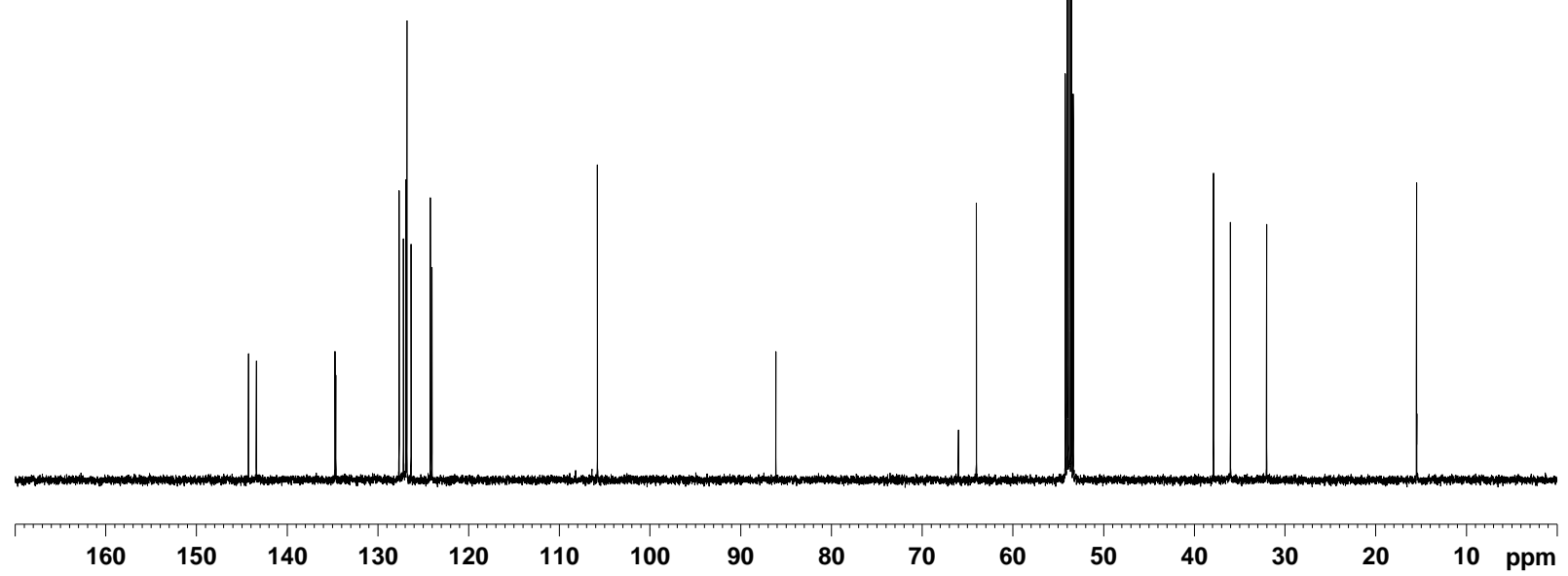




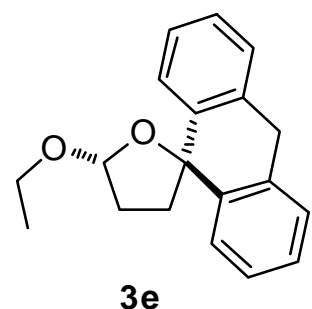

(extensive drying resulted in the formation of a small amount of impurity)
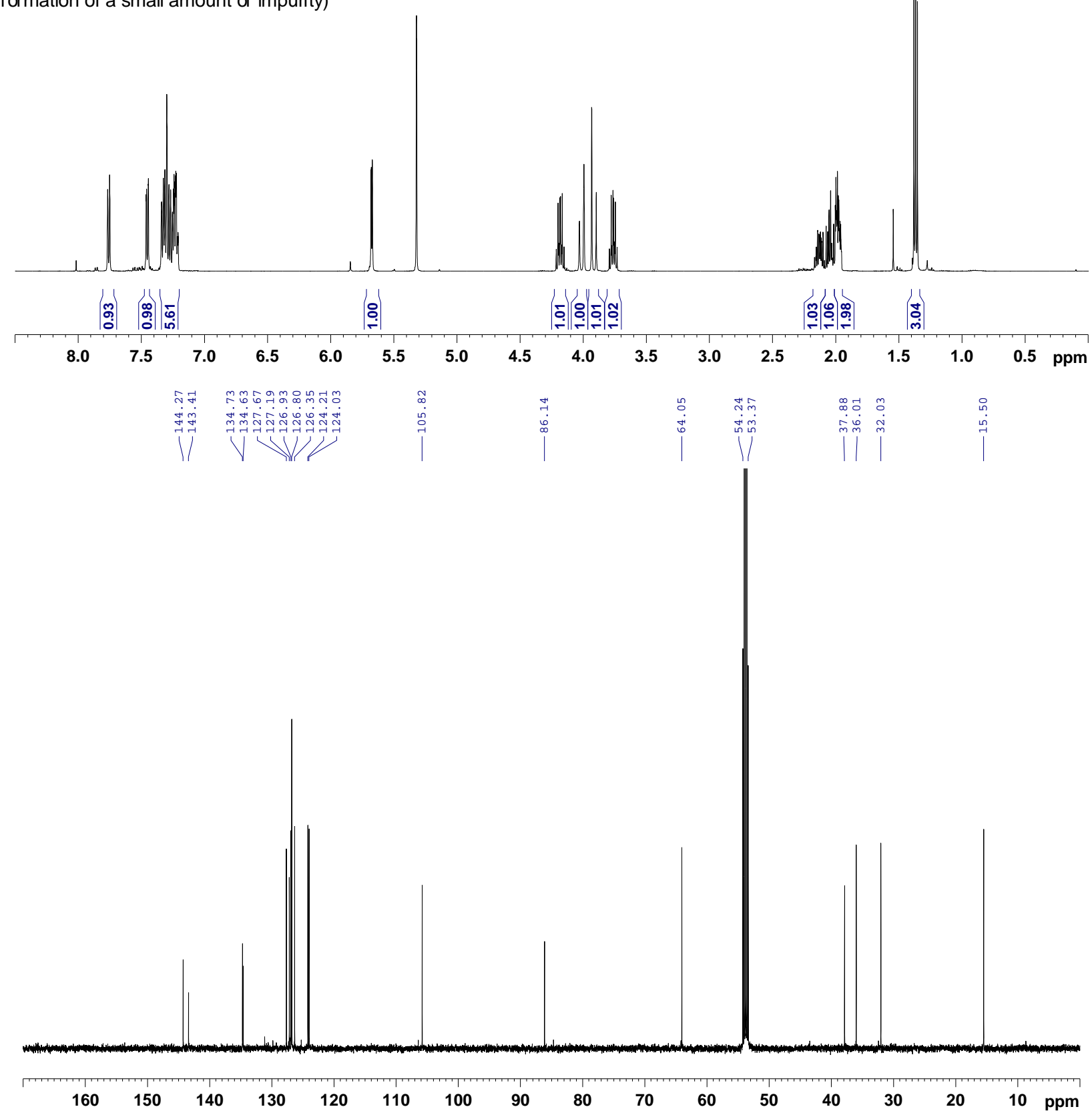

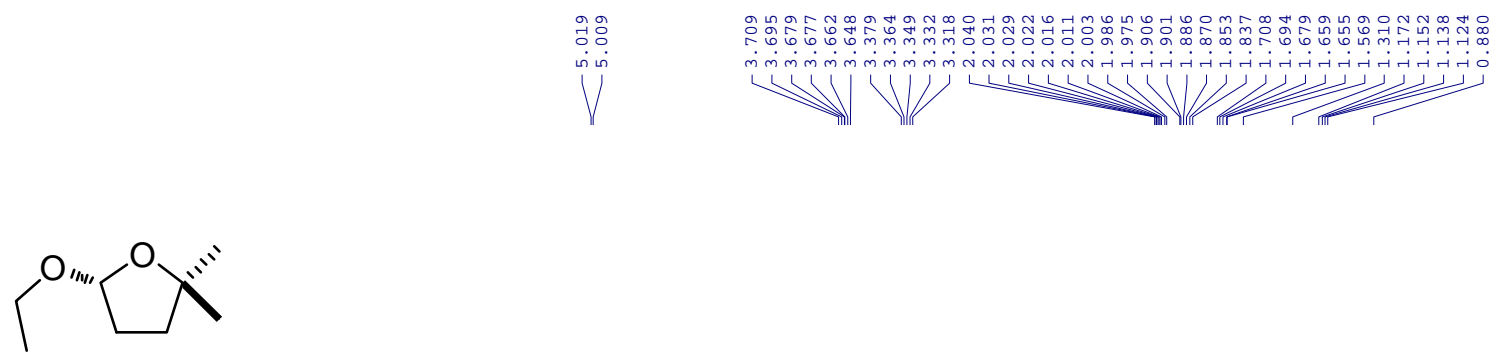

$3 f$

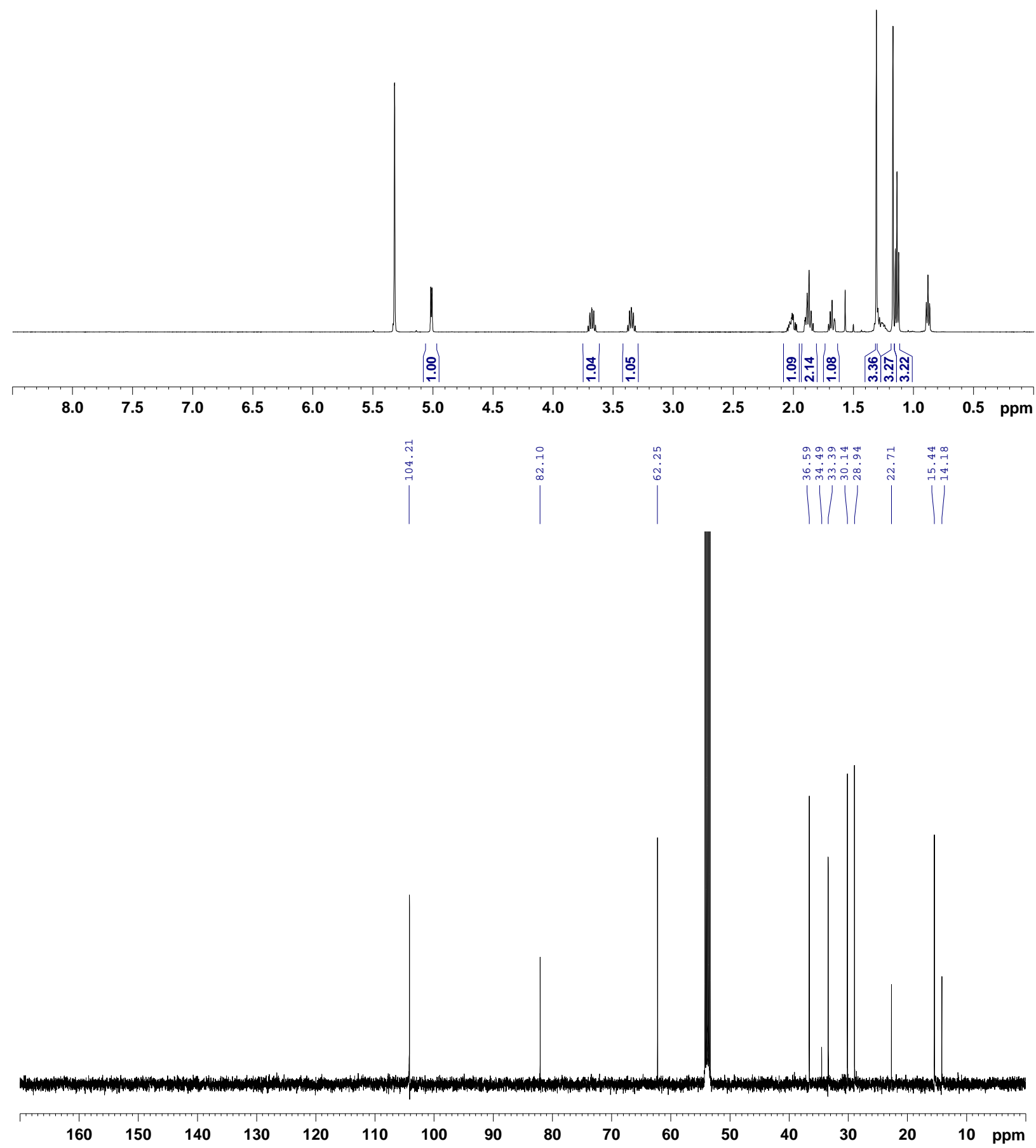




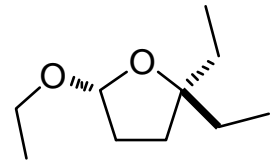

$3 g$
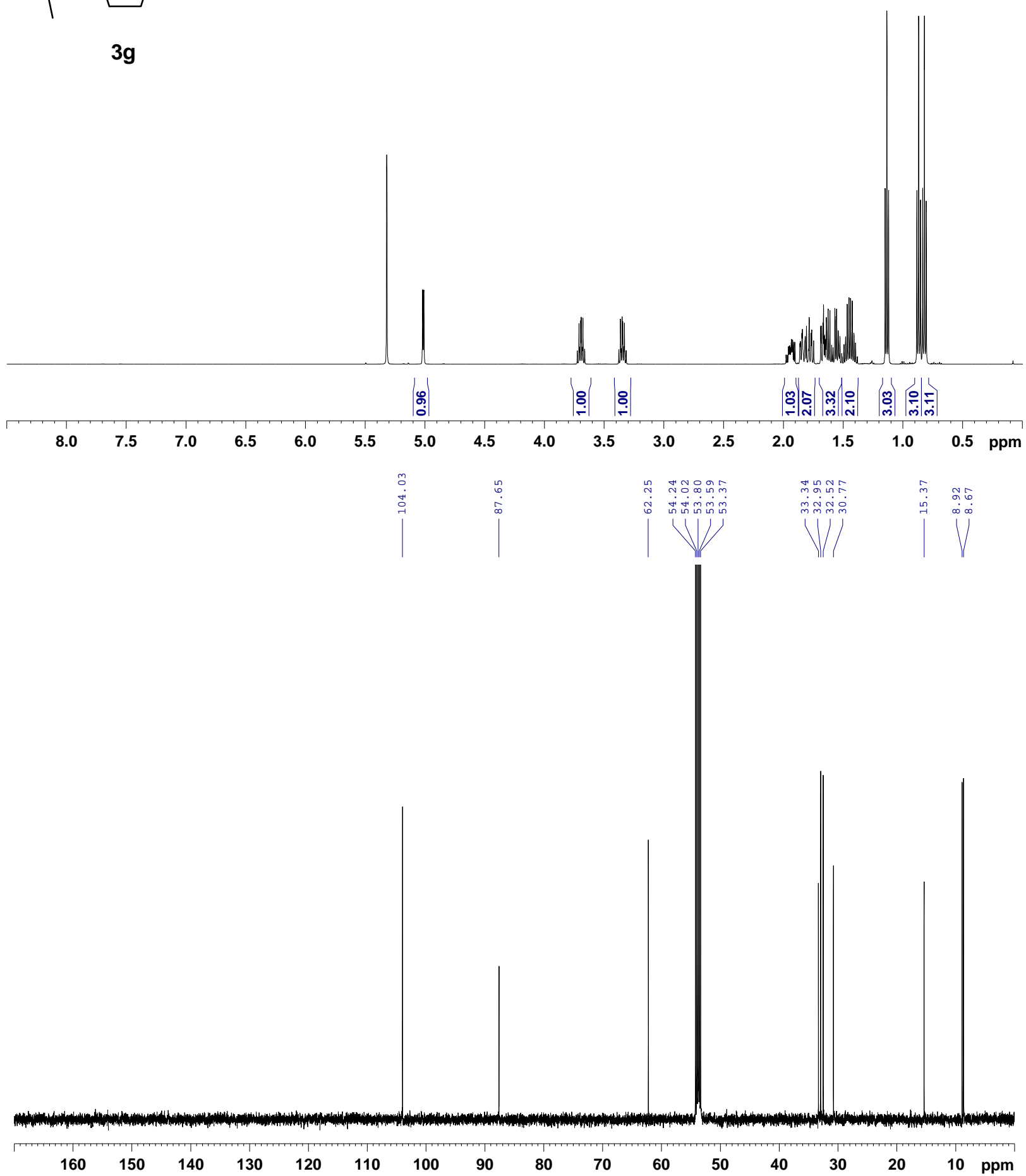


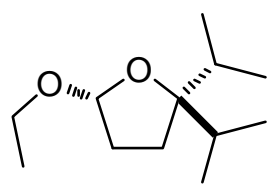

3h
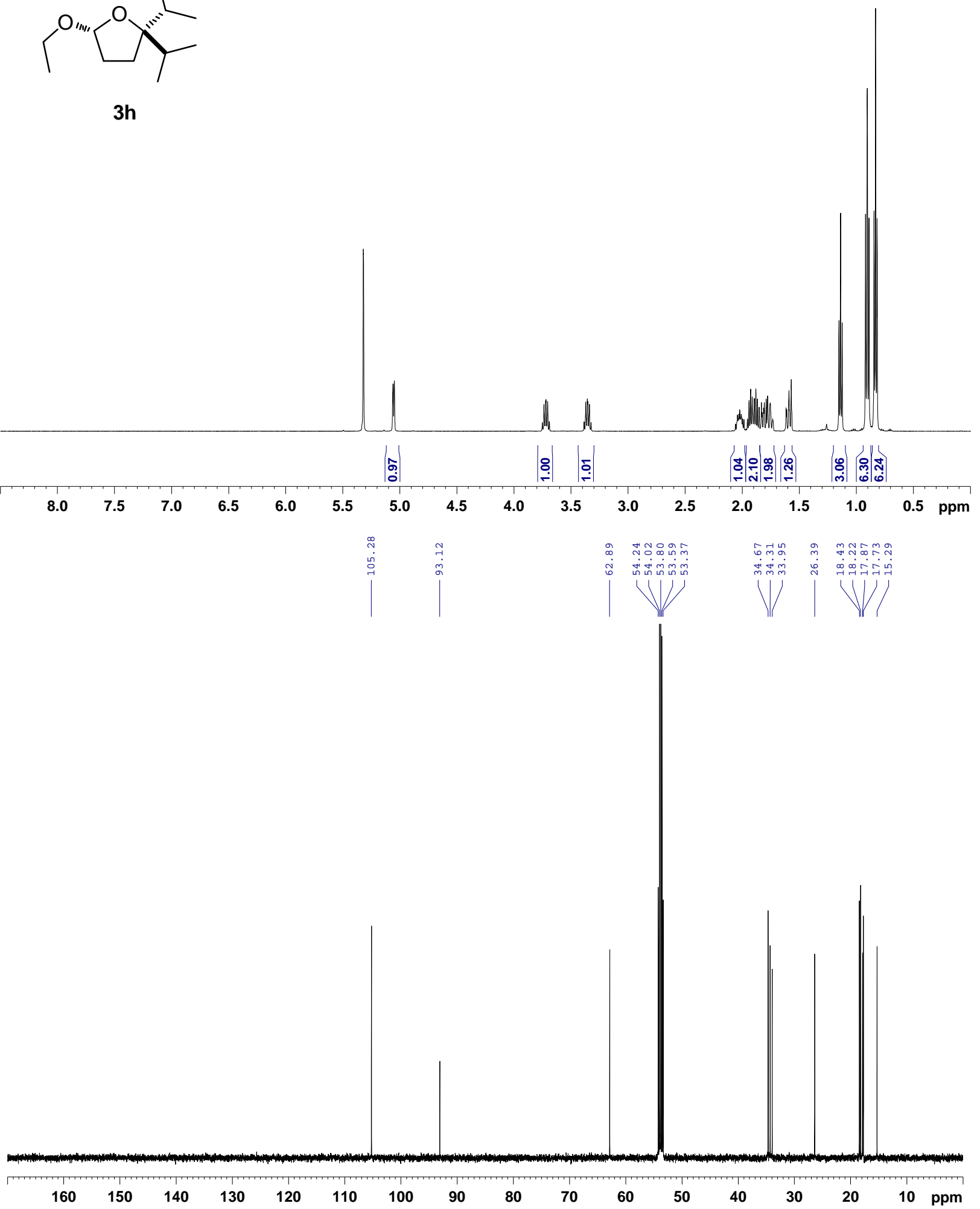


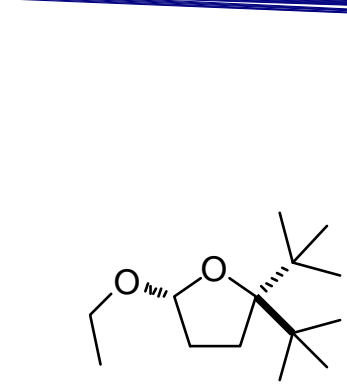

3i
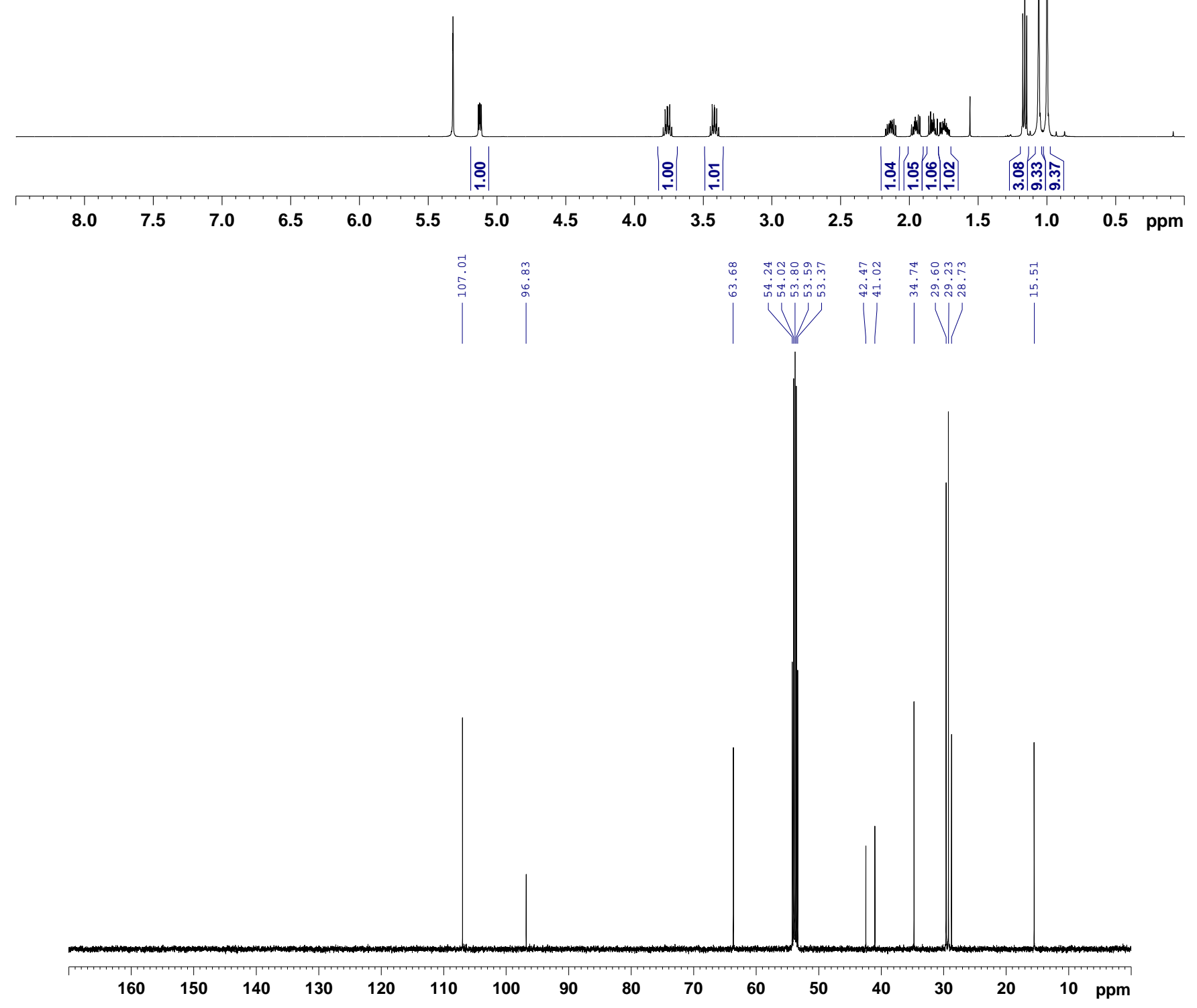


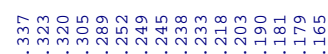

irisinivijizisin

- in<smiles>CCO[C@@H]1CCC(Cc2ccccc2)(Cc2ccccc2)O1</smiles>

3j

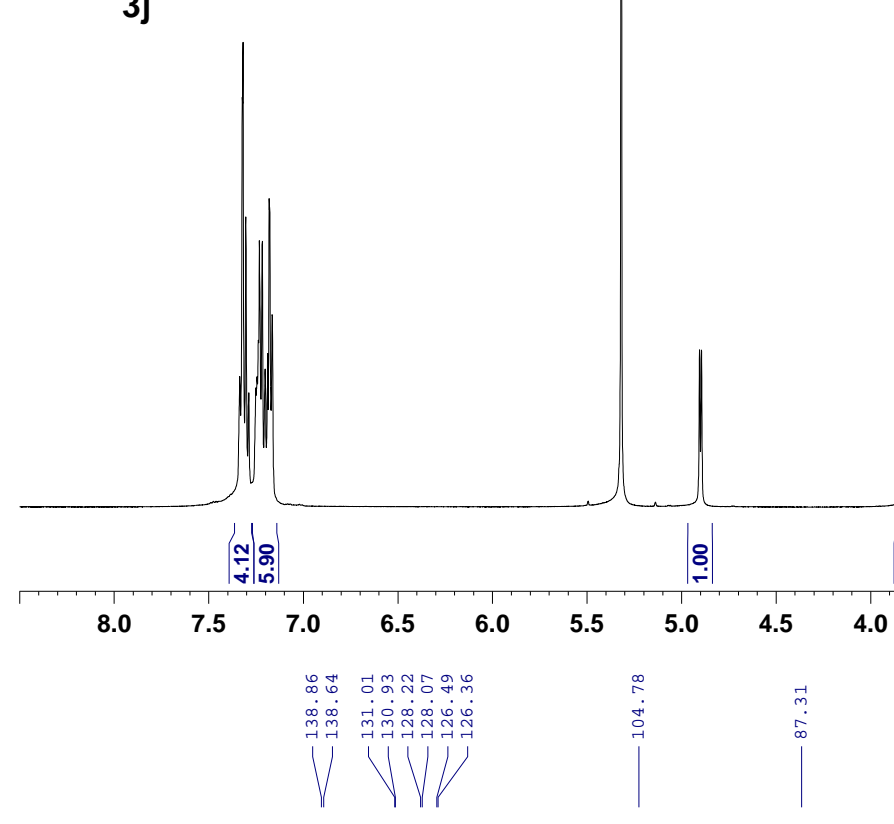

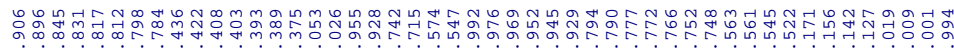

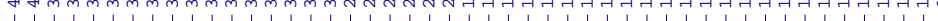

an 
<smiles>CCO[C@@H]1CCC2(CCCCC2)O1</smiles>

3k

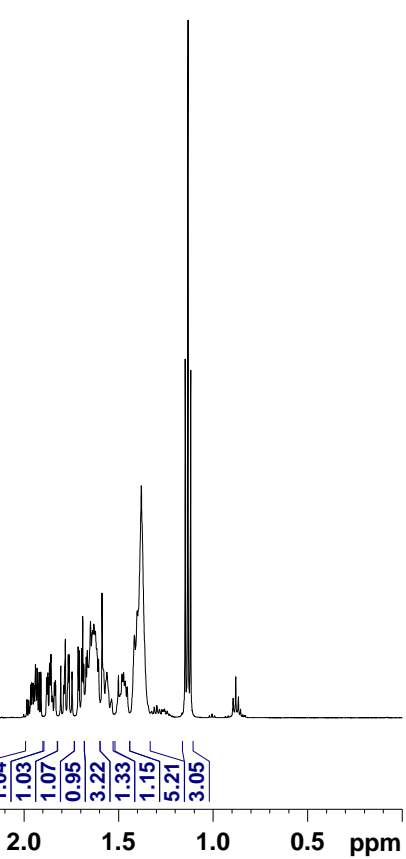

$\begin{array}{llllll}8.0 & 7.5 & 7.0 & 6.5 & 6.0 & 5.5\end{array}$

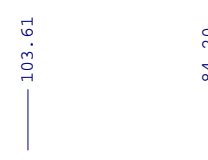

$\stackrel{\stackrel{2}{\infty}}{\stackrel{\infty}{\infty}}$
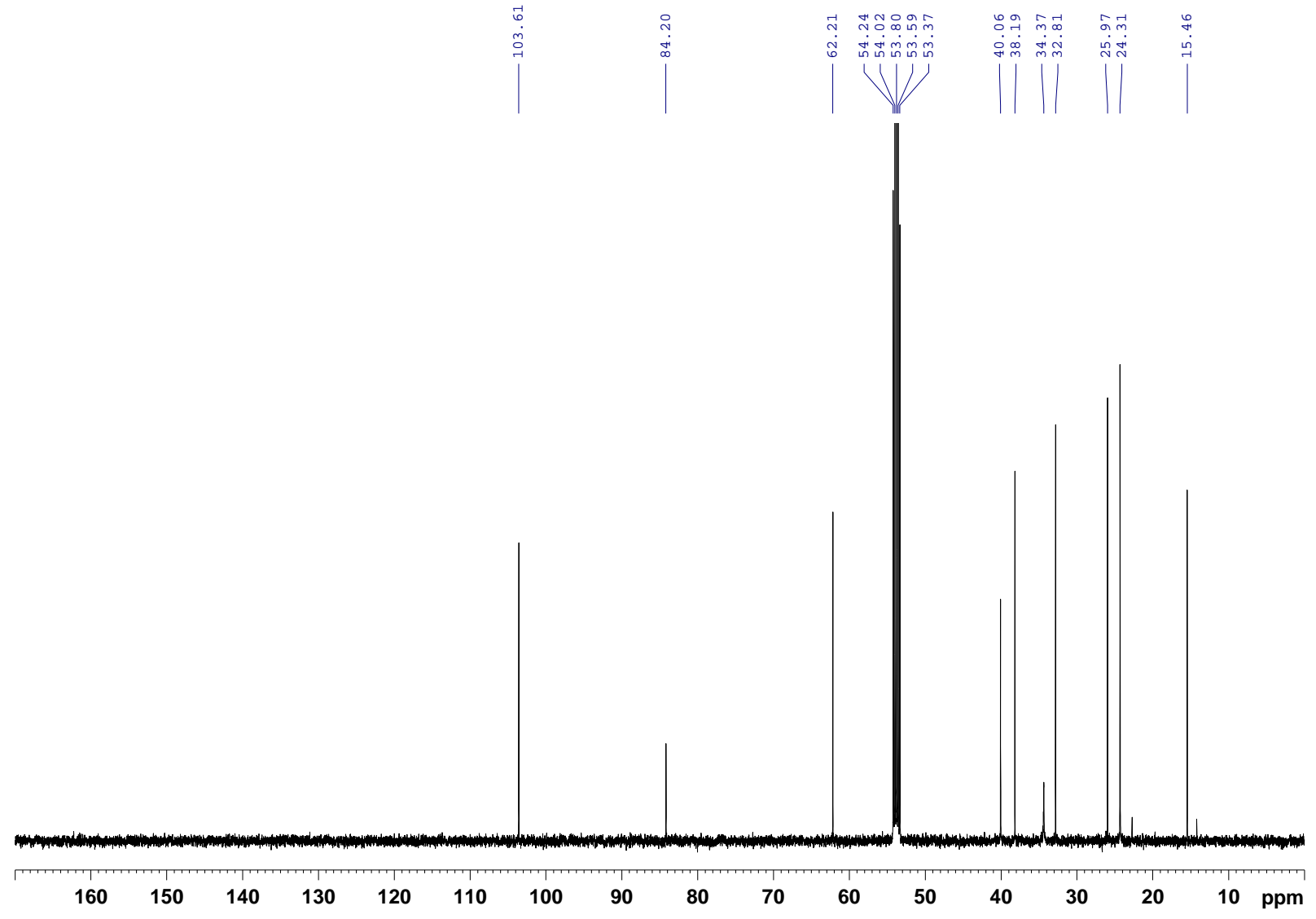
<smiles>CCO[C@H]1CC[C@]2(CCc3ccccc32)O1</smiles>

31

$\mathrm{dr}=1.1: 1$

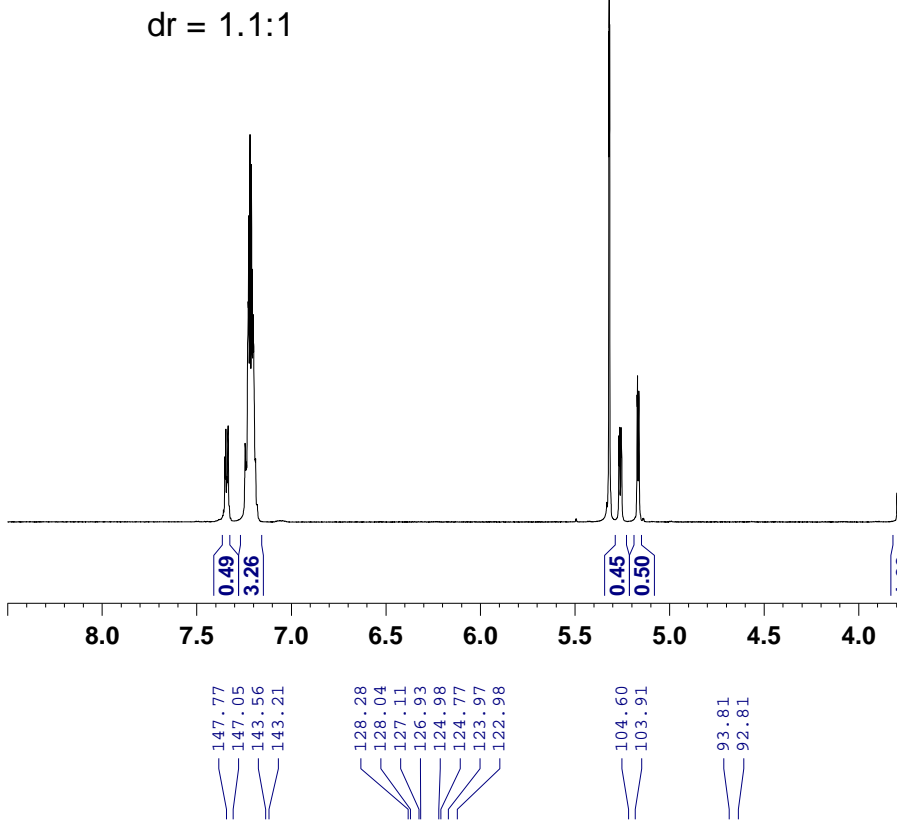

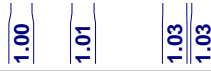

Shl

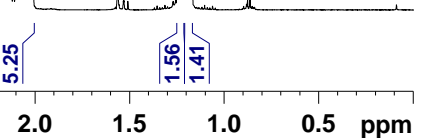

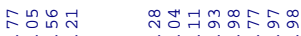

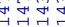

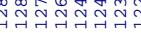

M/Y

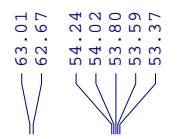

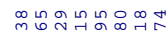

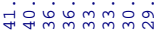

VVVI

웅

붐
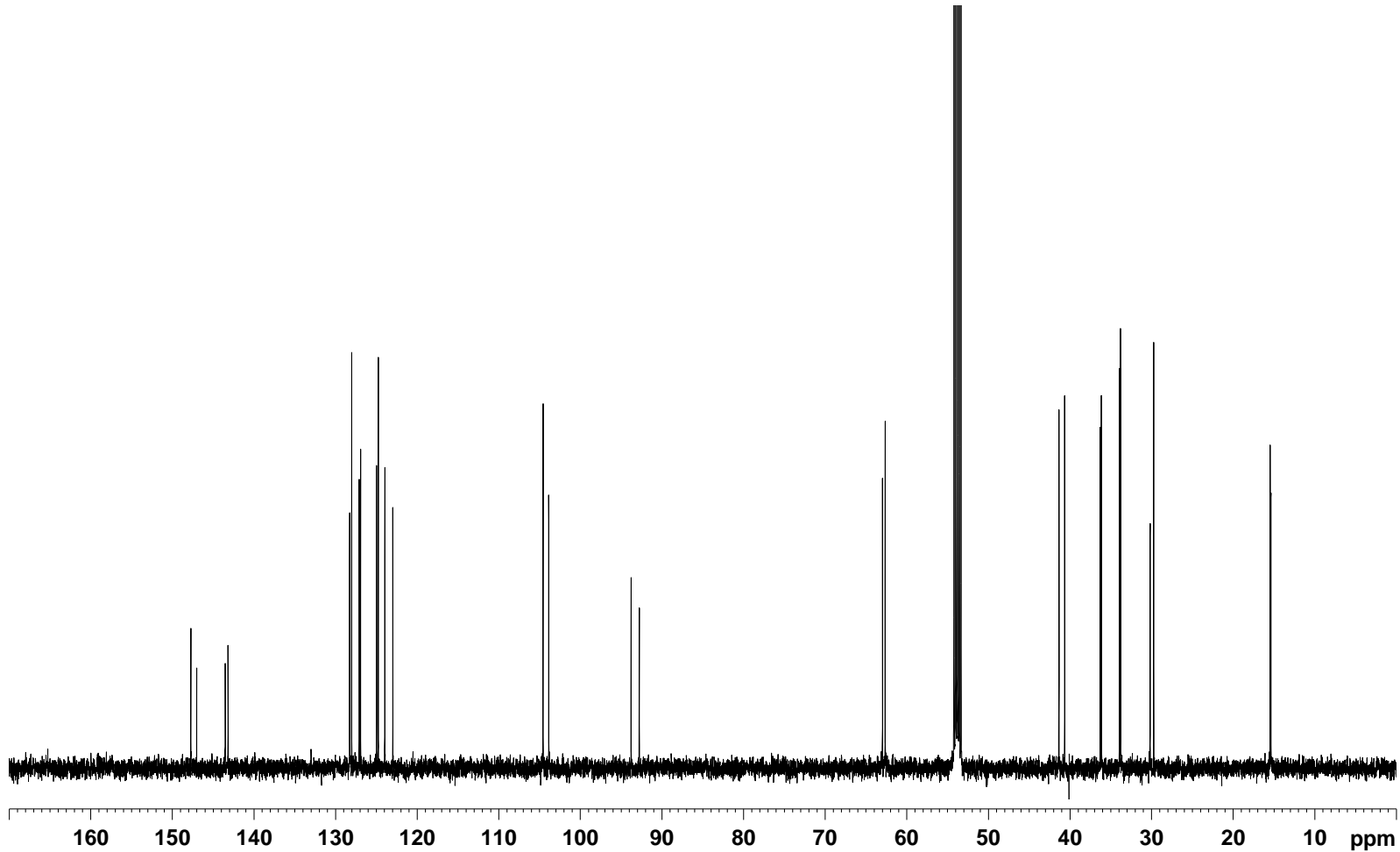


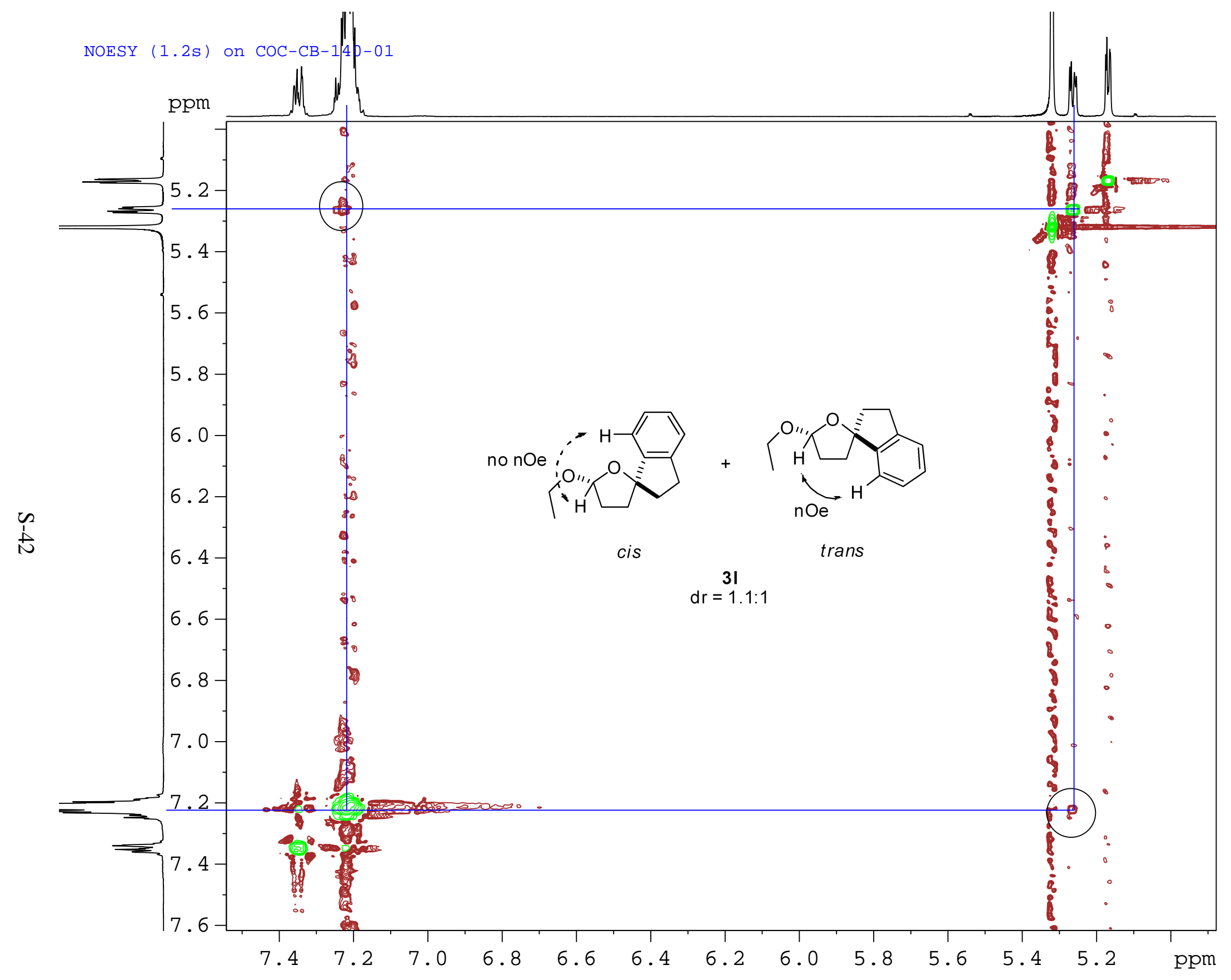

Current Data Parameters

F2 - Acquisition Parameters

Date

20100219
16.34

INSTRUM

$5 \mathrm{~mm}$ PABBO BB-

noesygpph

SOLVENT

2048
$\mathrm{CD} 2 \mathrm{Cl} 2$

NS

$\begin{array}{lr}\text { DS } & 16 \\ \text { SWH } & 4401.409 \mathrm{~Hz} \\ \text { FIDRES } & 2.149125 \mathrm{~Hz}\end{array}$

AQ $\quad 0.2327028 \mathrm{sec}$

RW

DE

DQ $\quad 0.000101 .0 \mathrm{~K}$

D1 $\quad 10.10000038 \mathrm{sec}$

D8 $\quad 1.20000005 \mathrm{sec}$

$\begin{array}{ll}\text { D16 } & 0.00020000 \mathrm{sec} \\ \text { INO } & 0.00022720 \mathrm{sec}\end{array}$

$=======$ CHANNEL $f 1$ ========

$\begin{array}{lr}\text { NUC1 } & 1 \mathrm{H} \text { usec } \\ \text { P1 } & 9.50 \text { usec }\end{array}$

$\begin{array}{ll}\text { P2 } & 19.00 \text { usec } \\ P L 1 & -3.00 \mathrm{~dB}\end{array}$

$\begin{array}{lr}\text { PL1 } & -3.00 \mathrm{~dB} \\ \text { SF01 } & 400.1322007 \mathrm{MHz}\end{array}$

$======$ GRADIENT CHANNEL $====$

GPNAM1 SINE.100

SINE.100

$\begin{array}{lr}\text { GPZ1 } & 40.00 \% \\ \text { GPZ2 } & -40.00 \% \\ \text { P16 } & 1000.00 \%\end{array}$

F1 - Acquisition parameters

$\begin{array}{lc}\text { NDO } & 1 \\ \text { TD } & 2048 \\ \text { SF01 } & \end{array}$

$\begin{array}{lr}\text { SF01 } & 2048 \\ \text { FIDRES } & 400.1322 \mathrm{MHz}\end{array}$

$\begin{array}{lr}2.149148 \mathrm{~Hz} \\ \text { SW } & 11.000 \mathrm{ppm}\end{array}$

FnMODE States-TPPI

F2 - Processing parameters

$\begin{array}{lr}\text { SI } & 4096 \\ \text { SF } & 400.1300150 \mathrm{MHZ}\end{array}$

WDW $\quad 400.1300150$

$\begin{array}{lr}\text { SSB } & 2 \\ \mathrm{LB} & 0.00 \mathrm{~Hz}\end{array}$

$\mathrm{PC}$

F1 - Processing parameters

SI $\quad 4096$

$\begin{array}{ll}\text { MC2 } & \text { States-TPPI } \\ \text { SF } & 400.1300150 \mathrm{MHZ}\end{array}$

WDW $\quad$ QSINE

LB

$\odot . \odot \mathrm{Hz}$ 

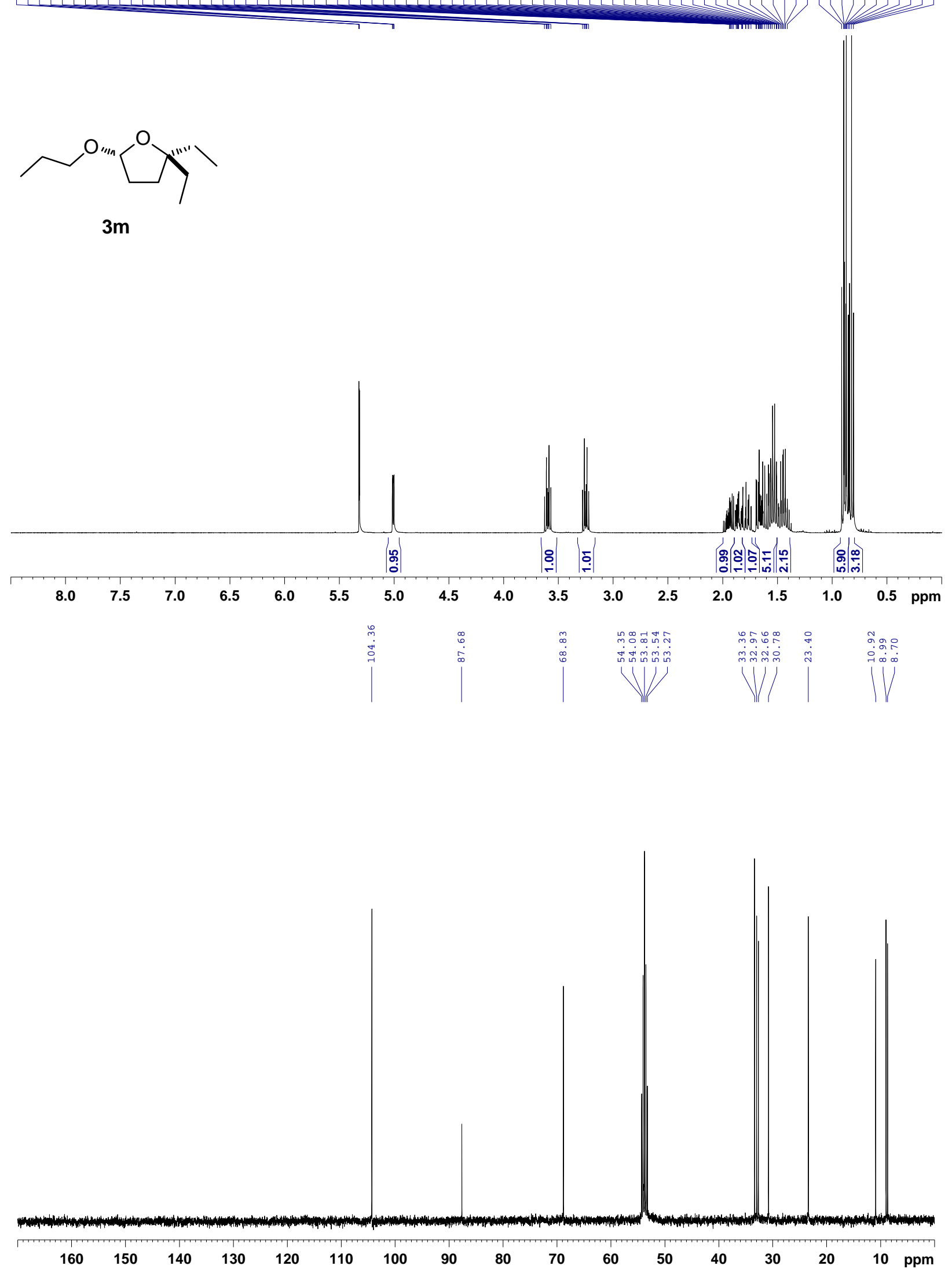


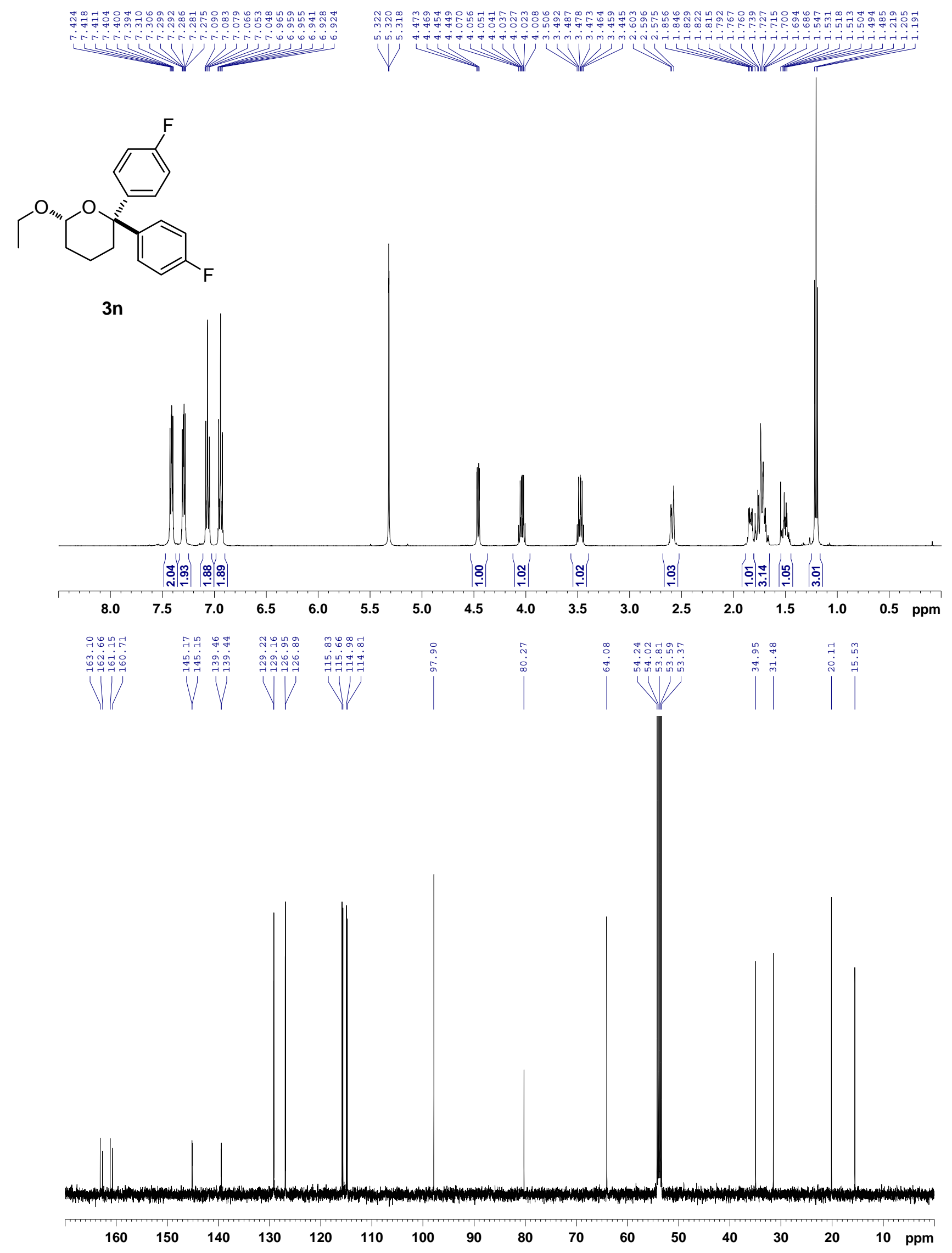




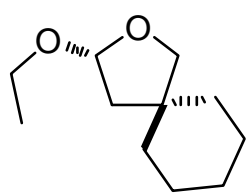

30
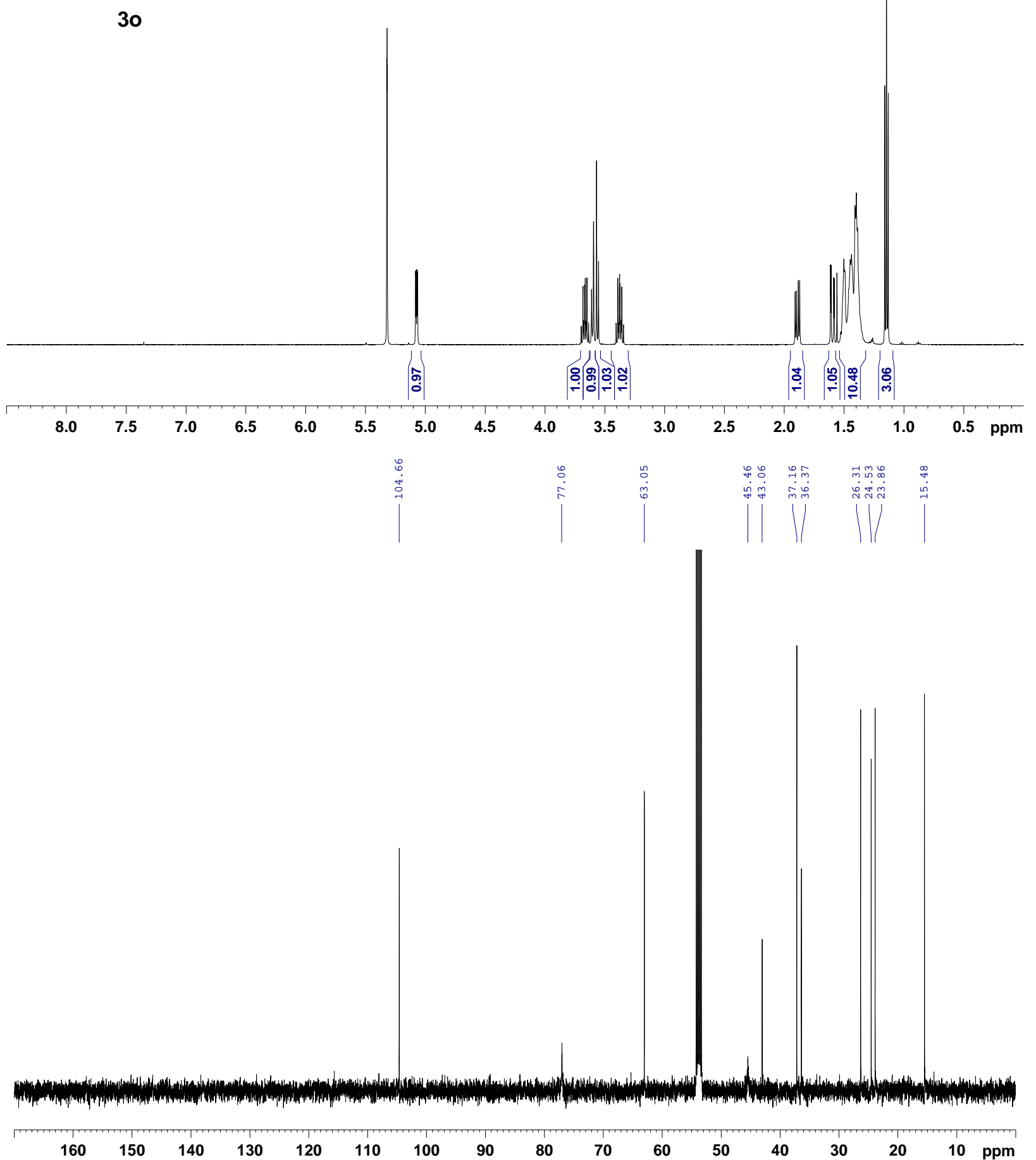

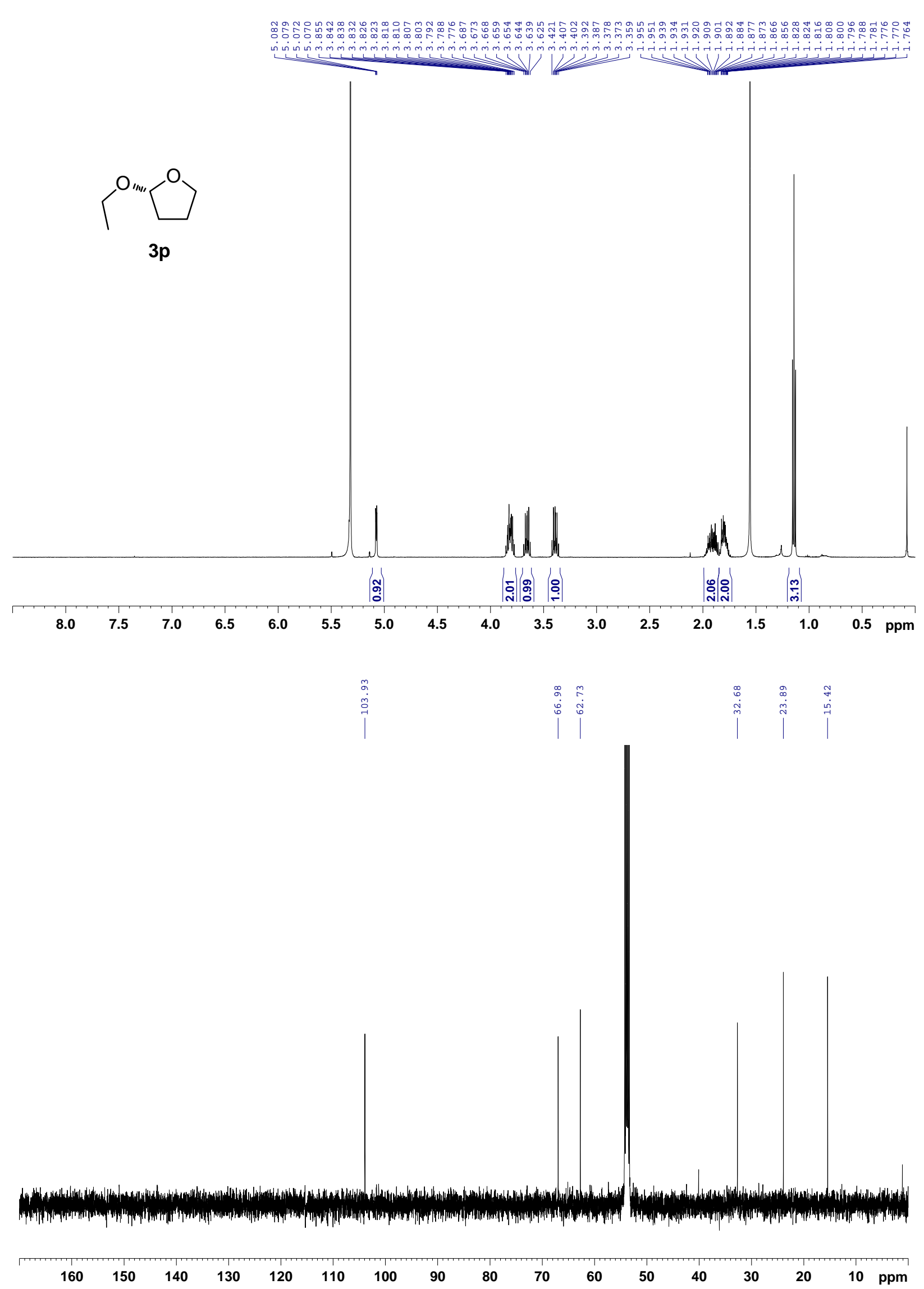


\section{HPLC and GC Traces}

mAU

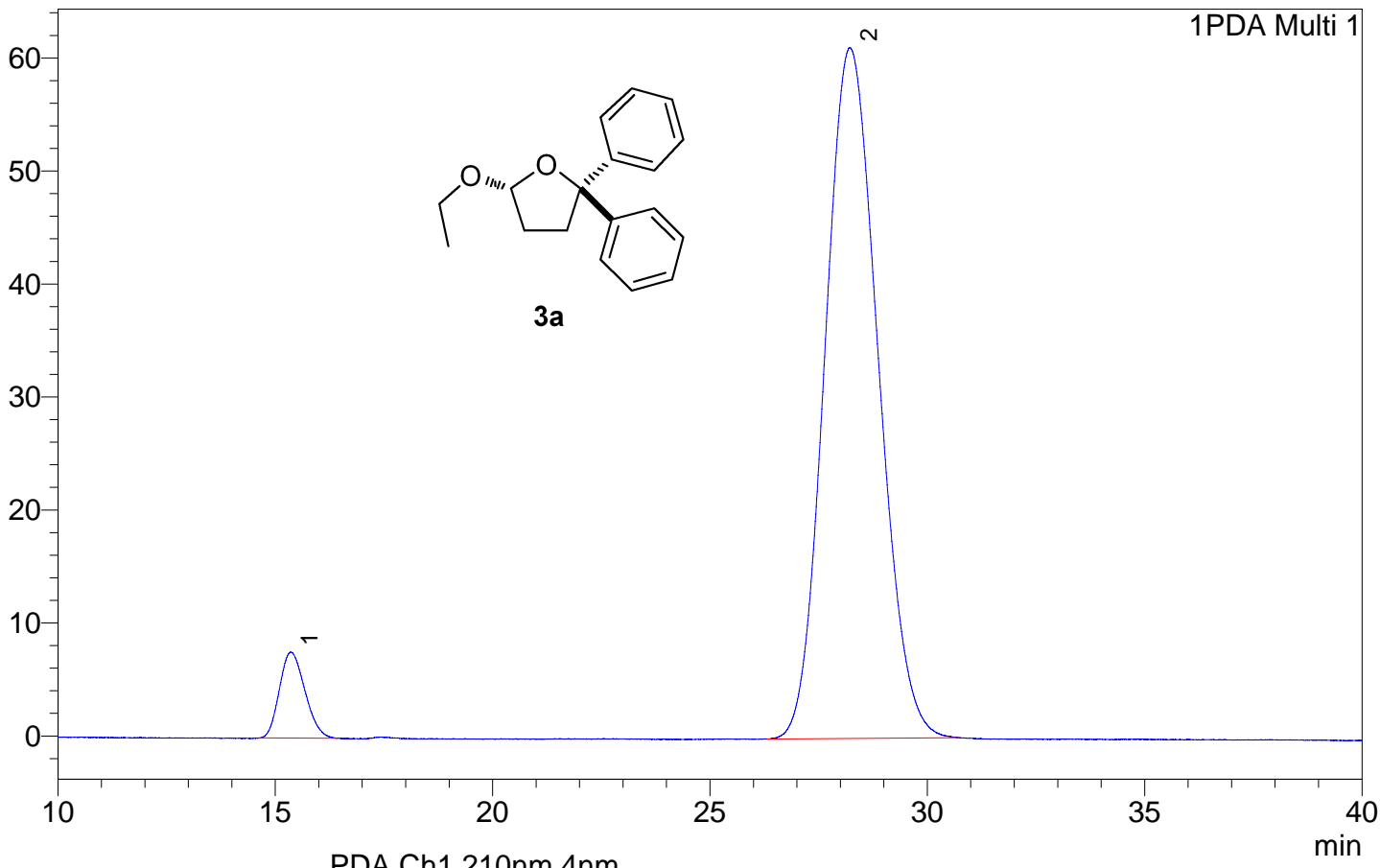

$1210 \mathrm{~nm} 4 \mathrm{~nm}$

\begin{tabular}{|c|c|c|}
\hline Peak \# & Ret. Time & Area \% \\
\hline 1 & 15.35 & 5.63 \\
\hline 2 & 28.21 & 94.37 \\
\hline Total & & 100.00 \\
\hline
\end{tabular}

mAU

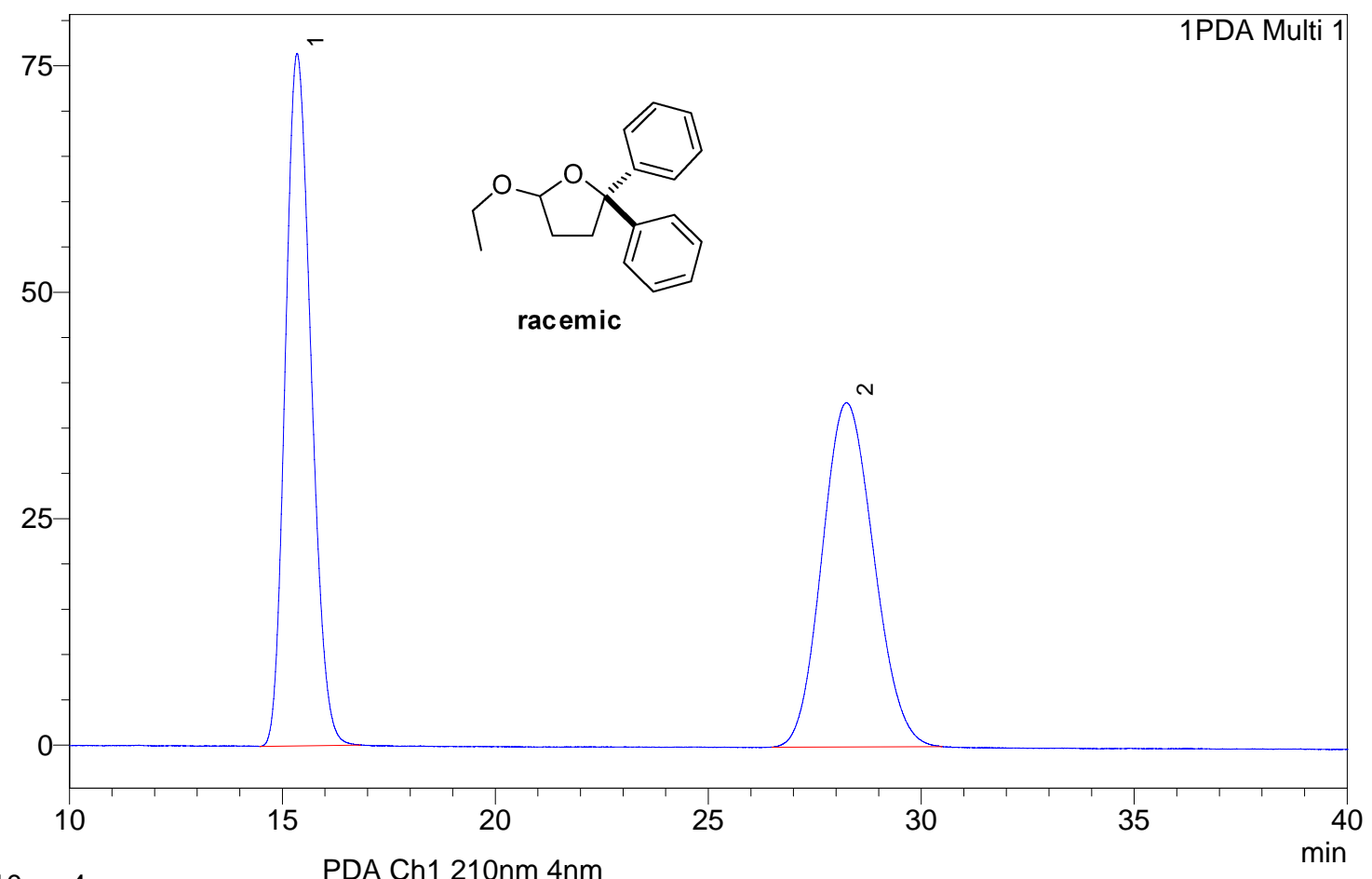

$1210 \mathrm{~nm} 4 \mathrm{~nm}$

\begin{tabular}{|c|c|c|}
\hline Peak \# & Ret. Time & Area \% \\
\hline 1 & 15.34 & 50.05 \\
\hline 2 & 28.24 & 49.95 \\
\hline Total & & 100.00 \\
\hline
\end{tabular}


mAU

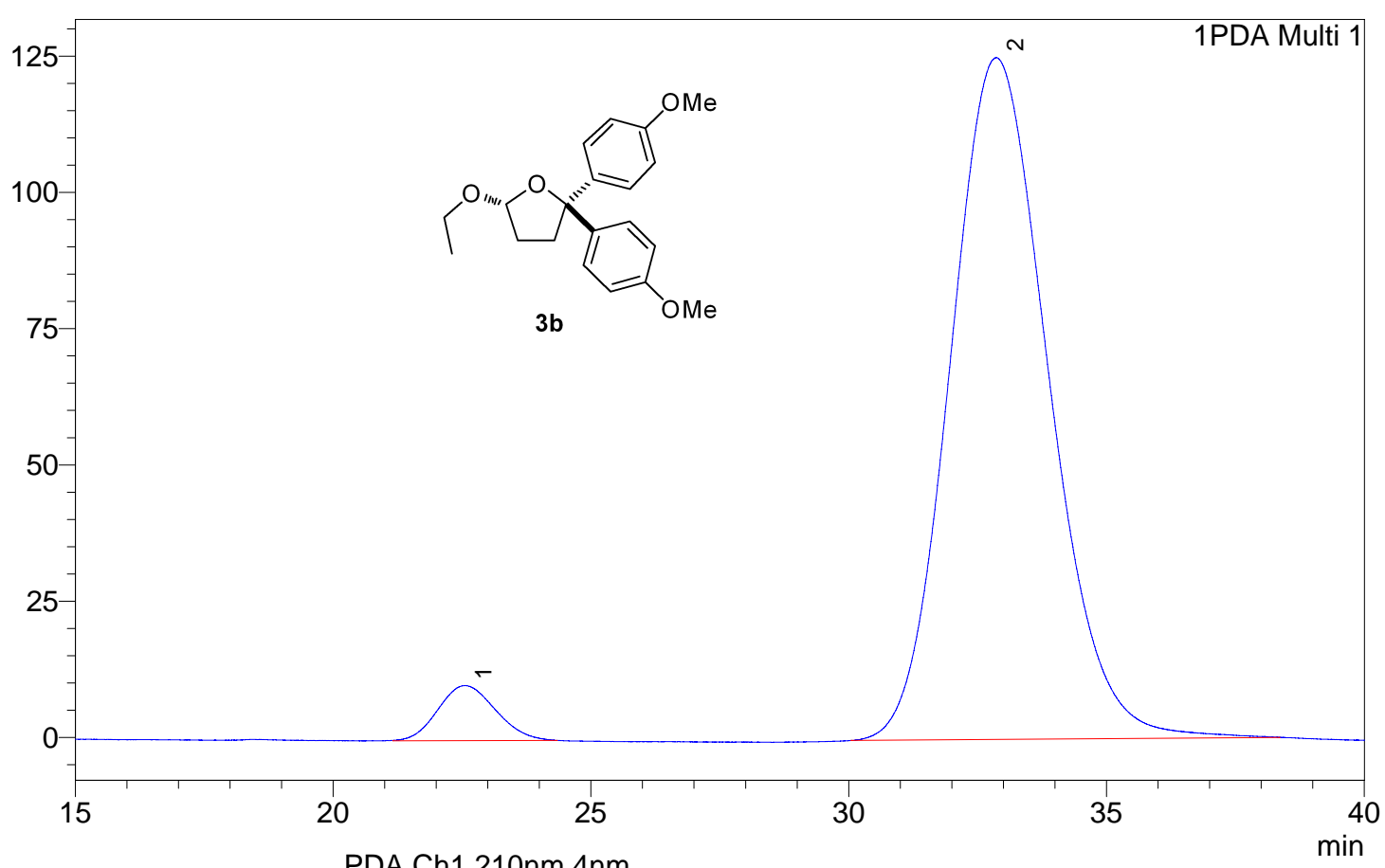

$1210 \mathrm{~nm} 4 \mathrm{~nm}$

\begin{tabular}{|c|c|c|}
\hline Peak \# & Ret. Time & Area \% \\
\hline 1 & 22.55 & 4.53 \\
\hline 2 & 32.86 & 95.47 \\
\hline Total & & 100.00 \\
\hline
\end{tabular}

mAU

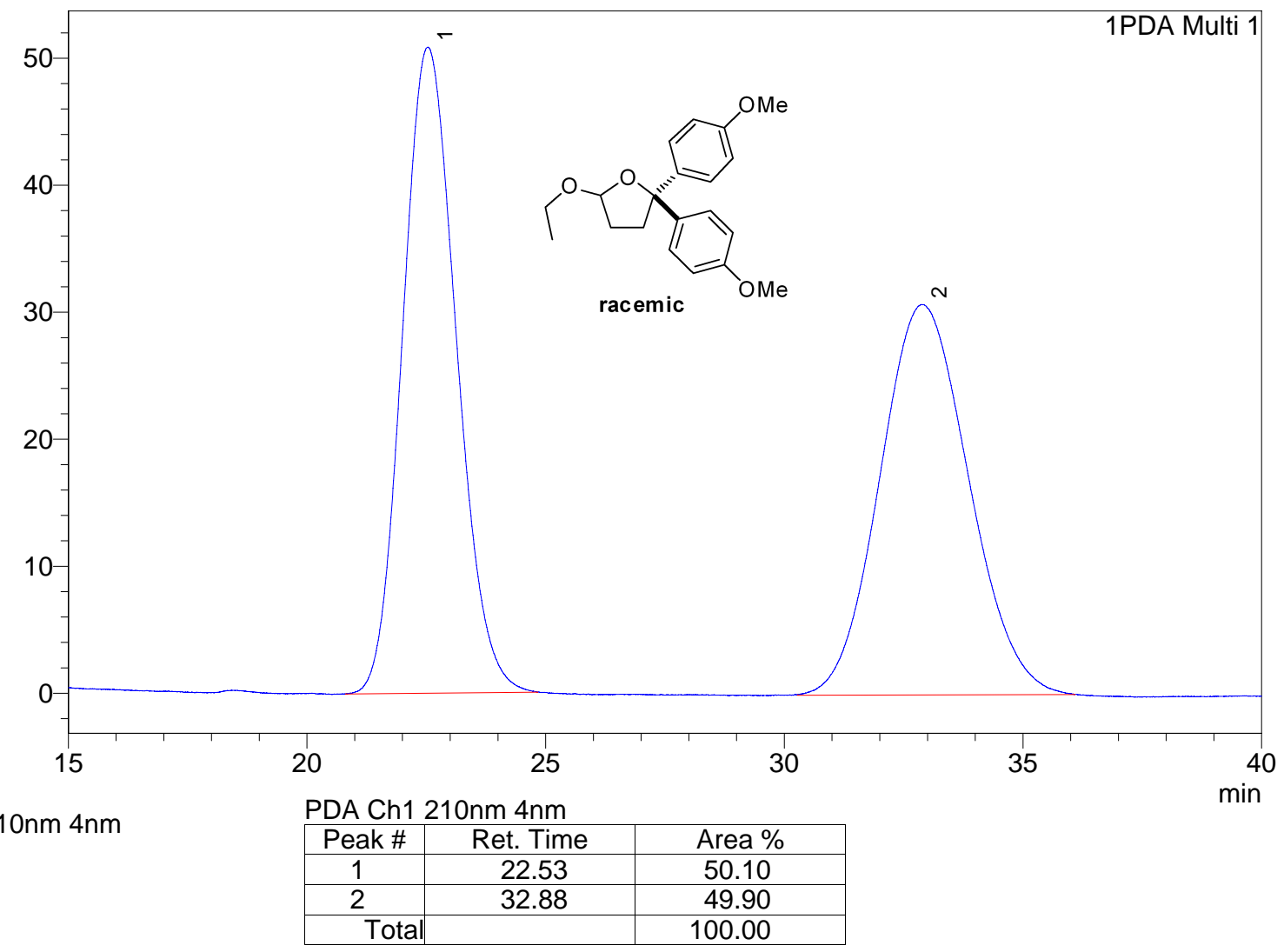


mAU

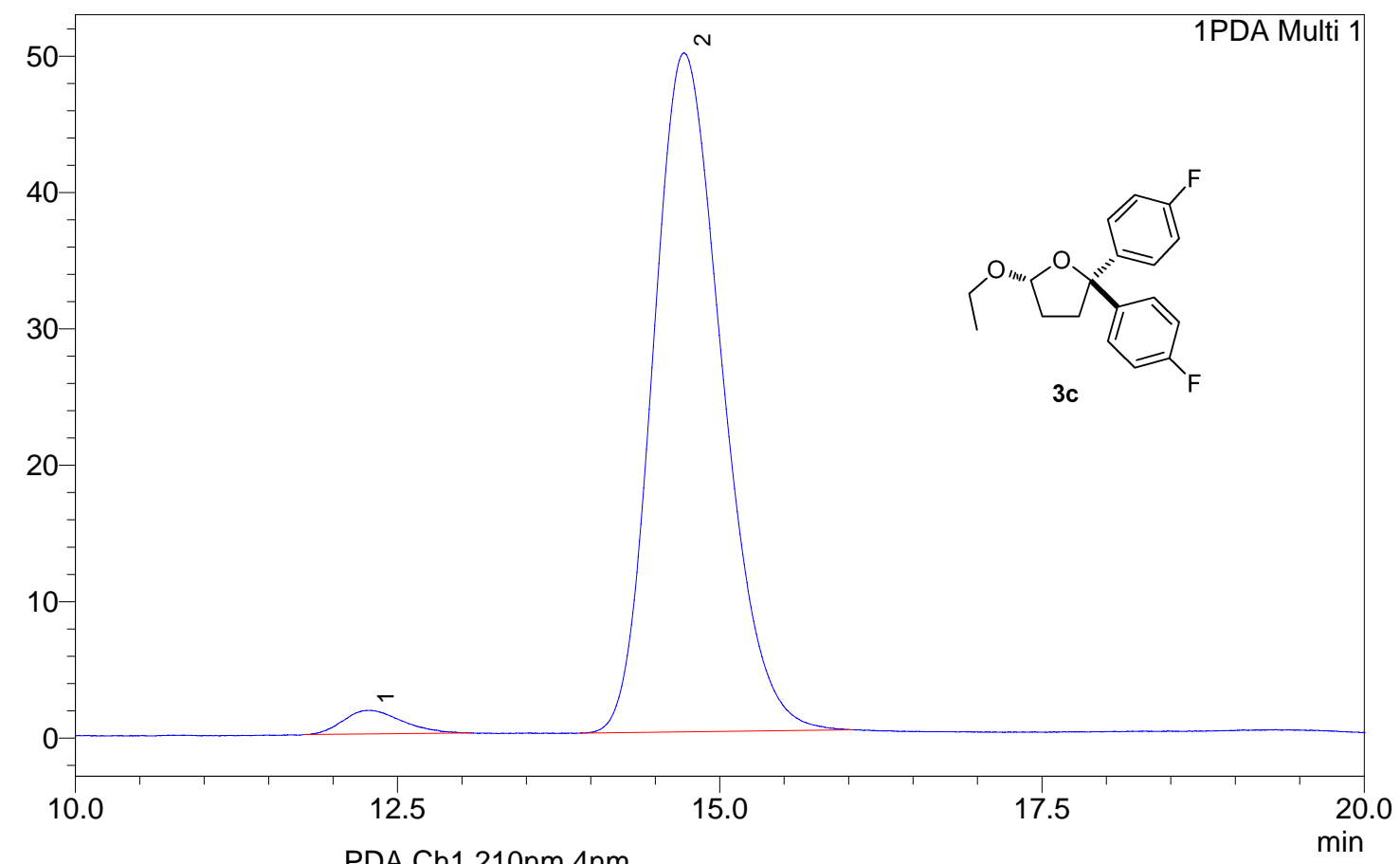

$1210 \mathrm{~nm} 4 \mathrm{~nm}$

PDA Ch1 210nm 4nm

\begin{tabular}{|c|c|c|}
\hline Peak \# & Ret. Time & Area \% \\
\hline 1 & 12.26 & 2.89 \\
\hline 2 & 14.72 & 97.11 \\
\hline Total & & 100.00 \\
\hline
\end{tabular}

mAU

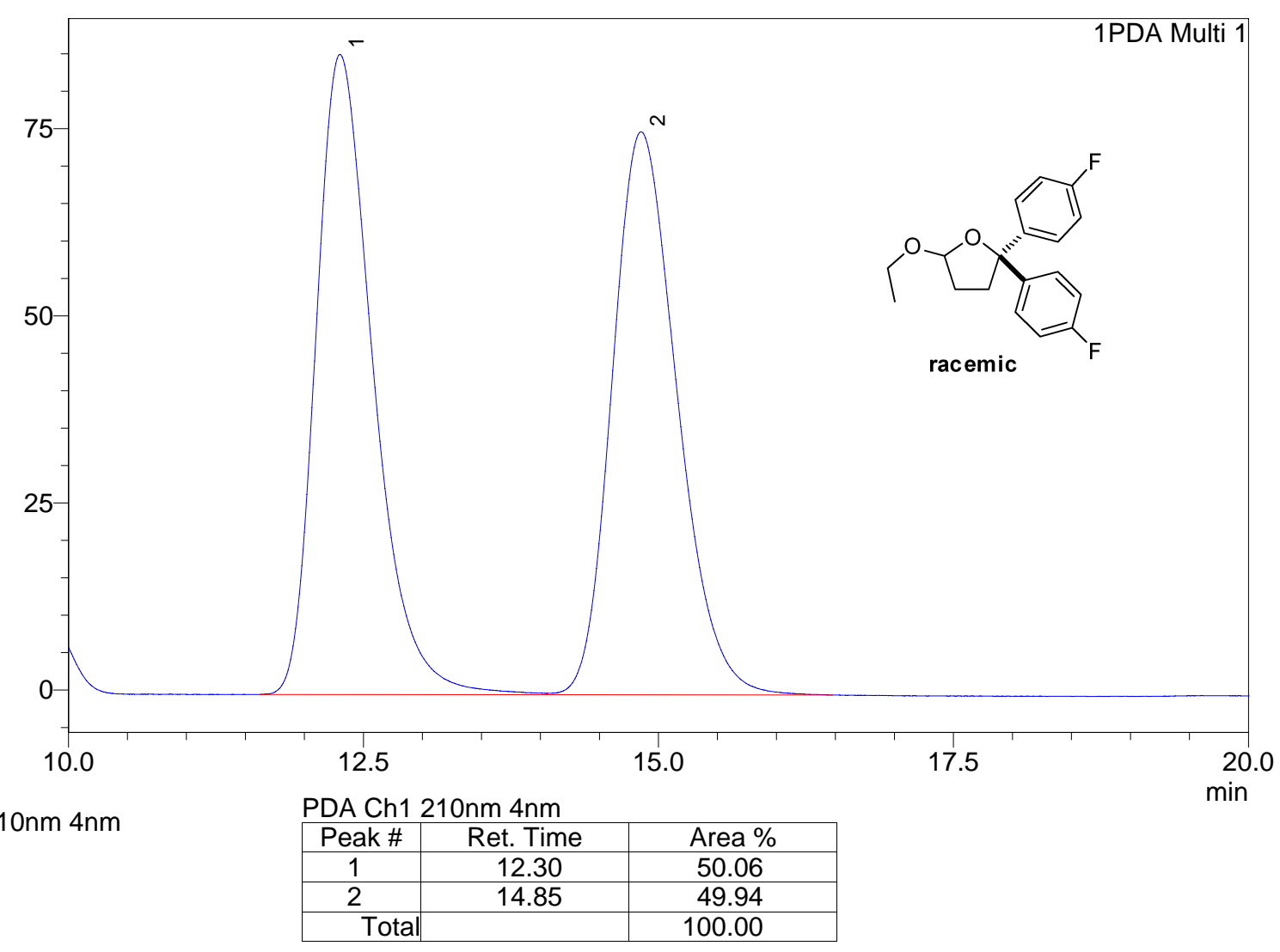


mAU

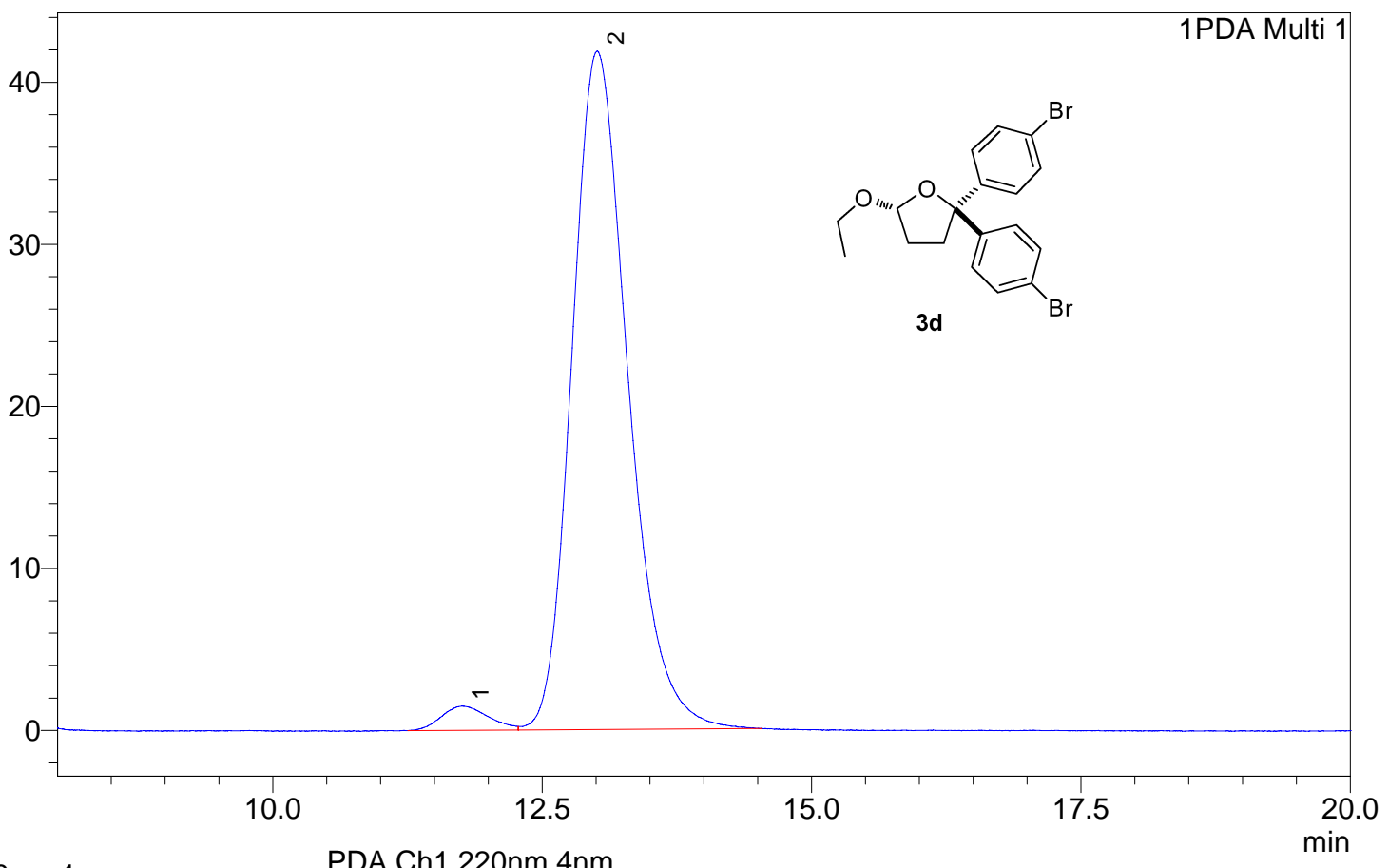

$1220 \mathrm{~nm} 4 \mathrm{~nm}$

\begin{tabular}{|c|c|c|}
\hline Peak \# & Ret. Time & Area \% \\
\hline 1 & 11.75 & 2.92 \\
\hline 2 & 13.01 & 97.08 \\
\hline Total & & 100.00 \\
\hline
\end{tabular}

mAU

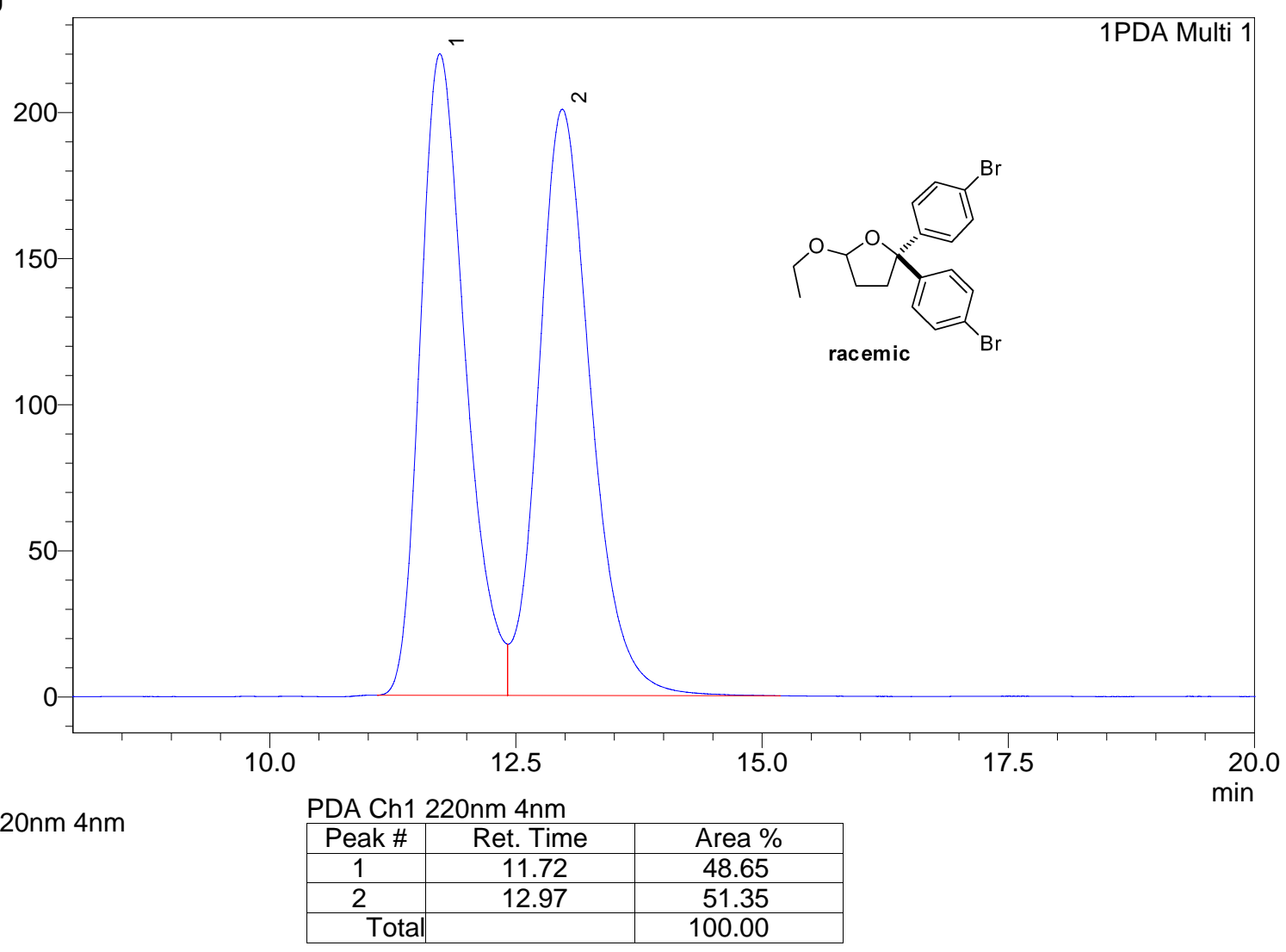



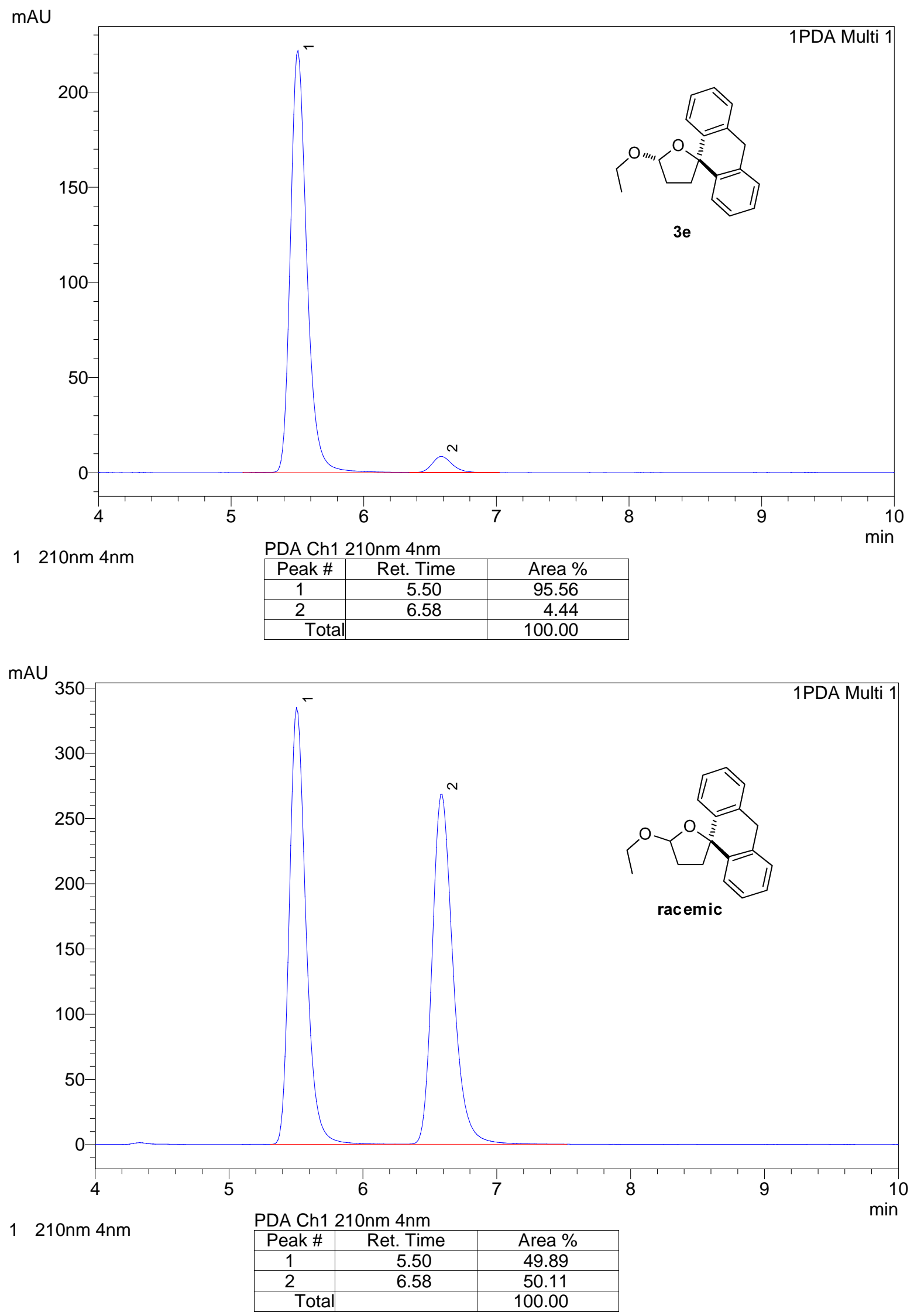

S-51 

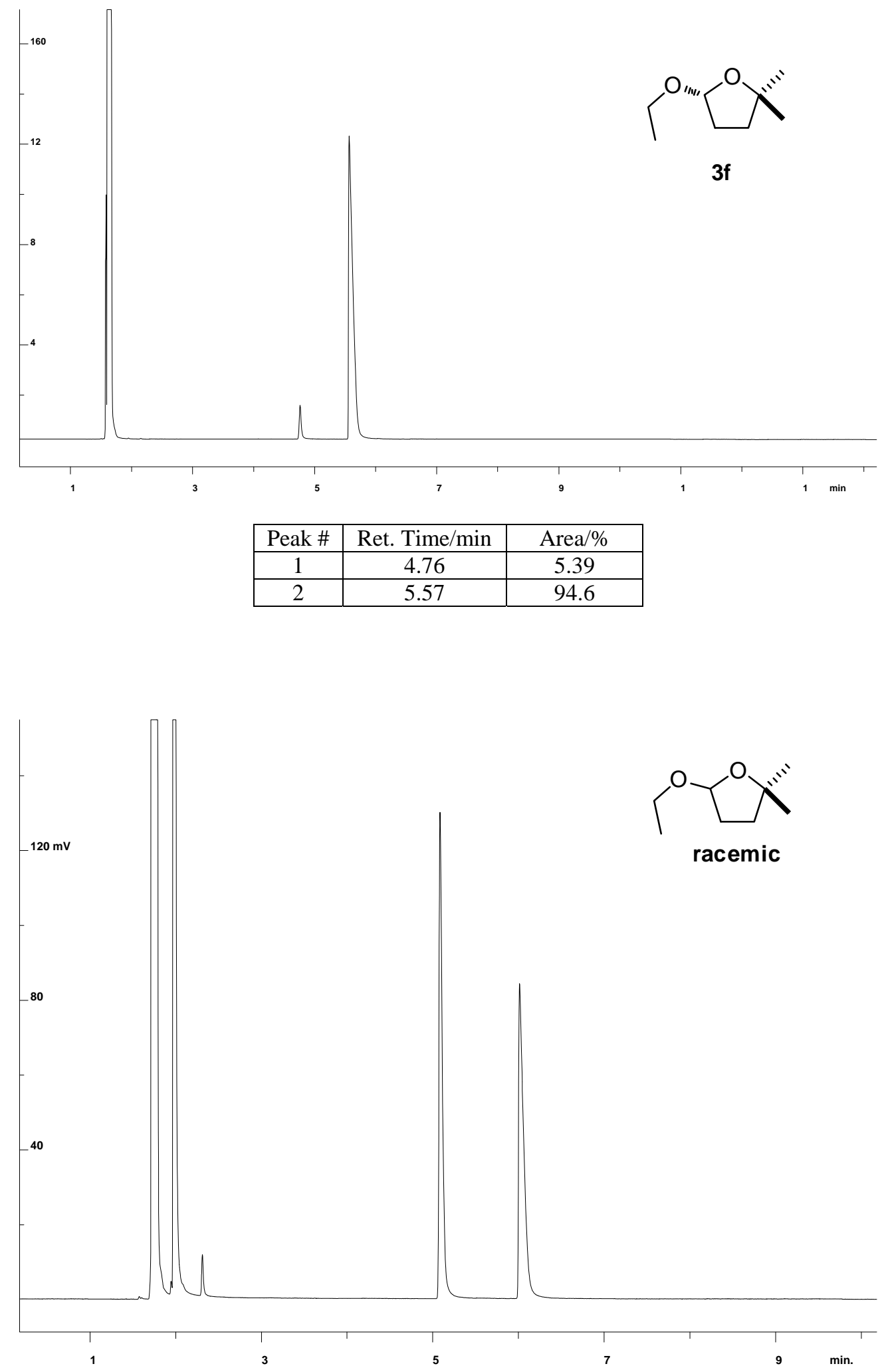

\begin{tabular}{|c|c|c|}
\hline Peak \# & Ret. Time/min & Area/\% \\
\hline 1 & 5.09 & 50.1 \\
\hline 2 & 6.01 & 49.9 \\
\hline
\end{tabular}



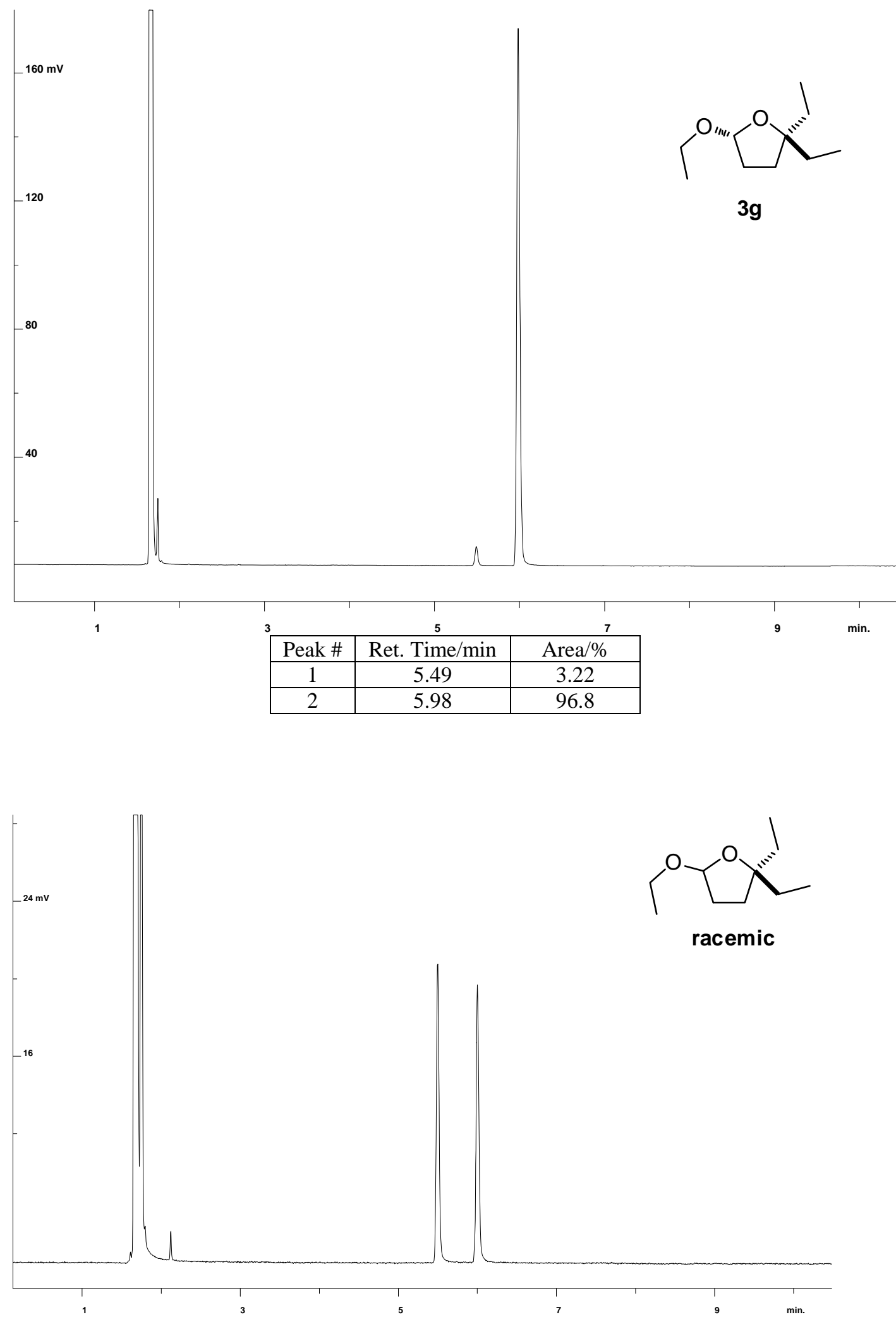

\begin{tabular}{|c|c|c|}
\hline Peak \# & Ret. Time/min & Area/\% \\
\hline 1 & 5.50 & 50.4 \\
\hline 2 & 6.00 & 49.6 \\
\hline
\end{tabular}

S-53 

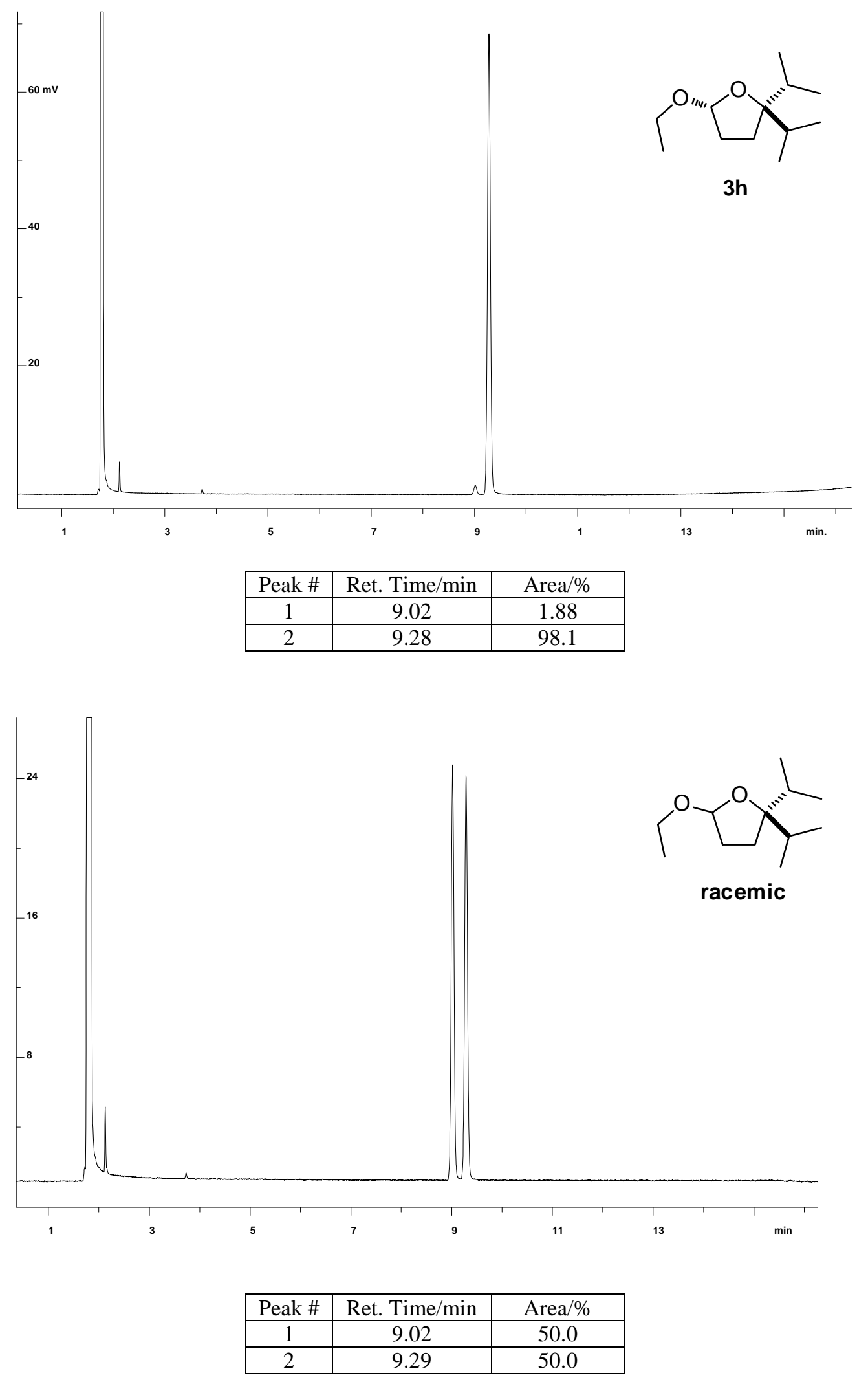

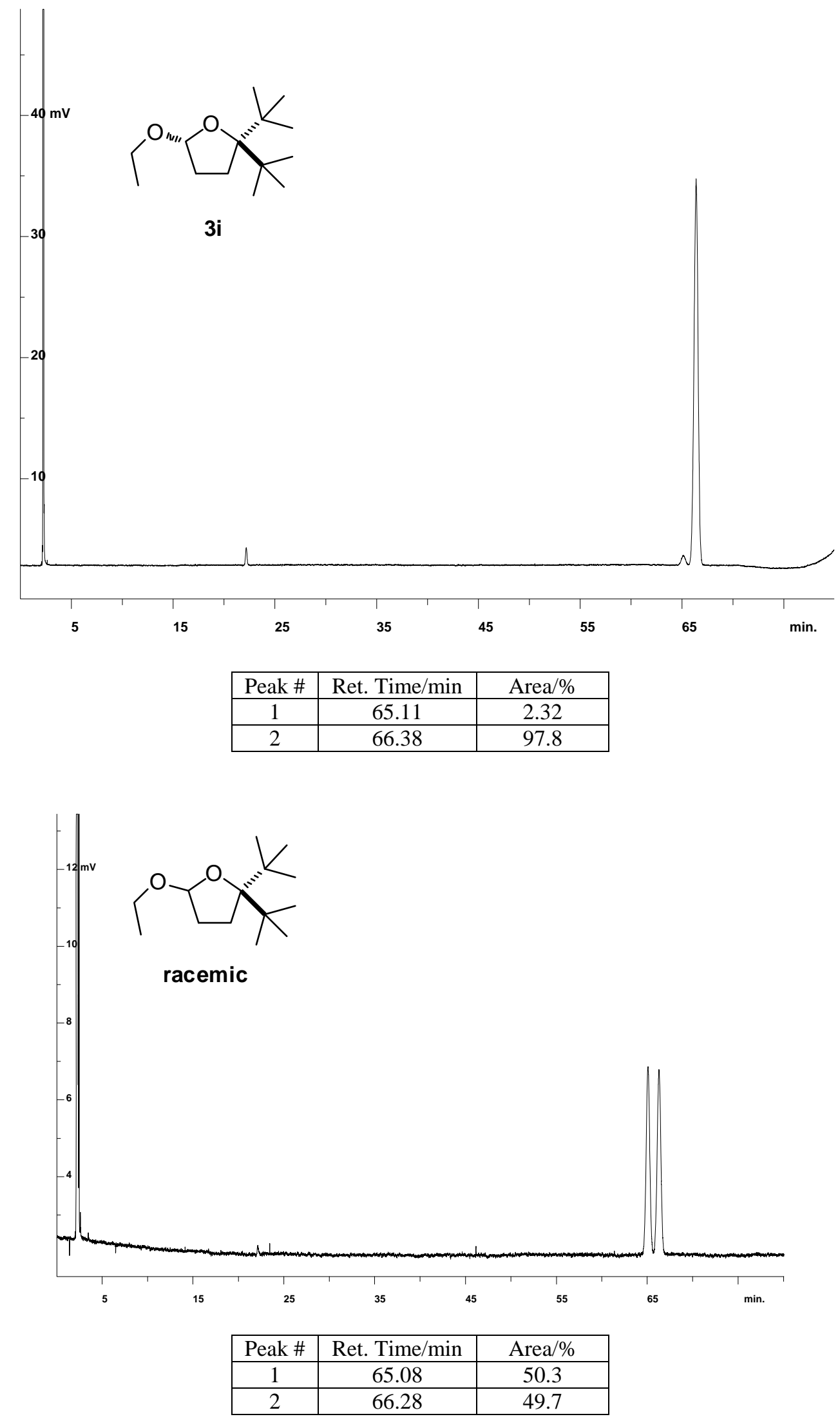

S-55 
mAU

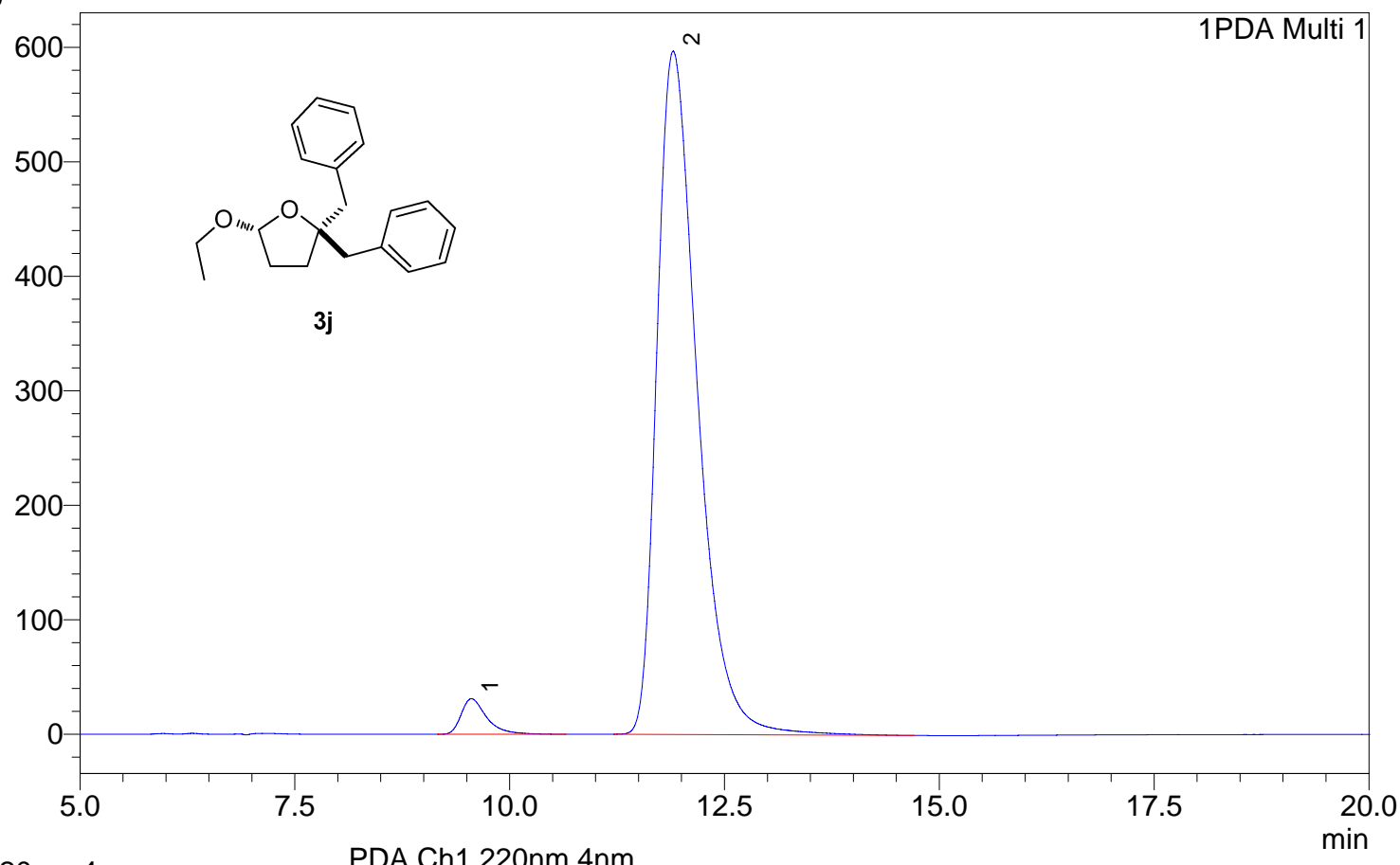

$1220 \mathrm{~nm} 4 \mathrm{~nm}$

\begin{tabular}{|c|c|c|}
\hline Peak \# & Ret. Time & Area \% \\
\hline 1 & 9.55 & 3.18 \\
\hline 2 & 11.90 & 96.82 \\
\hline Total & & 100.00 \\
\hline
\end{tabular}

mAU

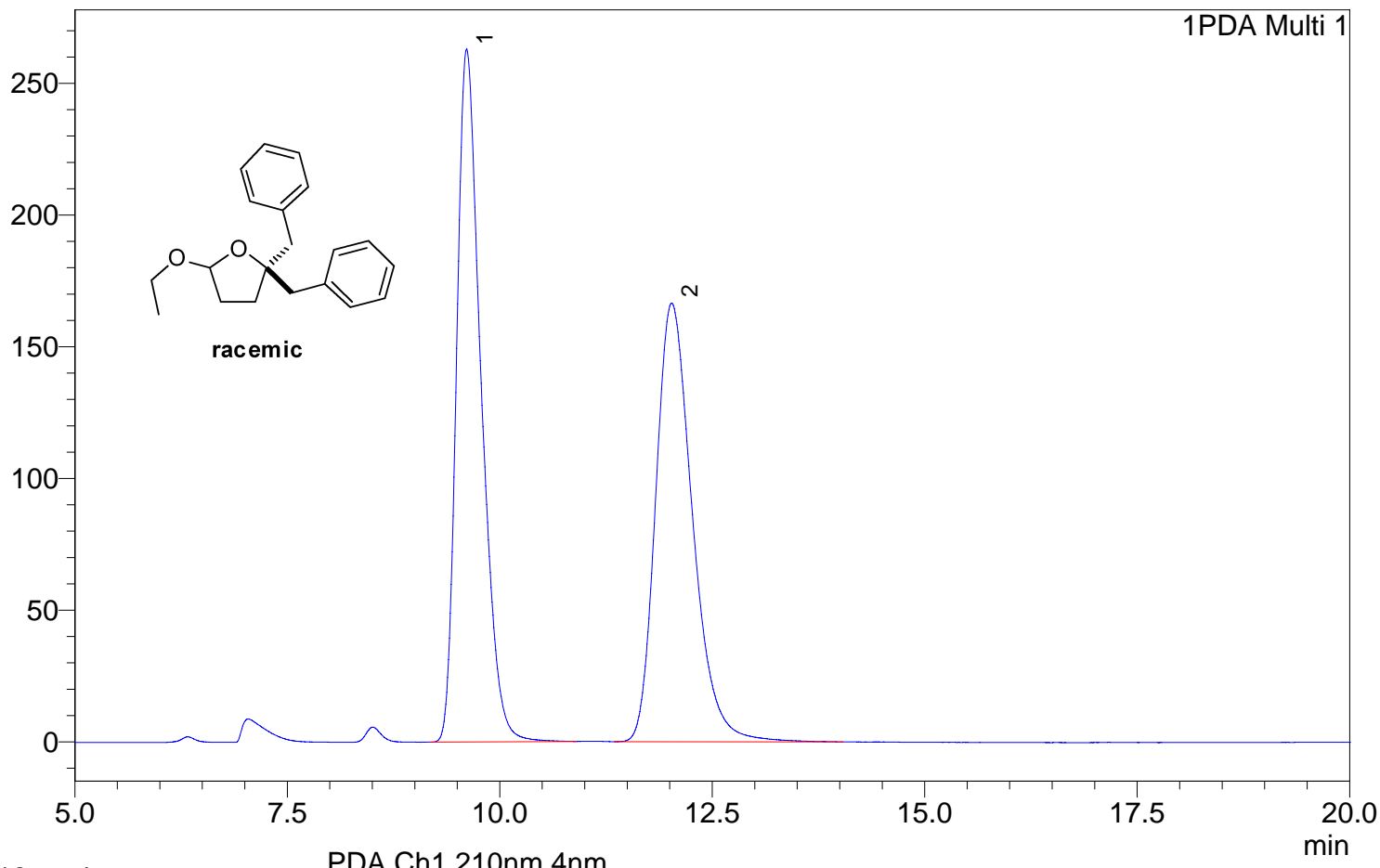

$1210 \mathrm{~nm} 4 \mathrm{~nm}$

\begin{tabular}{|c|c|c|}
\hline Peak \# & Ret. Time & Area \% \\
\hline 1 & 9.60 & 51.33 \\
\hline 2 & 12.02 & 48.67 \\
\hline Total & & 100.00 \\
\hline
\end{tabular}



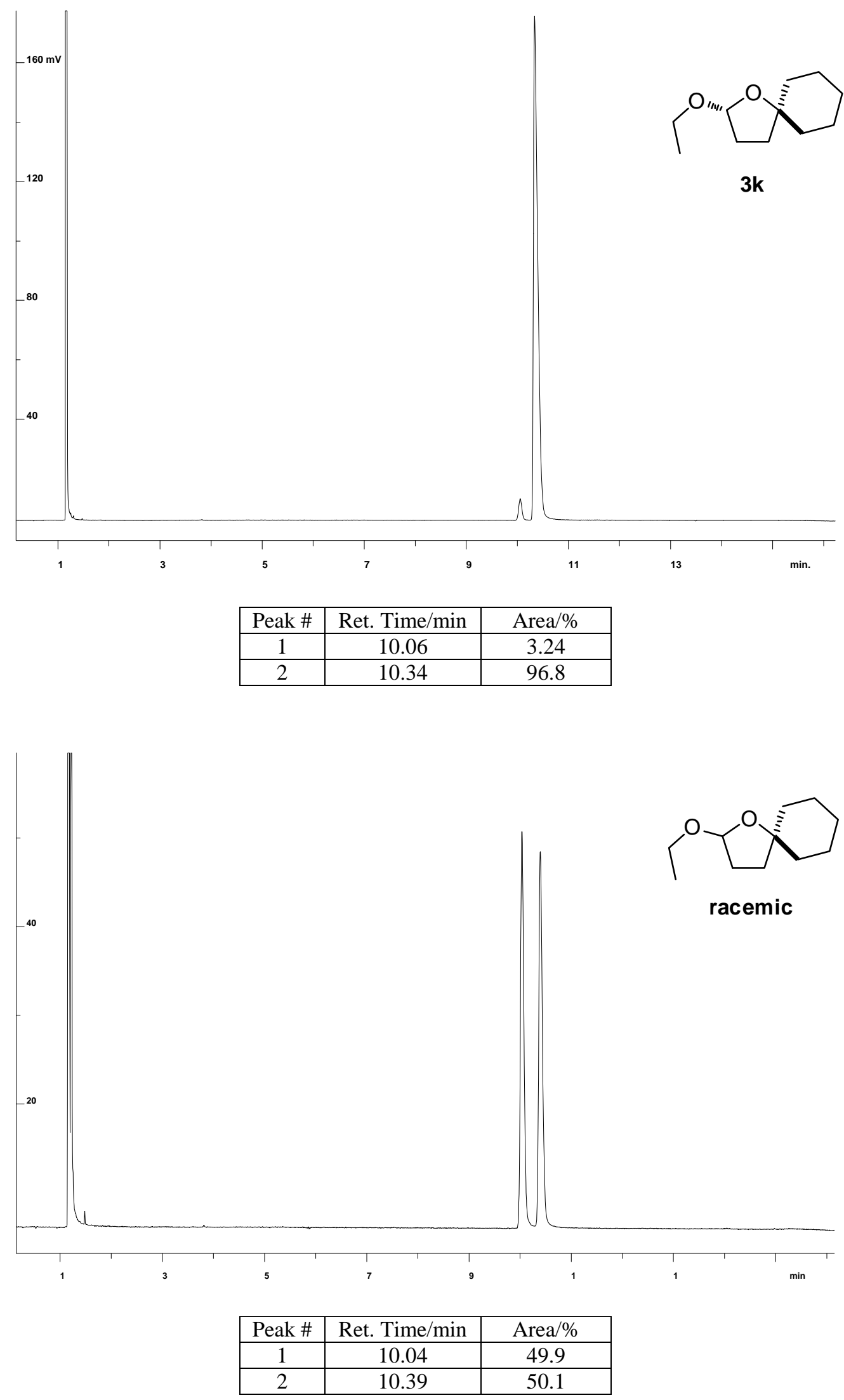

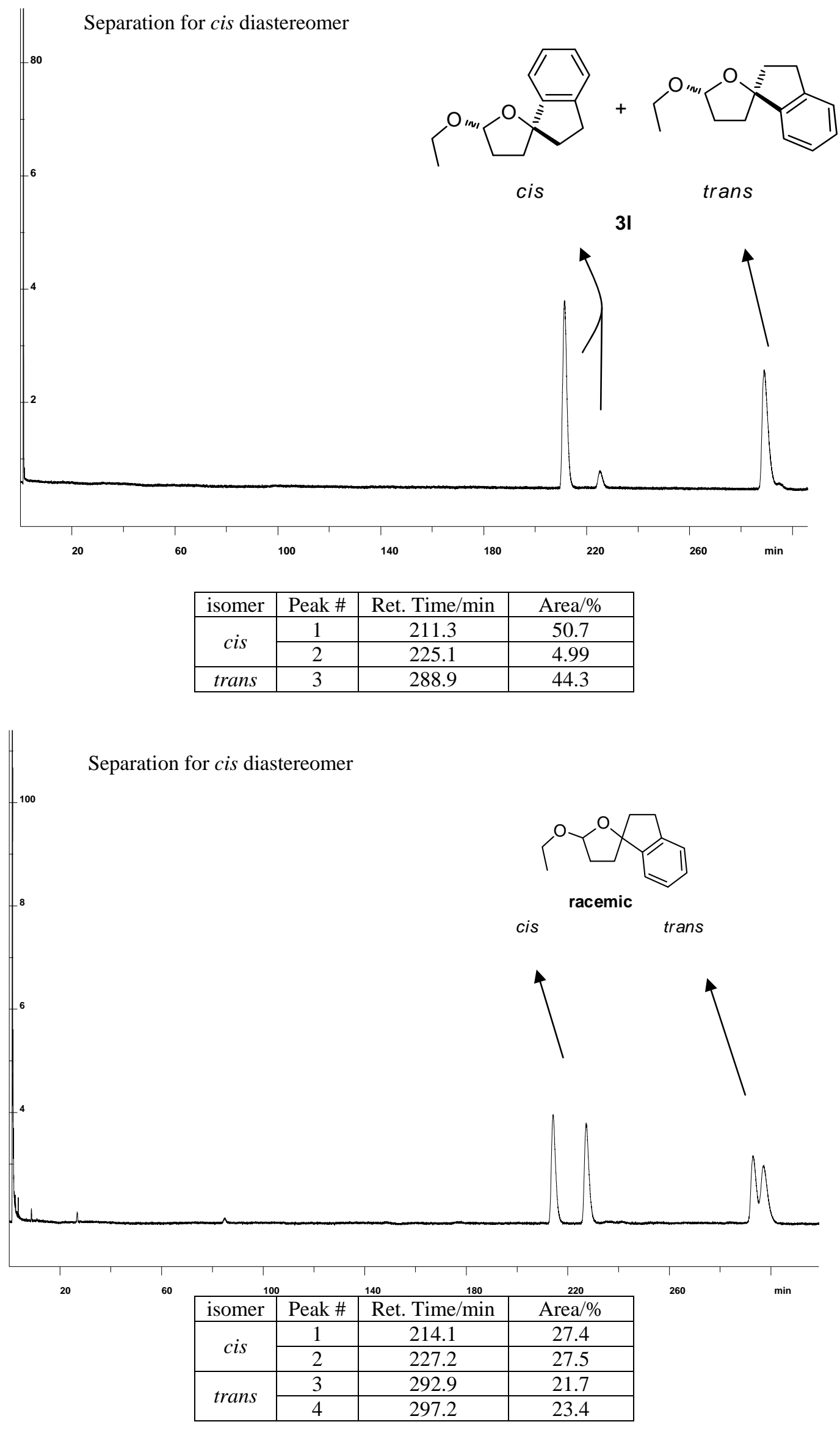

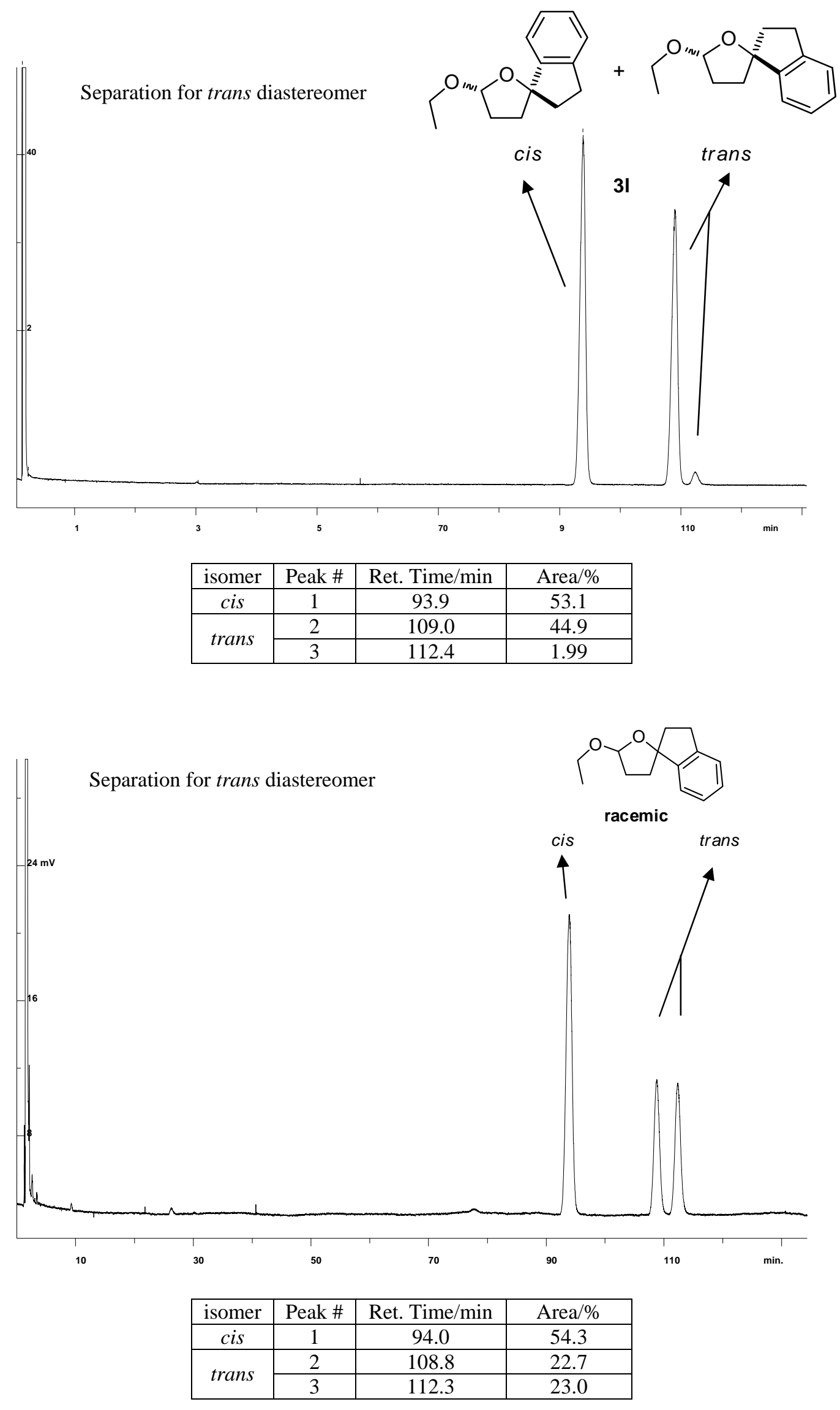

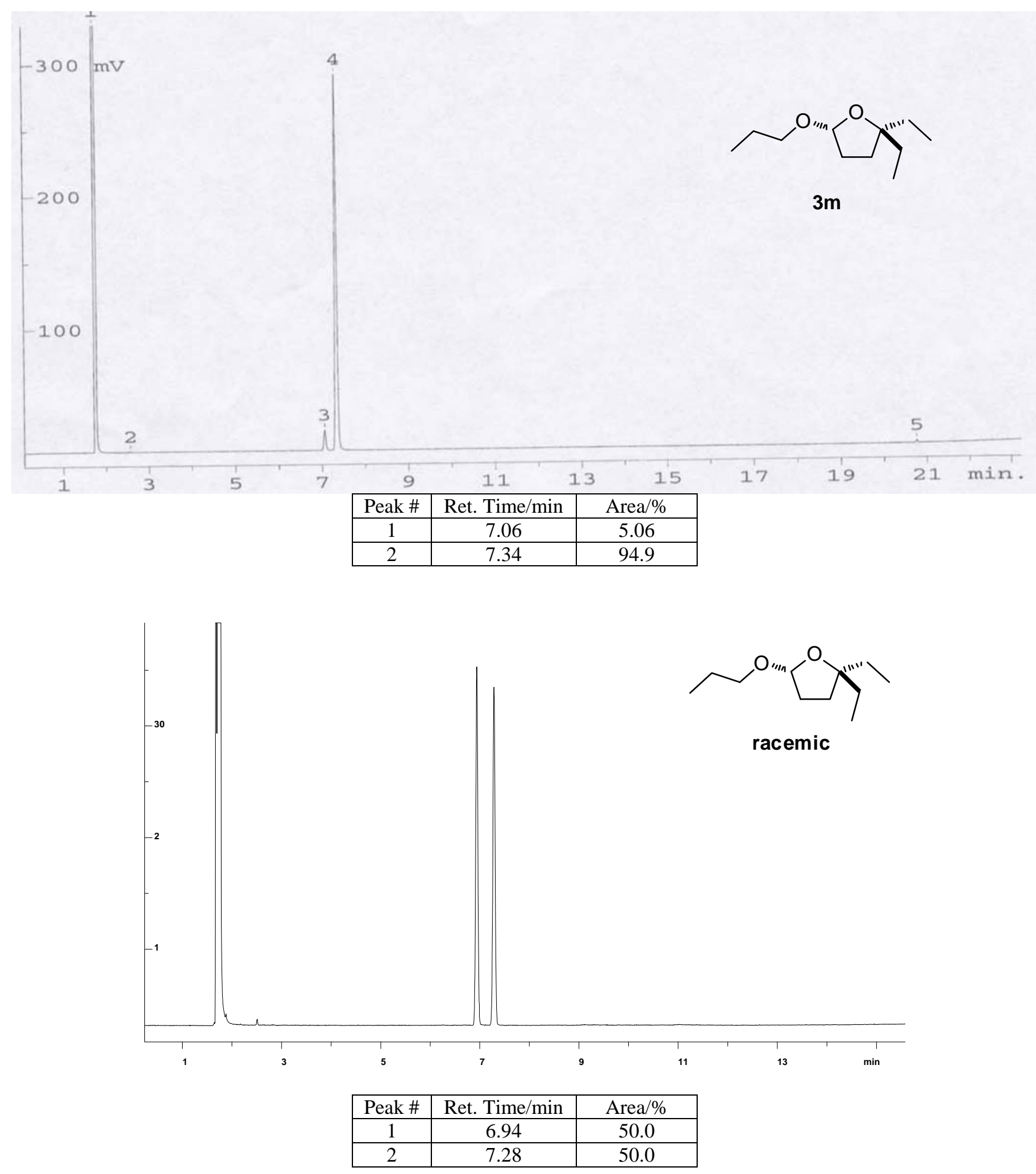
mAU

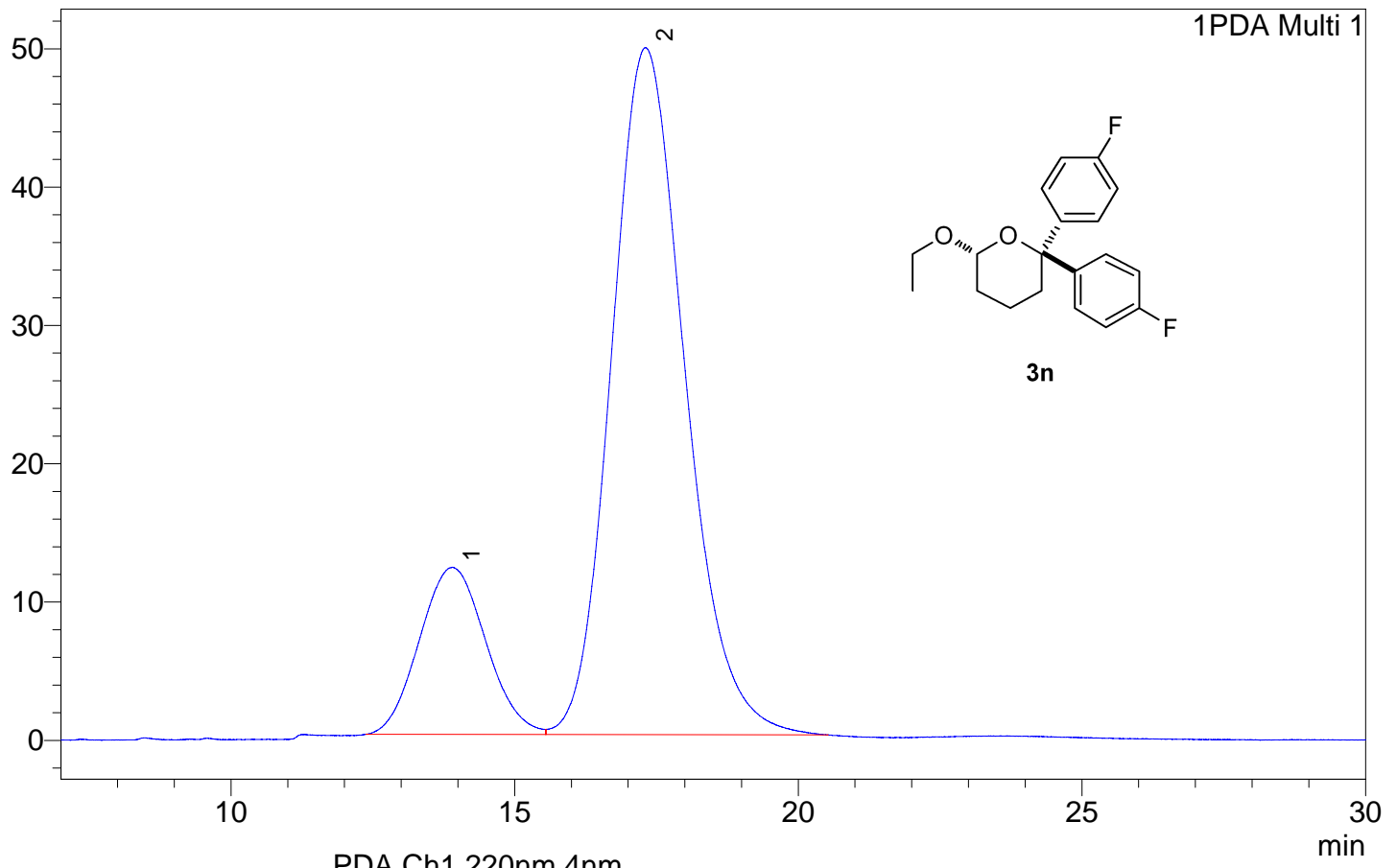

$1220 \mathrm{~nm} 4 \mathrm{~nm}$

PDA Ch1 220nm 4nm

\begin{tabular}{|c|c|c|}
\hline Peak \# & Ret. Time & Area \% \\
\hline 1 & 13.90 & 18.00 \\
\hline 2 & 17.30 & 82.00 \\
\hline Total & & 100.00 \\
\hline
\end{tabular}

mAU

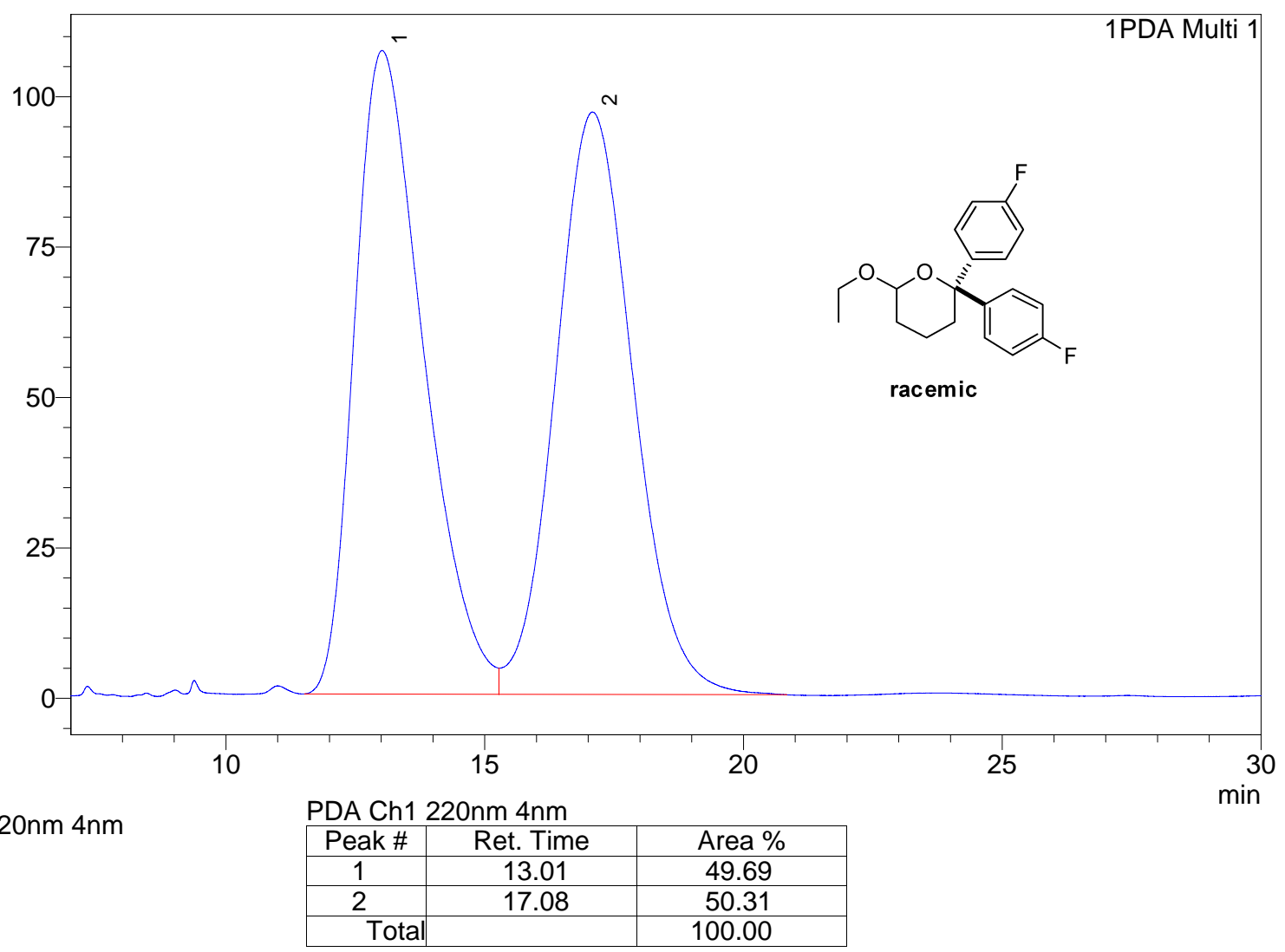




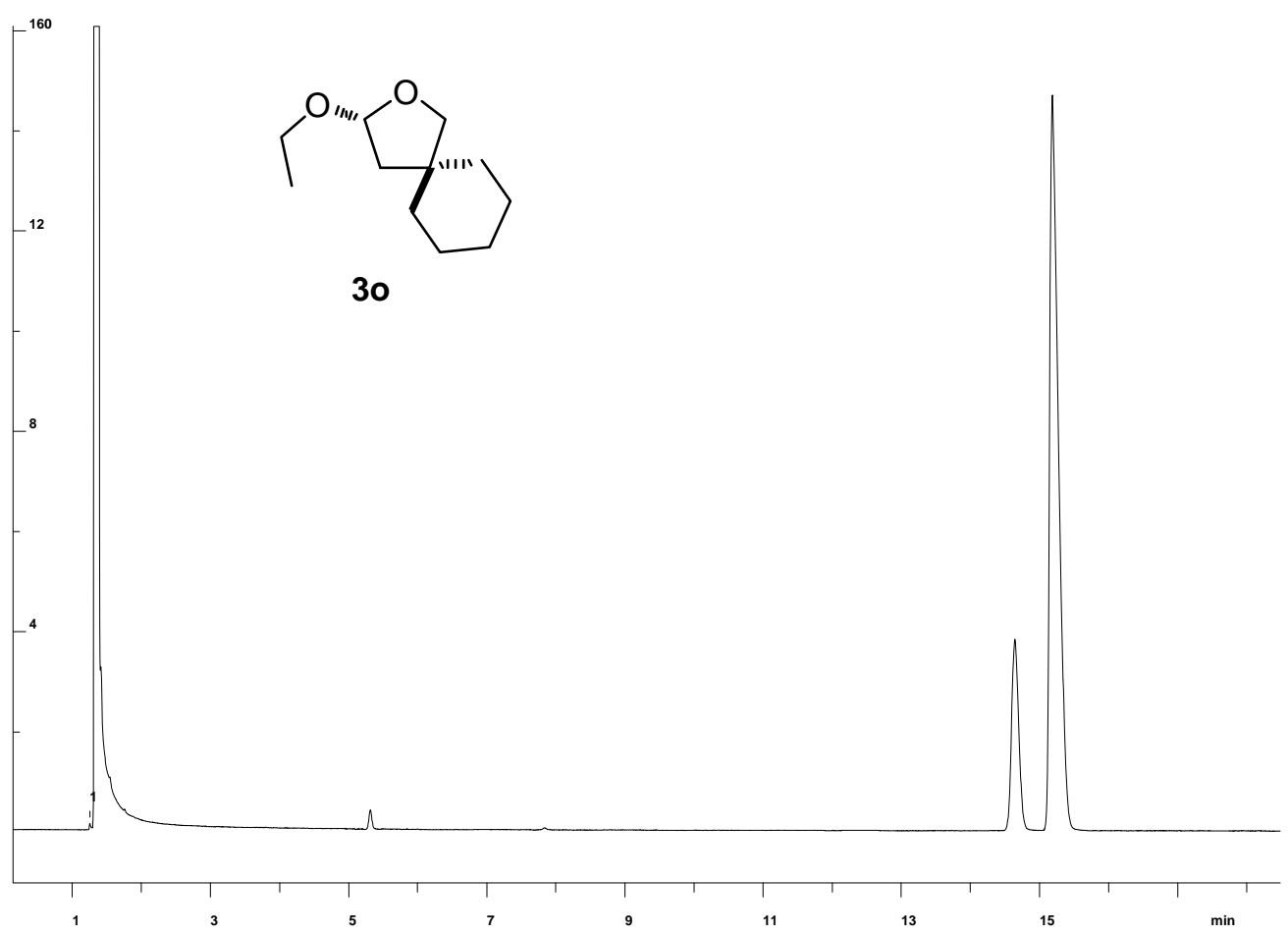

\begin{tabular}{|c|c|c|}
\hline Peak \# & Ret. Time/min & Area/\% \\
\hline 1 & 14.64 & 17.7 \\
\hline 2 & 15.19 & 82.3 \\
\hline
\end{tabular}

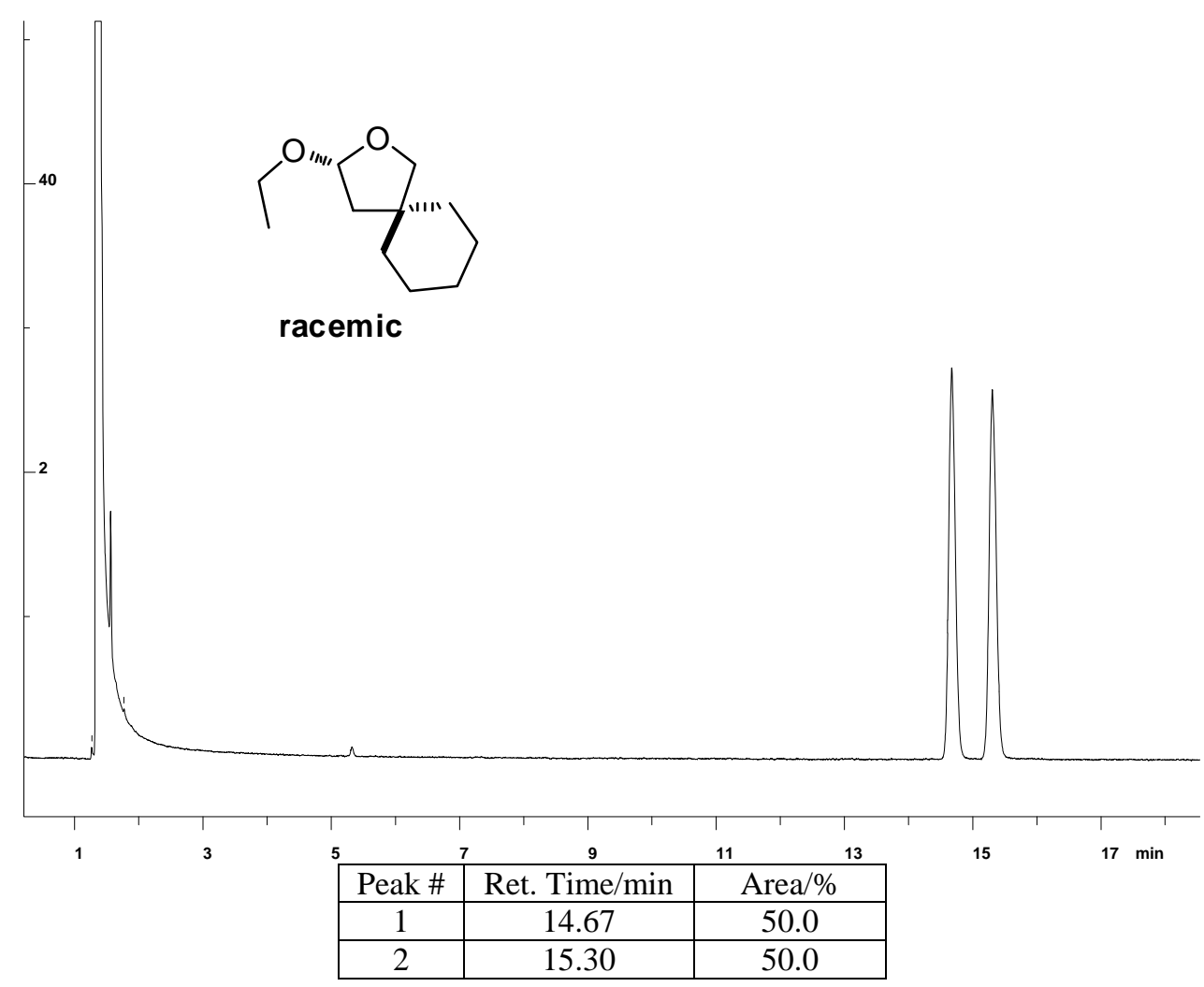



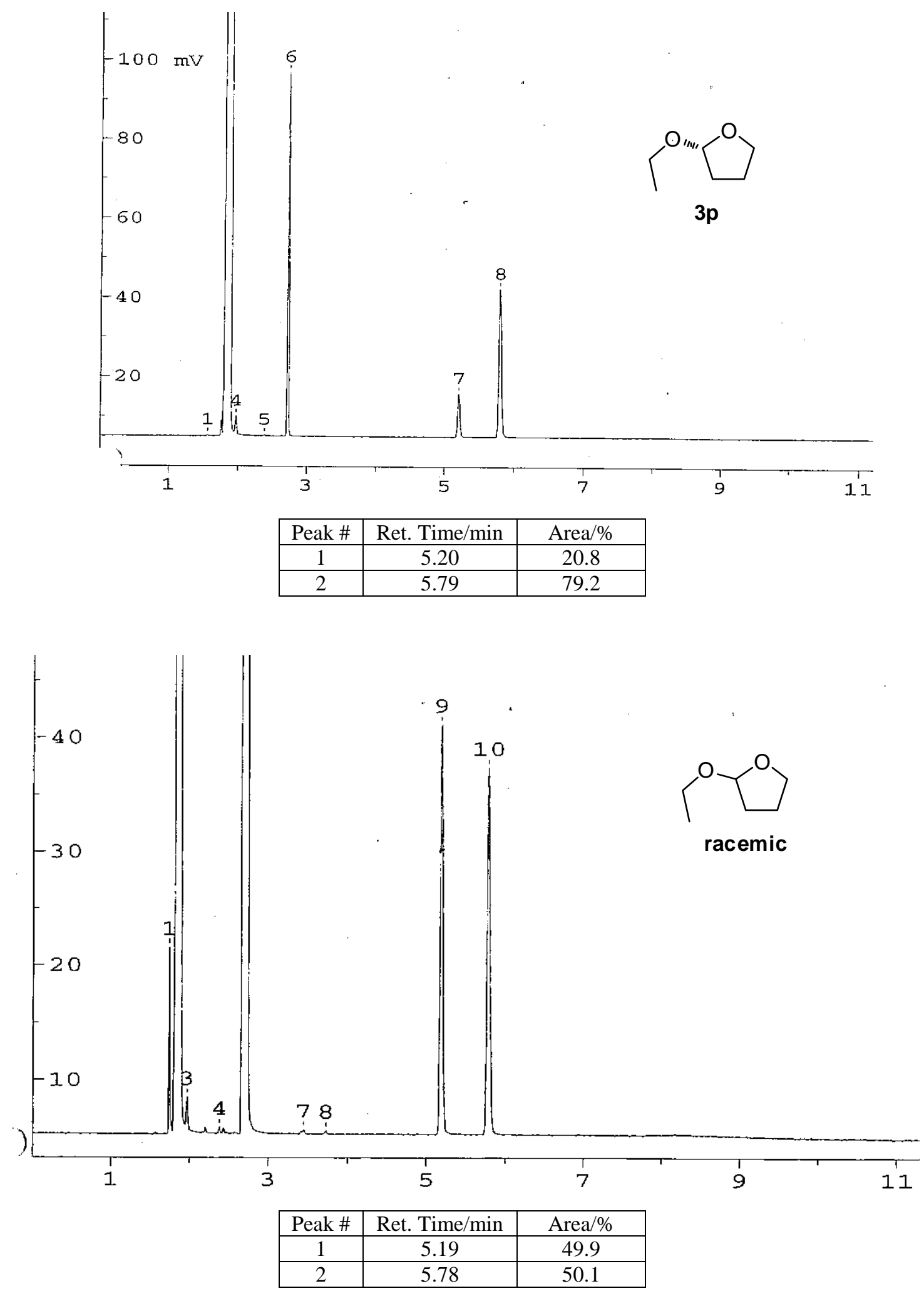